\title{
Monitoring-Cycle Based Fault Detection and Localization in Mesh All-Optical Networks
}

\author{
By \\ Hongqing Zeng, M. Sc. \\ Wuhan University
A thesis submitted to
The Faculty of Graduate Studies and Research
In Partial fulfillment of
The requirements for the degree of

\section{Doctor of Philosophy}

\author{
Ottawa-Carleton Institute for Electrical and Computer Engineering \\ Department of Systems and Computer Engineering \\ Carleton University \\ Ottawa, Ontario, Canada \\ November 5, 2006 \\ (C) Copyright \\ 2006, H. Zeng
}




$\begin{array}{ll}\begin{array}{l}\text { Library and } \\ \text { Archives Canada }\end{array} & \begin{array}{l}\text { Bibliothèque et } \\ \text { Archives Canada }\end{array} \\ \begin{array}{l}\text { Published Heritage } \\ \text { Branch }\end{array} & \begin{array}{l}\text { Direction du } \\ \text { Patrimoine de l'édition }\end{array} \\ \begin{array}{l}\text { 395 Wellington Street } \\ \text { Ottawa ON K1A ON4 }\end{array} & \begin{array}{l}\text { 395, rue Wellington } \\ \text { Ottawa ON K1A ON4 } \\ \text { Canada }\end{array}\end{array}$

Your file Votre référence ISBN: 978-0-494-27121-6 Our file Notre référence ISBN: 978-0-494-27121-6

NOTICE:

The author has granted a nonexclusive license allowing Library and Archives Canada to reproduce, publish, archive, preserve, conserve, communicate to the public by telecommunication or on the Internet, loan, distribute and sell theses worldwide, for commercial or noncommercial purposes, in microform, paper, electronic and/or any other formats.

The author retains copyright ownership and moral rights in this thesis. Neither the thesis nor substantial extracts from it may be printed or otherwise reproduced without the author's permission.
AVIS:

L'auteur a accordé une licence non exclusive permettant à la Bibliothèque et Archives Canada de reproduire, publier, archiver, sauvegarder, conserver, transmettre au public par télécommunication ou par l'Internet, prêter, distribuer et vendre des thèses partout dans le monde, à des fins commerciales ou autres, sur support microforme, papier, électronique et/ou autres formats.

L'auteur conserve la propriété du droit d'auteur et des droits moraux qui protège cette thèse. $\mathrm{Ni}$ la thèse ni des extraits substantiels de celle-ci ne doivent être imprimés ou autrement reproduits sans son autorisation.
In compliance with the Canadian

Privacy Act some supporting forms may have been removed from this thesis.

While these forms may be included in the document page count, their removal does not represent any loss of content from the thesis.
Conformément à la loi canadienne sur la protection de la vie privée, quelques formulaires secondaires ont été enlevés de cette thèse.

Bien que ces formulaires aient inclus dans la pagination, il n'y aura aucun contenu manquant.

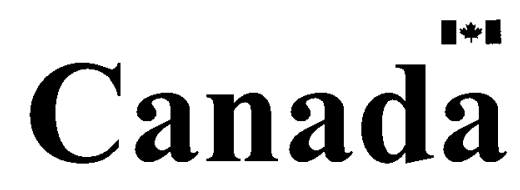




\section{Acknowledgements}

My first and deepest gratitude goes to my supervisors, Dr. Changcheng Huang and Dr. Alex Vukovic, for their guidance, patience and continuous support and encouragement throughout my graduate study and research work leading to this thesis. I gratefully acknowledge the funding support to my research work provided by Communications Reearch Centre Canada.

I would like to greatly thank my thesis readers, Dr. Wayne Grover, Dr. Trevor J. Hall, Dr. Alan Steel, Dr. Chung-Horng Lung, and Dr. John W. Chinneck, for their reviewing this thesis and insightful comments and suggestions. I would also like to thank Ms. Anna Lee and Ms. Blazenka Power for administrative helps.

Particularly I wish to thank Dr. Jing Wu for his valuable discussions, constructive suggestions, warmhearted encouragements and help advices to the research effort leading to this thesis. My sincere appreciation goes to all professors, my colleagues and friends for all courses and research related discussions, as well friendly help in all related areas: Mr. Michel Savoie, Mr. Heng Hua, Mr. Hanxi Zhang, and Mr. Scott Campbell at Communications Research Centre Canada; Prof. Oliver Yang, Dr. Qiang Chen, Dr. Yang Hong, and Ms. Haixia Yun at School of Information Technology and Engineering, University of Ottawa; Mr. Benjamin Feng, Mr. Hao Wang, Mr. Baohua Zhang, Ms. Jingmei Yang, Ms. Jie Zhu, Mr. Kamal Herb, and Mr. Yifan Zhao from Optical Network Lab, Carleton University.

Finally, my deepest gratitude goes to all my family members for their love, support, encouragement and expectation during my study. 


\section{Abstract}

The fault detection and localization (FDL) problem for mesh all-optical networks (AON) is the vital part of the survivability of such networks. This thesis proposes and verifies a novel FDL approach based on monitoring-cycles ( $m$-cycles), which are defined as cycles in an AON with the assigned network monitors and supervisory channels. An $m$-cycle based fault localization algorithm is developed together with a group of newly defined evaluation metrics: the fault localization degree, wavelength overhead, and cost reduction. Three $m$-cycle construction algorithms are developed and evaluated: the heuristic depth first searching, the shortest-path Eulerian matching, and the heuristic spanning-tree algorithms. The results obtained by applying these algorithms to some typical networks show that the $m$-cycle based FDL approach is effective and costefficient for mesh AONs.

It is also proven in this thesis that the complete fault localization is achievable if and only if an AON contains neither degree- 2 nor extended degree-2 chains. Furthermore, it is proven that the spanning-tree based $m$-cycle construction algorithm can produce an $m$ cycle set to implement the complete fault localization.

To minimize the wavelength overhead and localization degree, and to consider the limitation of lightpath length in real AONs, the $m$-cycle construction is formulated as a variant version of constrained cycle-cover problem. A novel branch-and-bound (B\&B) based two-phase algorithm is then developed to solve such problems. The analysis of the B\&B algorithm shows that feasible solutions are guaranteed and near-optimal solutions are achievable. The good performance of the algorithm is shown in simulation results obtained from typical network examples both in the real world and randomly generated.

Finally, the implementation of $m$-cycle based FDL approaches is discussed based on the simple network management protocol (SNMP), together with the transmission of alarms and notification in control channels within the control plane of an AON. 


\section{Table of Contents}

Acknowledgements .................................................................................. i

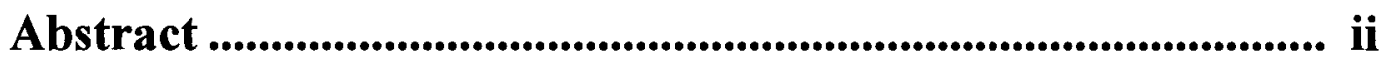

Table of Contents......................................................................... iii

List of Figures ...................................................................... vi

List of Tables........................................................................................ ix

List of Acronyms ..................................................................xiv

Chapter 1 Introduction .........................................................................1

1.1 Evolution of optical networks and fault detection/localization ........................... 2

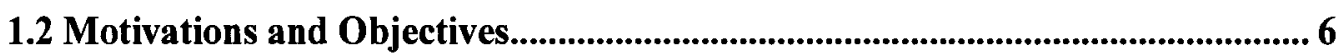

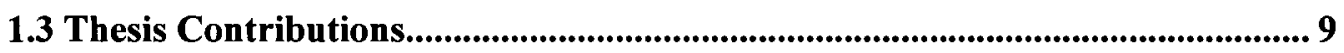

1.4 Thesis Organization ........................................................................................... 10

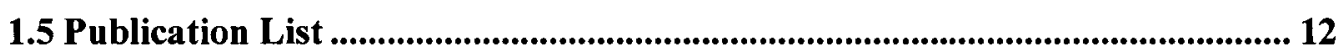

Chapter 2 Background and Literature Survey ..........................15

2.1 Network configuration and fault detection indices.................................................. 15

2.2 IP layer fault detection and localization .......................................................... 20

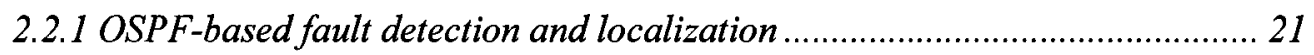

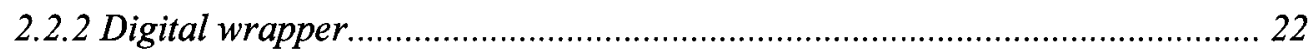

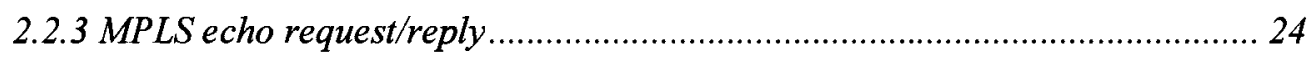

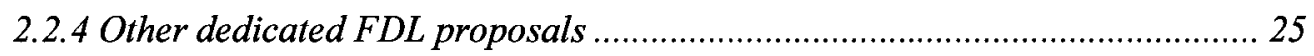

2.3 Joint-layer fault detection and localization ....................................................... 27

2.4 Optical layer fault detection and localization.......................................................... 29

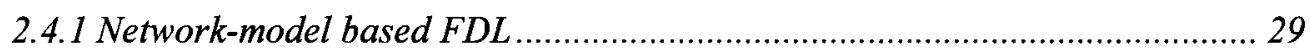

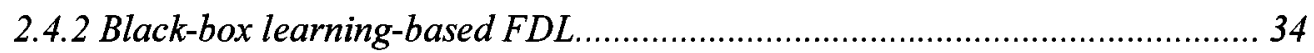

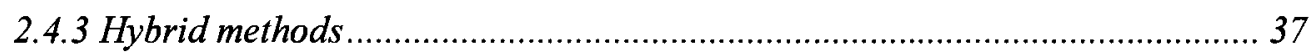

2.5 Comparison of FDL mechanisms ................................................................................ 37

Chapter 3 Monitoring-Cycle .......................................................40

3.1 Network Fault Classification .................................................................................. 40

3.2 Monitoring-Cycle ............................................................................................................. 43

3.3 M-Cycle Based Fault Localization Algorithm (MFLA) .................................. 48

iii 
3.4 A Lower Bound of M-Cycle Number for Complete Localization ..................... 52

3.5 Performance Evaluation Metrics .................................................................. 54

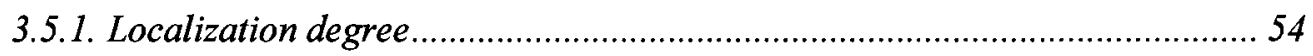

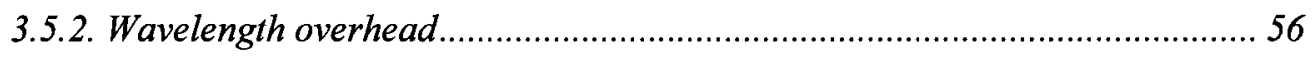

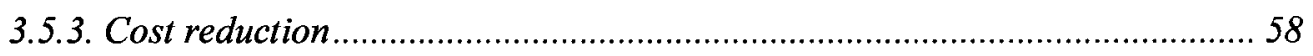

\section{Chapter $4 M$-Cycle Construction Algorithms ...............................60}

4.1 Introduction to Cycle Cover Problem (CCP)....................................................61

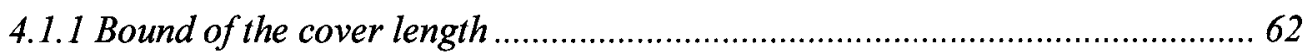

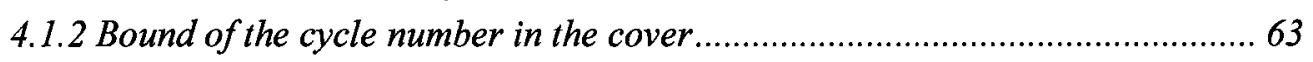

4.2 Existing Algorithms for Solving CCP .......................................................64

4.3 Heuristic Depth-First Searching (HDFS) Algorithm .................................... 70

4.4 The Shortest-Path Eulerian Matching (SPEM) Algorithm ............................... 73

4.5 Examples and Performance Evaluation ......................................................... 77

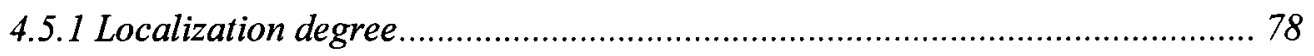

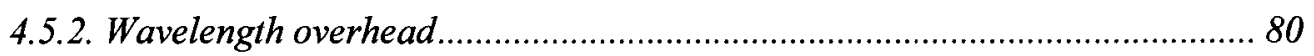

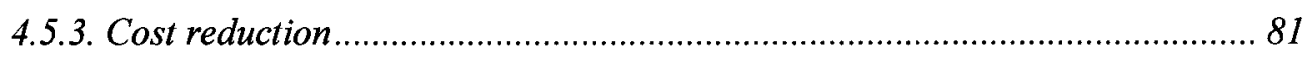

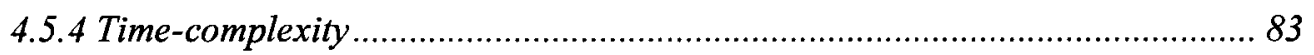

\section{Chapter 5 Condition for Achieving Complete Fault}

Localization ..........................................................................................85

5.1 Conditions for Complete Fault Localization ................................................................ 86

5.2 Heuristic Spanning-Tree (HST) based Cycle Construction Algorithm ........... 91

5.3 Complete Fault Localization and Spanning-Tree (ST) Based M-Cycle

Construction Algorithms........................................................................................... 96

5.4 Examples and Performance Evaluation .............................................................. 98

5.4.1 Localization degree .............................................................................. 98

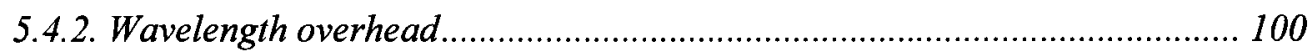

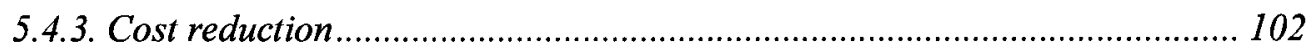

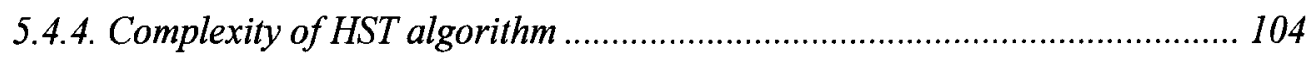

\section{Chapter 6 Full Formulation of $M$-Cycle Construction............105}

6.1 Formal definition of variant constrained cycle cover problem (vCCCP)...... 106

6.2 Solving vCCCP................................................................................................................. 108

6.2.1 Two-phase $B \& B$ algorithm ................................................................... 108

6.2.2 Link coverage, complete fault localization, and existence of feasible solution

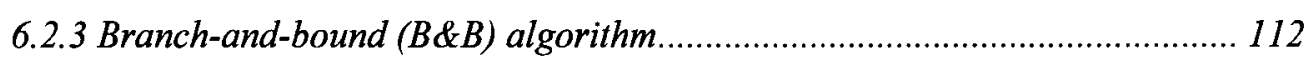


6.3 A descriptive example of solving vCCCP ............................................................ 116

6.4 Performance Analysis.................................................................................... 120

6.4.1 Localization degree, wavelength overhead, and cost reduction................... 123

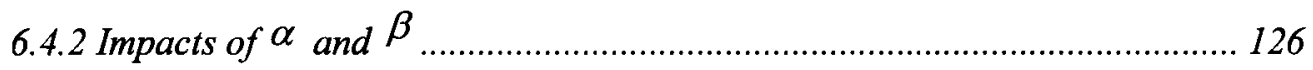

6.4.3 Complexity analysis for the $v$ CCCP solving algorithm .............................. 127

6.5 Empirical Study for vCCCP: Randomly Generated Network Examples ..... 128

Chapter 7 Implementation of MFDL .....................................130

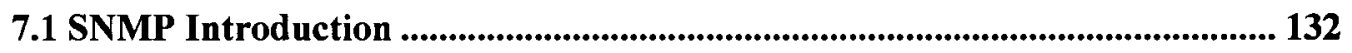

7.1.1. Structure of Management Information and MIB ...................................... 133

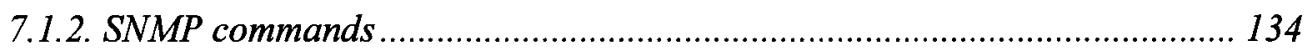

7.1.3 SNMP message types and formats ......................................................... 136

7.2 Customization of SNMP for MFDL application ................................................... 137

7.2.1 Register network alarms in the MIB tree .................................................. 138

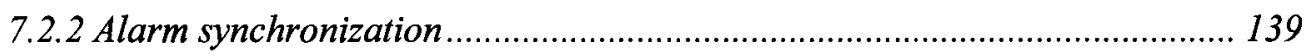

7.2.3 Faulty-Source Notification in MFDL.................................................... 140

Chapter 8 Conclusions and Future Work ...............................142

References ...........................................................................................147

Appendix A Network examples and MFLA Results with HDFS/SPEM Algorithms......................................................163

A.1 M-cycles obtained by HDFS algorithm ......................................................... 163

A.2 M-cycles obtained by SPEM algorithm ...................................................... 165

A.3 MFLA results with M-cycles obtained by HDFS algorithm ........................... 166

A.4 MFLA results with M-cycles obtained by SPEM algorithm.......................... 170

\section{Appendix B Network examples and MFLA Results with HST}

Algorithms ...........................................................................................173

B.1 M-cycles obtained by HST algorithm........................................................ 173

B.2 M-cycles obtained by HST algorithm................................................................ 175

Appendix C vCCCP Algorithm Explanation ...........................179

C.1 SPCC candidate cycles and checking-matrix for the example in Figure 6.5179

C.2 Re-number nodes in typical network examples with chain removal ............ 182

C.3 Results of vCCCP algorithm for typical network examples ............................ 184

Appendix D Empirical Study of vCCCP Algorithm................186 


\section{List of Figures}

Figure 1.1 Phases of network fault recovery process

Figure 2.1 Typical network configurations for AONs

Figure 2.2 Data plane, control plane and management plane of AONs

Figure $2.3 \mathrm{~A}$ brief pedigree of FDL approaches in AONs

Figure 2.4 The architecture and building blocks of SONET/SDH

Figure 2.5 Network-model based fault localization

Figure 3.1 A graph example and its cycle cover instance

Figure 4.1 The cycle cover obtained by HDFS for the network example in Figure 3.1

Figure 4.2 Demo of the SPEM algorithm

(a) The shortest path Eulerian matching;

(b) An Euler circuit

Figure 4.3 Eulerian circuit decompositions

Figure 4.4 Typical example networks

(a) NSFNET, 14 nodes and 21 links;

(b) ARPA2, 21 nodes and 25 links;

(c) SmallNet, 10 nodes and 22 links;

(d) Bellcore, 15 nodes and 28 links.

Figure 5.1 Example of degree-2 and extended degree-2 chains

Figure 5.2 Path existence in graph without degree-2 chain

Figure 5.3 Proof of the necessary and sufficient condition for complete fault localization

Figure 5.4 Spanning-tree and cycle cover

vi 
(a) DFST: max cover depth $=5 /$ link, average cover depth $=2.3 /$ link

(b) BFST (rooted from random nodes): max cover depth $=5 /$ link, average cover depth $=1.9 /$ link

(c) BFST (rooted from the node with maximum degree): max cover depth = 2/link, average cover depth $=1.5 /$ link

Figure 5.5 Demonstration of the HST algorithm

(a) Demonstration of the HST algorithm;

(b) The generated spanning-tree

Figure 5.6 Proof of Theorem 5.3.1

Figure 6.1 Formal definition of vCCCP

Figure 6.2 The vCCCP algorithm

Figure 6.3 Check the coverage and complete fault localization for a cycle set

Figure 6.4 The full binary tree for B\&B and an example root-leaf route

Figure 6.5 Example network $G(V, E)$ for the two-phase B\&B algorithm

Figure 6.6 The full binary tree of $B \& B$ for the network example in Figure 6.5

Figure 6.7 Network Examples for evaluating the B\&B vCCCP algorithm

Figure 6.8 Remove degree-2 chains in Bellcore

Figure 6.9 Remove degree-2 chains in NSFNET

Figure 6.10 Remove degree-2 chains in ARPA2

Figure 7.1 Architecture of the simple network management protocol (SNMP)

Figure 7.2 The hierarchical OID tree in the MIB

Figure 7.3 SNMP message formats

Figure 7.4 The enterprise sub-tree (private) in the MIB tree

vii 
Figure A.1 $M$-cycles obtained by HDFS for network examples
(a) $m$-cycles obtained by HDFS in NSFNET;
(b) $m$-cycles obtained by HDFS in ARPA2;
(c) $m$-cycles obtained by HDFS in SmallNet;
(d) $m$-cycles obtained by HDFS in Bellcore.

Figure A.2 $M$-cycles obtained by SPEM for network examples
(a) $m$-cycles obtained by SPEM in NSFNET;
(b) $m$-cycles obtained by SPEM in ARPA2;
(c) $m$-cycles obtained by SPEM in SmallNet;
(d) $m$-cycles obtained by SPEM in Bellcore.

Figure B.1 $M$-cycles obtained by HST for network examples
(a) $m$-cycles obtained by HST in NSFNET;
(b) $m$-cycles obtained by HST in ARPA2;
(c) $m$-cycles obtained by HST in SmallNet;
(d) $m$-cycles obtained by HST in Bellcore.

Figure C. 1 Re-numbered nodes in Bellcore after move degree- 2 chains

Figure C.2 Re-numbered nodes in NSFNET after move degree- 2 chains

Figure C. 3 Re-numbered nodes in ARPA2 after move degree-2 chains

viii 


\section{List of Tables}

Table 3.1. Network fault classification and detection in AONs

Table 3.2 Associative bits and codes for the network example in Figure 3.1

Table 3.3 MFLA results for the network example in Figure 3.1

Table 4.1 Bounds of CCP suboptimal solutions

Table 4.2 Comparison of MFLA results for HDFS and SPEM

Table 4.3 Comparison of wavelength overhead for HDFS and SPEMTable 4.4 Comparison of cost reduction for HDFS and SPEM

Table 5.1 Comparison of MFLA results for $m$-cycle construction algorithms

Table 5.2 Comparison of wavelength overead for $m$-cycle construction algorithms

Table 5.3 Comparison of cost reduction for $m$-cycle construction algorithms

Table 5.4 Comparison of time complexity for HDFS, SPEM, and HST algorithms

Table 6.1 The shortest paths and corresponding cycles produced by SPCC for edge in the network example in Figure 6.5

Table 6.2 Final solution of the B\&B algorithm for the example in Figure 6.5

Table 6.3 $M$-cycle construction comparison: raw results in network examples

Table $6.4 M$-cycle construction comparison: results for achieving complete fault localization in network examples

Table 6.5 Comparison of time complexity of four $m$-cycle construction algorithms

Table 6.6 Summary of MFLA results on randomly generated network examples

Table A.1 Fault localization results with $m$-cycles obtained by HDFS in NSFNET

Table A.2 Fault localization results with $m$-cycles obtained by HDFS in ARPA2 
Table A.3 Fault localization results with $m$-cycles obtained by HDFS in SmallNet

Table A.4 Fault localization results with $m$-cycles obtained by HDFS in Bellcore

Table A.5 Fault localization results with $m$-cycles obtained by SPEM in NSFNET

Table A.6 Fault localization results with $m$-cycles obtained by SPEM in ARPA2

Table A.7 Fault localization results with $m$-cycles obtained by SPEM in SmallNet

Table A.8 Fault localization results with $m$-cycles obtained by SPEM in Bellcore

Table B.1 Fault localization results with $m$-cycles obtained by HST in NSFNET

Table B.2 Fault localization results with $m$-cycles obtained by HST in ARPA2

Table B.3 Fault localization results with $m$-cycles obtained by HST in SmallNet

Table B.4 Fault localization results with $m$-cycles obtained by HST in Bellcore

Table C.1 Candidate cycles obtained by SPCC for the network example in Figure 6.5

Table C.2 The checking-matrix for verifying the existence of feasible solutions of vCCCP problem in the example network in Figure 6.5

Table C.3 Optimal solutions for SmallNet obtained by the two-phase vCCCP algorithm

Table C.4 Optimal solutions for Bellcore obtained by the two-phase vCCCP algorithm

Table C.5 Optimal solutions for NSFNET obtained by the two-phase vCCCP algorithm

Table C.6 Optimal solutions for ARPA2 obtained by the two-phase vCCCP algorithm

Table D.1 Randomly generated network example (1) with 13 nodes and 20 links

Table D.2 Optimal solutions of $m$-cycle obtained by the two-phase B\&B algorithm for randomly generated network example (1)

Table D.3 Randomly generated network example (2) with 18 nodes and 37 links

Table D.4 Optimal solutions of $m$-cycle obtained by the two-phase B\&B algorithm for randomly generated network example (2) 
Table D.5 Randomly generated network example (3) with 21 nodes and 37 links

Table D.6 Optimal solutions of $m$-cycle obtained by the two-phase B\&B algorithm for randomly generated network example (3)

Table D.7 Randomly generated network example (4) with 30 nodes and 61 links

Table D.8 Optimal solutions of $m$-cycle obtained by the two-phase B\&B algorithm for randomly generated network example (4) 


\section{List of Notations}

$\begin{array}{ll}a_{i j} & \text { The associative bit of link } e_{i} \text { for } m \text {-cycle } c_{j} \\ b_{i j} & \text { The associative bit of node } i \text { for } m \text {-cycle } c_{j} \\ \mathbf{a}_{\mathbf{i}} & \text { The associative code of link } e_{i} \\ c, c_{j} & \text { A cycle in a graph } \\ C & \text { A cycle cover for a graph } \\ \bar{d} & \text { The average node degree of a graph } \\ D & \text { The collection of all } s_{k} \\ e, e_{i} & \text { A link of a graph } \\ E & \text { Edge set of a graph } \\ F_{i} & \text { The faulty candidate flag of link } e_{i} \text { for a given alarm code } \\ G, G(V, E) & \text { A graph with the vertex set } V \text { and edge set } E \\ G_{n} & \text { Cost reduction } \\ G_{n}^{\prime} & \text { Revised cost reduction with fair comparison } \\ I & \text { A spanning-tree } \\ K_{2} & \text { The localization degree } \\ L & \text { The complete graph of a single edge and its two endpoints } \\ M & \text { The number of links in a graph } \\ m_{j} & \text { The number of elements in an } m \text {-cycle set for a graph } \\ \mathbf{m}, \mathbf{m}_{\mathbf{k}} & \begin{array}{l}\text { The status indication bit for an } m \text {-cycle } c_{j} \\ N\end{array}\end{array}$

xii 


$\begin{array}{ll}V & \text { Vertex set of a graph } \\ w_{i}^{j} & \text { The } j \text {-th component of a cycle associative vector } \\ \mathbf{w}_{\mathbf{i}} & \text { A cycle associative vector } \\ W_{\text {O }} & \text { Average wavelength overhead } \\ W O H_{m a x} & \text { Maximum wavelength overhead } \\ x, y & \text { Network nodes } \\ Z & \text { The limitation in hops for a lightpath length } \\ \alpha & \text { The cost of a network monitor } \\ \beta_{i} & \text { The cost of a wavelength for link } i \\ \Lambda_{a v g} & \text { Average number of reserved wavelength for } m \text {-cycles in a link } \\ A_{m a x} & \text { Maximum number of reserved wavelength for } m \text {-cycles in a link } \\ \Delta & \text { The number of total available wavelengths in a link } \\ \bullet \bullet & \text { Set cardinality, or the cycle length if "•" is a cycle } \\ <\bullet, \bullet> & \text { The inner product of two vectors } \\ \oplus & \text { Exclusive OR operation }\end{array}$




\section{List of Acronyms}

\begin{tabular}{ll} 
ANN & Artificial Neural Network \\
AON & All-Optical Network \\
ASN.1 & Abstract Syntax Notation One \\
ATM & Asynchronous Transfer Mode \\
B\&B & Branch-and-Bound \\
BER & Bit Error Ratio \\
BIP & Bit-Interleaved Parity \\
CCP & Cycle-Cover Problem \\
CCCP & Constrained Cycle-Cover Problem \\
CDCC & Cycle Double Cover Conjecture \\
CPP & Chinese Postman Problem \\
DeMUX & De-Multiplexer \\
DFS & Depth-First Searching \\
DWDM & Dense Wavelength-Division Multiplexing \\
EDFA & Erbium-Doped Fibre Amplifier \\
FDDI & Fibre Distributed Data Interface \\
FDL & Fault Detection and Localization \\
FEC & Forward Error Correction \\
FSM & Finite State Machine \\
HDFS & Heuristic Depth-First Searching \\
HOS & High Order Statistical \\
\hline
\end{tabular}

xiv 
IETF Internet Engineering Task Force

IMP Integer Mathematical Programming, Integer Programming

IP Internet Protocol

IS-IS Intermediate System-to-Intermediate System routing protocol

LAN Local Area Network

LOL Loss of Light

LOS Loss of Signal

LSA Link State Advertisement

LSP Label Switching Path

LSS Loss of Sequence Synchronization

$M$-cycle $\quad$ Monitoring-Cycle

MCCP Minimum Cycle-Cover Problem

MEMS Micro-Electro-Mechanical Systems

MFDL $\quad M$-cycle based Fault Detection and Localization

MFLA $\quad M$-cycle based Fault Localization Algorithm

MIB Management Information Base

MPLS Multi-Protocol Label Switching

MUX Multiplexer

NMS Network Management System

NMU Network Management Unit

NOC Network Operation Center

O-QoS Optical Quality of Service 


\begin{tabular}{|c|c|}
\hline OADM & Optical Add/Drop Multiplexer \\
\hline OAM\&P & Operations, Administration, Maintenance, and Provisioning \\
\hline $\mathrm{OC}$ & Optical Carrier \\
\hline OEO & Opto-Electro-Opto \\
\hline OID & Object Identifier \\
\hline OPM & Optical Power Meter \\
\hline OSA & Optical Spectrum Analyzer \\
\hline OSNR & Optical Signal-to-Noise Ratio \\
\hline OTDR & Optical Time-Domain Reflectometer \\
\hline OTN & Optical Transport Networks \\
\hline OXC & Optical Cross-Connect \\
\hline $\mathrm{P} 2 \mathrm{P}$ & Point-to-Point \\
\hline PDU & Protocol Data Unit \\
\hline PT & Pilot Tone \\
\hline QoS & Quality of Service \\
\hline RBSP & Rolling Back Signaling Protocol \\
\hline REI & Remote Error Indication \\
\hline RFC & Request for Comments \\
\hline RWA & Routing and Wavelength Assignment \\
\hline SDH & Synchronous Digital Hierarchy \\
\hline SMI & Structure of Management Information \\
\hline SONET & Synchronous Optical Network \\
\hline SPCC & Shortest-Path Candidate Cycle Construction \\
\hline
\end{tabular}

xvi 
SPE Synchronous Payload Envelop

SPEM Shortest-Path Eulerian Matching

SNMP Simple Network Management Protocol

STS Synchronous Transport Signals

TCP Transmission Control Protocol

UDP User Datagram Protocol

WAN Wide Area Network

xvii 


\section{Chapter 1}

\section{Introduction}

N all-optical network (AON) is a communications network that works completely
in the optical domain. The user data in AONs are carried by and kept in optical signals along the transmission paths (usually called lightpaths or optical channels). The optical signals are only converted into electrical domain at the communication sources and destinations. Nowadays, the tremendous bandwidth demands of growing InternetProtocol (IP) data traffic and novel services have been pushing the conventional communications networks to the physical limitations in the electrical domain. Therefore AONs become more attractive and viable for future telecommunications and data networks because of the avoidance of optical-electro-optical (OEO) conversion and thus the removal of physical transmission limitation in electrical domain. The AONs can provide ever-increasing channel data rate, channel number and channel density via the massive deployment of dense wavelength-division multiplexing (DWDM) technologies [1-2]. However, a very short disruption of service caused by a network fault may lead to a very high data loss in such networks. Numerous schemes have been proposed to improve the survivability for AONs since the first architecture of optical network was proposed [3-7]. In most survivable mechanisms, it is simply assumed that the information of network faults has been obtained before invoking those schemes. So the fault detection

$$
-1-
$$


and localization (FDL) is the prerequisite for and of critical important to survivable optical networks.

Fault detection and localization (FDL) in general networks is not a new problem and have been intensively studied for years in various areas, e.g., power distribution systems, electrical circuits, industrial control systems, and communication networks [8]. In optical networks, the FDL techniques have been developed along with the evolution of network architectures and technologies.

\subsection{Evolution of optical networks and fault}

\section{detection/localization}

Optical network technology became a reality in 1980's and led to the wide use of optical networks in local and wide areas (LAN/WAN), such as fibre distributed data interface (FDDI), fibre channel, and synchronous optical network/synchronous digital hierarchy (SONET/SDH) [9-10]. The beaconing process and digital wrapper of data packets were the mainstream techniques of fault detection and localization for optical ring networks (e.g. FDDI) and SONET/SDH, respectively [11-13]. During the beaconing process, each station in the ring yields to an incoming beacon frame and repeats it. Stations at downstream of the fault domain in the ring generates beacon frames which are being repeated by all stations up to the fault domain. Since the beacon frames contain the location information of the beaconing station, the location of the faulty source is apparent 
to a manager located at any of the stations. The optical network technology in these early networks played only a role of transmission pipe. The booming advances and developments in optical technologies in 1990's, e.g. micro-electro-mechanical systems (MEMS), optical add/drop multiplexer (OADM), Erbium-doped fibre amplifier (EDFA) and Raman amplifier, in addition to the advances in fibre technology, etc., have driven those primitive optical networks to be more flexible and efficient. Based on these new technologies, various novel optical network architectures were proposed, which could support more flexible topologies, provide new services and be more operationally efficient [14-15]. At this stage, numerous FDL approaches for conventional networks were applied to such optical networks [16]. Proposals based on probabilistic and deterministic fault-propagation models [17-21], finite state machine [22-23], neural network and artificial intelligence [24], and expert systems [25] have been studied. These proposals were implemented in either centralized or distributed styles.

Due to the electronic bottleneck in conventional networks and optical networks with opto-electro-opto (OEO) conversion, as well as the cost-efficiency of managing bits in all-optical networks (AONs), AON architectures emerged in the middle of 1990's and received extensive study since that time [15]. Today, AONs with the deployment of DWDM technology have been prevalent in long haul and backbone communication networks [26-27]. However, existing FDL approaches for conventional networks cannot be directly applied to AONs due to the lack of electrical terminations, or the unaffordable price and complexity of implementation [28]. For example, expert systems for FDL are based on the previous experience of network faults stored in databases. But it is widely believed that AONs will eventually support dynamic lightpath provisioning, which makes

\section{$-3-$}


a discrepancy between the history data in those expert systems and the current status of the networks, and thus leads to inaccurate FDL results. For the same reason, fault propagation models (either probabilistic or deterministic) have to be updated frequently. Even if such updates could be done quickly enough to trace the dynamic lightpath provisioning, it would put a hefty load on the network management unit and consequently delay the FDL process. Even some methods deployed in optical networks with optoelectro-opto (OEO) conversion cannot be transplanted to AONs, like the examples in $[28]$.

To handle such particular issues, mechanisms of FDL for AONs have been developing in both physical layer and IP layer, or both. Most routing protocols in the IP layer, e.g. Open Shortest Path First (OSPF) routing protocol [104] and IS-IS intra-domain routing protocol [105], have inherent functionality of FDL [29]. Some dedicated protocols were also proposed for FDL in the IP layer, such as the MPLS echo request/reply protocol [30] and an end-to-end keep-alive protocol [31]. Some joint optical and IP layer methods were proposed to accelerate the detection speed [32-34]. Unfortunately, the long detection time in the IP layer (typically at a second-level or subsecond-level, even with some accelerating techniques) makes it difficult to achieve time-critical recovery in AONs. Therefore, some effective and efficient FDL mechanisms at the optical layer are needed for AONs. A fault detection scheme was developed in [35], which assigned monitors to sinks of each optical multiplex section and optical transmission section. Another scheme proposed in [23] modeled all possible states of each link as a finite state machine (FSM). The FSM for every link tracked the current state of the link by assigning a monitor to the link. Clearly, all potential faults could be 
completely detected and located by this monitor-per-link approach. However, it is not always feasible to implement the monitor-per-link scheme in large-scale networks because of the large number of expensive optical network monitors (e.g. transceivers) required for monitoring. Besides the cost of monitoring devices themselves, the operation, administration and maintenance expense cannot yet be neglected. Other than assigning a monitor per link, some authors placed a monitor to each established lightpath [37]. Some heuristics were proposed to reduce the required number of monitors based on the information of redundant alarms. This scheme was effective when it was proposed, since the number of lightpaths in an AON at that time was relatively small and they did not change frequently once established. However, the number of lightpaths has soared so much nowadays with the deployment of DWDM technology that this scheme will introduce a huge cost due to the large number of required monitors. Furthermore, the dynamic lightpath provisioning will make the monitor-per-path approach more difficult if it is not completely infeasible. Actually, it is not necessary to put monitors on all links, lightpaths, or nodes, for detecting and locating network faults. For example, some authors proposed a diagnosis method with sparse monitoring nodes (multiple monitors may be required within one node) specifically for crosstalk attacks, which could be considered as special cases of network faults in wide sense [38-39].

This thesis will focus on the optical layer FDL approaches with fast fault detection time, accurate fault localization results, and efficient overall costs. Currently $50 \mathrm{~ms}$ is the industrial standard time constraint for fault recovery. Therefore, FDL schemes could complete within this time constraint are considered as fast FDL. 


\subsection{Motivations and Objectives}

The importance of FDL in AONs has been recognized in the research community since the emergence of AONs. However, the reported proposals of FDL are not as abundant as the counterparts in other research areas for AONs, such as protection and restoration mechanisms, due to the following challenges for FDL in AONs.

The first challenge of the AON FDL is the strict time constraint. The fault recovery process consists of five phases, as shown in Figure 1: fault detection, localization, notification, traffic recovery (protection or restoration), and the optional phase, reversion (normalization) [40-41]. In industry standards, the acceptable time constraint for fault recovery in optical networks, is usually within 50 milliseconds. With the consideration of leaving enough time for other phases, especially the time-consuming fault notification [42], such a time-constraint makes the AON FDL difficult even at the physical layer. Thus some FDL approaches at physical layer without complex online calculation are expected.

The second challenge is the high cost of monitoring devices. Currently monitors are assigned either per link or per path in most AON FDL proposals. A huge number, usually unacceptable for real applications, of monitors are required even with some optimization for the monitor assignment for FDLs in AONs. Therefore some novel monitor assignment algorithms are needed to significantly reduce the number of required monitors.

$$
-6-
$$




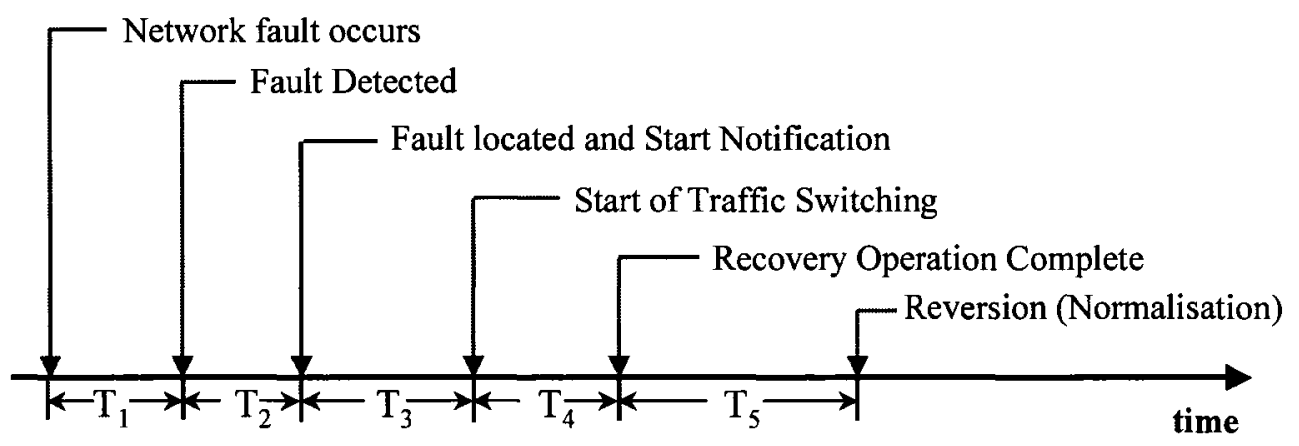

Figure 1.1 Phases of network fault recovery process

1) $T_{1}$ - Fault detection; 2) $T_{2}$ - Fault localization; 3) $T_{3}$ - Fault notification;

4) $T_{4}$ - Traffic recovery; 5) $T_{5}$ - Traffic reversion

Finally, but no less important, the dimension of AONs are being increased continuously. Furthermore, it is widely believed that AONs will eventually support dynamic lightpath provisioning. Therefore, new AON FDL approaches must be scalable and support dynamic lightpath provisioning.

To meet such challenges, the fundamental research work is the study of the monitorper-link approaches, e.g., the FSM-based FDL schemes. However, the dramatic high expense of the required monitoring devices extremely impedes their deployment. In this thesis, a novel FDL approach is developed at the physical layer for AONs, which is fast, cost-efficient, scalable and insensitive to dynamic lightpath provisioning. Fundamentally, this approach is based on the fact that the topology of an AON can be decomposed into a set of cycles, in which each node and link of the AON appears at least once. Network faults are detectable in such cycles and the potential faulty sources can be found in the common part of those cycles with fault alarms. In such mechanisms, only one monitoring device is assigned to each cycle. Since the number of cycles is much less than the number of links, the number of required monitoring devices can be significantly reduced. The

$$
-7-
$$


payment for the savings of monitoring devices is that a link might appear more than once in the cycle cover and therefore leads to additional wavelength cost. Nevertheless, the cost of monitoring devices is usually more than that of wavelengths, and we can minimize the cover depth for links in the cycle cover construction to keep the cost of wavelengths to minimum. With a given cycle cover, the cover depth for a link is defined as the number of cycles in the cover in which the link appears. More importantly, such cycle-based FDL approaches can pre-calculate the candidate faulty source for alarms before the real time network operation. Consequently the main calculation load of the FDL can be removed and thus it dramatically speeds up the FDL process. Such approaches are especially applicable to backbone network carrier providers, who have sufficient spare wavelengths but need extra investment for monitoring devices. Specifically, to develop the cycle-based FDL for AONs, the following objectives are posted below for this thesis work.

- Show the feasibility and develop the framework of an FDL approach to achieve accurate and fast FDL, and significantly save the cost of AON monitoring devices at the same time. The new approach decomposes AONs into cycles, which is defined as the monitoring-cycles ( $m$-cycles);

- Develop an $m$-cycle based fault localization algorithm (MFLA) with the minimum online calculation;

- Define a set of metrics for evaluating the performance of the $m$-cycle based FDL approach;

- Develop some $m$-cycle construction algorithms and compare their performance in terms of the newly defined metrics; 
- Check the conditions for achieving the complete fault localization via $m$-cycle based FDL approaches. The complete fault localization is defined as the case that the fault localization algorithm produces only a single faulty candidate for each network fault (for single failure scenarios);

- Find effective and efficient $m$-cycle construction algorithms to produce an $m$-cycle set for achieving the complete fault localization results, if the given AON satisfies the conditions for complete fault localization.

\subsection{Thesis Contributions}

The main contributions of this thesis are as the following (in the order of their significance),

- The development of the $m$-cycle based FDL approach for mesh AONs, including the proof of the feasibility for such approaches and the novel definition of the $m$ cycle;

- The definition of necessary and sufficient conditions for achieving the complete fault localization. The complete fault localization is defined as meaning that the faulty source can be localized to a single network element for every network fault;

- The model of a variant constrained cycle-cover problem (vCCCP) for $m$-cycle construction with the consideration of cycle number, cover cost, and cycle length limit for AONs that satisfy the complete FDL conditions;

- The development and performance analyses of the branch-and-bound based $m$ - 
cycle construction algorithm for solving the above vCCCP.

- The development of an $m$-cycle based fault localization algorithm (MFLA);

- The verification of that HST $m$-cycle construction algorithm produces a set of $m$ cycles to achieve complete fault localization results if the given AON satisfies certain conditions;

- The improvement of three $m$-cycle construction algorithms: the heuristic depthfirst searching (HDFS), the shortest-path Eulerian matching (SPEM), and the heuristic spanning-tree (HST) based algorithms, as well as the comparison of their performance;

- The definition of a set of metrics for evaluating the performance of $m$-cycle based FDL approaches;

- The derivation of a loose lower-bound for the number of $m$-cycles to achieve the complete fault localization results;

\subsection{Thesis Organization}

The remain part of this thesis is organized as the following,

Chapter 2 describes the background knowledge of FDL, typical AON architectures and network configurations, as well as the review of existing AON FDL schemes in the recent literature.

Chapter 3 introduces the concept of $m$-cycle and analytically proves the feasibility of $m$-cycle based FDL for mesh AONs. An $m$-cycle based fault localization algorithm 
(MFLA) is also developed in this chapter, followed by the definition of a set of metrics for the performance evaluation. In the last part of this chapter a lower-bound of the number of $m$-cycles is given for achieving the complete fault localization results.

Chapter 4 firstly intoduces the cycle cover problem (CCP) and gives a brief survey of existing algorithms for solving CCP. Then it develops two $m$-cycle construction algorithms: HDFS and SPEM. These algorithms are applied to four typical network examples: NSFNET, ARPA2, SmallNet, and Bellcore. Their performance is compared in terms of the evaluation metrics introduced in Chapter 3 , based on the results obtained from those example networks.

Chapter 5 defines the extension of a basic concept in graph theory, degree- 2 chain, and proves that the complete fault localization results are achievable in an AON if and only if its topology contains neither a degree- 2 chain nor an extended degree- 2 chain. It also describes the heuristic spanning-tree based $m$-cycle construction algorithm (HST) and proves that the HST algorithm produces a set of $m$-cycles to implement the complete fault localization, if an AON satisfies such a condition.

Chapter 6 formulates the $m$-cycle construction to a variant cycle-cover problem ( $\mathrm{vCCCP}$ ) with the consideration of minimizing the cycle number, cover cost, and cycle length limit. A two-phase branch-and-bound (B\&B) based algorithm is then proposed for solving the vCCCP. The algorithm is applied to four typical network examples used in Chapter 4 and other four randomly generated network examples. The results are analyzed and compared with previous algorithms: HDFS, SPEM, and HST.

Chapter 7 discusses the implementation of $m$-cycle based FDL approaches in AONs. The SNMP is described and customized for sending alarms using the TRAP message 
from monitoring devices in $m$-cycles to the network management system, and delivering faulty-source information to network nodes using the SET message.

Chapter 8 concludes the thesis and lists some potential directions for future research on this topic.

\subsection{Publication List}

The following is a list of the candidate's publications during his Ph.D. study so far. Publications $[1,2,5,6,7,11]$ are on the $m$-cycle based MFDL developed in this thesis. Publications $[3,5,8]$ report an MFDL schemes using an improved end-to-end keep-alive protocol. Publication [10] develops a pilot-tone based MFDL for OXC port-mapping errors. Publications $[4,9,12,13,15]$ are on the model and optimization for AON testbed design.

[1] Hongqing Zeng, Changcheng Huang, and Alex, Vukovic, "Achieving complete fault localization based on monitoring-cycles in mesh all-optical networks," submitted to Photonic Network Communications

[2] Hongqing Zeng, Changcheng Huang, and Alex Vukovic, “A Novel Fault Detection and Localization Scheme for Mesh All-Optical Networks Based on MonitoringCycles," Photonic Network Communications, Vol. 11, No. 3, May 2006

[3] Hongqing Zeng, Alex Vukovic, and Changcheng Huang, "Fast Fault Detection and Localization in WDM Networks," SPIE NewsRoom (Technical Paper), Jan. 2006 [Online Journal] http://newsroom.spie.org/x861.xml

[4] Alex Vukovic, Hongqing Zeng, Michel Savoie, and Heng Hua, "Modeling and Optimization of Physical Layer Parameters for Development of Research Metro 
Networks," Photons, Vol. 3, No. 1, Pages 19-21, Spring 2005

[5] Hongqing Zeng, Alex Vukovic, and Changcheng Huang, "A novel end-to-end fault detection and localization protocol for wavelength-routed WDM networks," in Proceedings of SPIE Photonics North 2005 (SPIE PN'05), Toronto, Ontario, Canada, Sept. 12-14, 2005

[6] Hongqing Zeng, Changcheng Huang, and Alex Vukovic, "Spanning-Tree Based Monitoring-Cycle Construction for Fault Detection and Localization in Meshed All-Optical Networks," in Proceedings of IEEE international Conference on Communications (IEEE ICC'05), Seoul, Korea, May 16-20, 2005

[7] Hongqing Zeng, Changcheng Huang, Alex Vukovic and Michel Savoie, "Fault Detection and Path Performance Monitoring in Meshed All-Optical Networks," in Proceedings of IEEE Global Telecommunications Conference 2004 (IEEE GLOBECOM'04), Dallas, TX, USA, 29 Nov.-3 Dec. 2004

[8] Hongqing Zeng, Alex Vukovic, Changcheng Huang, and Michel J. Savoie, "Achieving Fast Fault Recovery in All-Optical Networks," in Proceedings of IEEE/OSA OFC/NFOEC'05, Anaheim, CA, USA, Mar. 6-11, 2005

[9] Hongqing Zeng, Alex Vukovic, Heng Hua, J. Michel Savoie and Changcheng Huang, "Transport Performance of an All-Optical Metro WDM Network Based on Dynamic All-Optical Switches," in Proceedings of IEEE/OSA OFC/NFOEC'05, Anaheim, CA, USA, Mar. 6-11, 2005

[10] Hongqing Zeng, Alex Vukovic, Michel Savoie and Changcheng Huang, "Wavelength-Routing Fault Detection in an AON Testbed Utilizing Concatenated Pilot Tones," in Proceedings of SPIE Photonics North 2004 (SPIE PN'04), Vol. 5579, Pages 620-627, Ottawa, Ontario, Canada, Sept. 27-29, 2004

[11] Hongqing Zeng, Changcheng Huang, and Alex Vukovic, "Monitoring Cycles for Fault Detection in Meshed All-Optical Networks," in Proceedings of 2004 International Conference on Parallel Processing Workshops (IEEE ICPP'04), Pages 434-439, Montreal, Quebec, Canada, Aug. 15-18, 2004

[12] Hongqing Zeng, Alex Vukovic, Heng Hua, J. Michel Savoie, Changcheng Huang, 
and Thao Nguyen, "Optimization of Physical Layer Parameters for an All-Optical Network Testbed," in Proceedings of the 4th IASTED International Conference on Wireless and Optical Communications (WOC'04), Pages 703-708, Banff, Alberta, Canada, July 8-10, 2004

[13] Hongqing Zeng, Alex Vukovic, J. Michel Savoie, and Changcheng Huang, "Optimisation of All-Optical Network Testbed Regarding NRZ and RZ Modulation," in Proceedings of the 17th IEEE Canadian Conference on Electrical and Computer Engineering 2004 (IEEE CCECE'04), Vol. 1, Pages 428-432, Niagara Falls, ON, Canada, May 2-5, 2004

[14] Zhao Ma, Xiaoming Lin, and Hongqing Zeng, "A Multiresolution reconstructive Algorithm Based on Network Theory for Electrical Capacitance Tomography," in Proceedings of the 17th IEEE Canadian Conference on Electrical and Computer Engineering 2004 (IEEE CCECE'04), Vol. 1, Pages 107-110, Niagara Falls, ON, Canada, May 2-5, 2004

[15] Hongqing Zeng, Alex Vukovic, Heng Hua, J. Michel Savoie, and Changcheng Huang, "Optimisation of All-Optical Network Testbed," in Proceedings of the 3rd IASTED International Conference on Wireless and Optical Communications (WOC'03), Pages 75-79, Banff, Alberta, Canada, July 14-16, 2003 


\section{Chapter 2}

\section{Background and Literature Survey}

\subsection{Network configuration and fault detection indices}

typical AON usually consists of key building blocks such as optical cross-
connect (OXC), multiplexer (MUX), de-multiplexer (DeMUX), transmitter, receiver, optical amplifier, etc [43-44]. Figure 2.1 shows the typical network node configurations for AONs. With the technology advancement, the data rate in an optical channel has soared from $2.5 \mathrm{Gbps}$ to $10 \mathrm{Gbps}$ and even to $40 \mathrm{Gbps}$. Meanwhile, with the deployment of DWDM technology, the number of optical channels multiplexed into a single fiber reached 432 even in 2001 [57]. This number is still being raised in the research community. Therefore, AONs promise the significant increase of the network bandwidth. On the other hand, the Internet Protocol (IP) has been dominant as the de facto standard in data communication networks. There is now a growing consensus that IP-over-WDM networks create the optimized transport network. A number of architectures have been proposed for IP-over-WDM networks in the past years [114-115]. In the IP-centric AON architectures, the functionalities and services of the network are grouped into three planes: data plane, control plane, and management plane, as shown in Figure 2.2. 


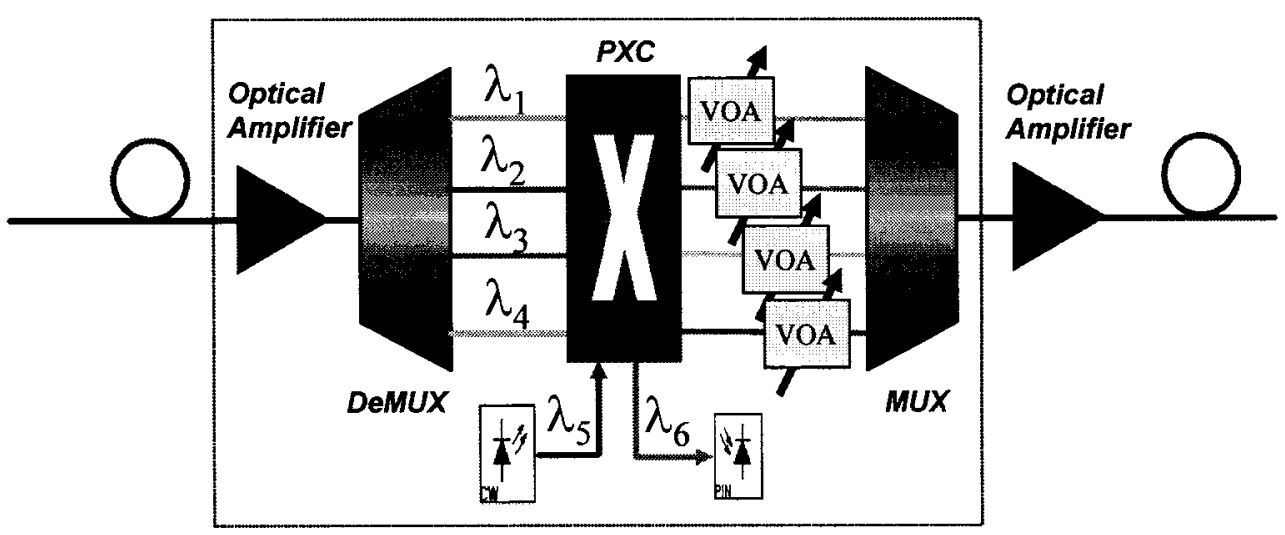

Figure 2.1 Typical network node configuration for AONs

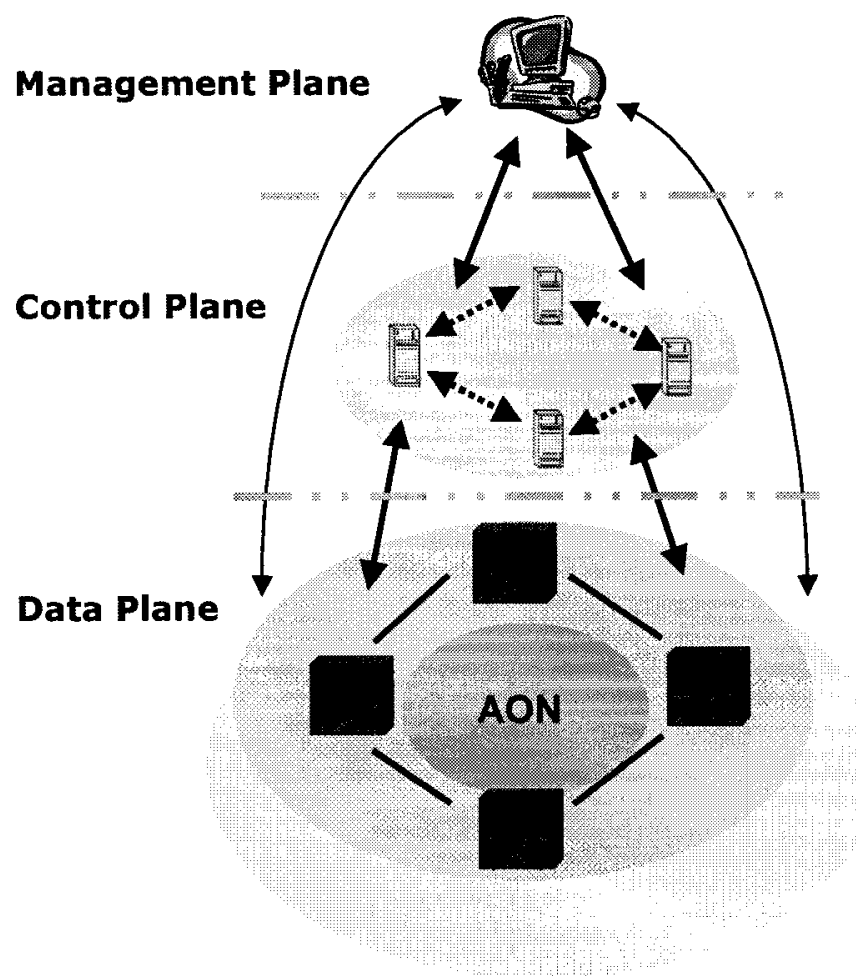

Figure 2.2 Data plane, control plane and management plane of AONs 
The data plane typically provides the function of user data transmission and includes necessary protocols and signaling for that purpose. The control plane is positioned between the data and management planes. It consists of architectures and protocols that evolve equipment configuration in data plane, dynamic network resources provisioning and service activation, traffic engineering, QoS support, etc. The management plane has ultimate control over all data plane and control plane entities. It provides the function related to network operations, administration, maintenance and provisioning (OAM\&P).

The work in this thesis focuses on the FDL in data plane and assumes that the control plane and management plane are reliable. Although the survivability and FDL in control plane and management plane are also important, it is out of the scope of this thesis. Interested readers please refer to [116-119].

It has been shown that in AONs the impairments of optical signals due to the transportation limits, e.g., chromatic dispersion, laser chirp, crosstalk, non-linearity, etc., can be minimized by applying application-optimized optical layer network elements [4547], and therefore the risk of network faults is reduced. However, some network faults are still inevitable due to any one of the hardware failure, software failure, human mistakes of network operators and maintainers, and so on [48-49]. A network fault refers to the event that potentially interrupts the network service, including signal failure (hard failure) and signal degradation (soft failure). It might be also referred as network failure, fault, defect, etc. All network faults can be also considered as the results of the degradation of the following performance indices that exceed the tolerable thresholds,

- Optical channel power;

- The central wavelength of an optical channel (frequency drift); 
- Optical signal-to-noise ratio (OSNR);

- Optical spectrum;

- Bit error ratio (BER), etc.

At the physical layer, network faults can be detected by measuring the above network performance indices in AONs [31,50]. The simplest and most cost-efficient performance monitor is the optical power meter (OPM). It measures the received optical power at any location in an AON and compares to an expected value. However, small but detectable changes in optical power may not necessarily affect the communications. Additionally, the observation of optical power does not provide the information of the quality of signals such as the OSNR, signal wavelength, BER, etc. To obtain the more detailed diagnosis, the optical spectrum analyzer (OSA) is usually deployed in AONs. OSAs can detect the power shift among the wavelengths within one channel even if the shift does not change the total power over all channels. However, the long detection time and high cost impede more wide use of OSAs. Pilot tones (PTs) are signals modulated on and distinguishable from the communication signals, which have much smaller amplitude and different frequencies compared to the main signals. Pilot tones can be used either as identifiers for tracking optical channels [51-52], or for monitoring signal impairments such as the chromatic dispersion, non-linearity, etc [53-54]. The Optical time domain reflectometer (OTDR) sends a high power laser light pulse down the fiber and looks for return signals from backscattered light in the fibre itself or reflected light from connector or splice interfaces. OTDRs are generally applied to detecting bends and losses in the fibre. More comprehensively, the fundamental measurement of the overall network performance is 
the BER [55-56]. Any other measurement only gives the result about the "signal quality", which could be "high quality" (e.g., perfect optical power and central wavelength of an optical channel) but the signal would be still useless to end-users (e.g., due to bit flips). Nevertheless, those indices still provide some measurements of optical transmission performance and they are especially suitable for cost-sensitive applications. Some methods directly measuring the BER, e.g. eye-diagram sampling, can be also considered for fault detection in optical networks. Many Forward Error Correction (FEC) coders can also generate a BER signal for performance monitoring at the IP layer. Interested readers may refer to [133].

A single or combination of multiple indices can be applied to network fault detection. In AONs, either in the single index or in the multiple indices case, the FDL can be conducted in either the IP or the physical layer, or the joint layer of both. In the IP layer, most routing protocols, such as OSPF [104] and IS-IS [105], can detect the up/down state of any node or link in a network, thus node or link faults that put impacts on the IP layer are detectable for such routing protocols. Digital wrapper is another widely used FDL scheme at IP layer, in which fault detection and localization is achieved by overhead inspection inserted in data packets or special probing packets. Recently, some dedicated FDL protocols have been developed, e.g. the MPLS echo request/reply protocol and GMPLS Link Management Protocol (LMP). Joint-layer (IP and physical) techniques were also developed in some literature to accelerate the FDL speed, e.g., a token based joint two-layer FDL scheme is proposed in [74]. In the physical layer, the FDL approaches can be classified into two categories: a) the network-model based approaches, 
and b) the black box learning-based approaches. The network-model based approaches include finite state machine models, probabilistic failure models, and deterministic network models. The black-box based approaches mainly include expert systems, casebased systems, artificial neural networks, and proactive learning systems. Some hybrid methods were also reported. A brief pedigree is shown in Figure 2.3 to summarize the above approaches.

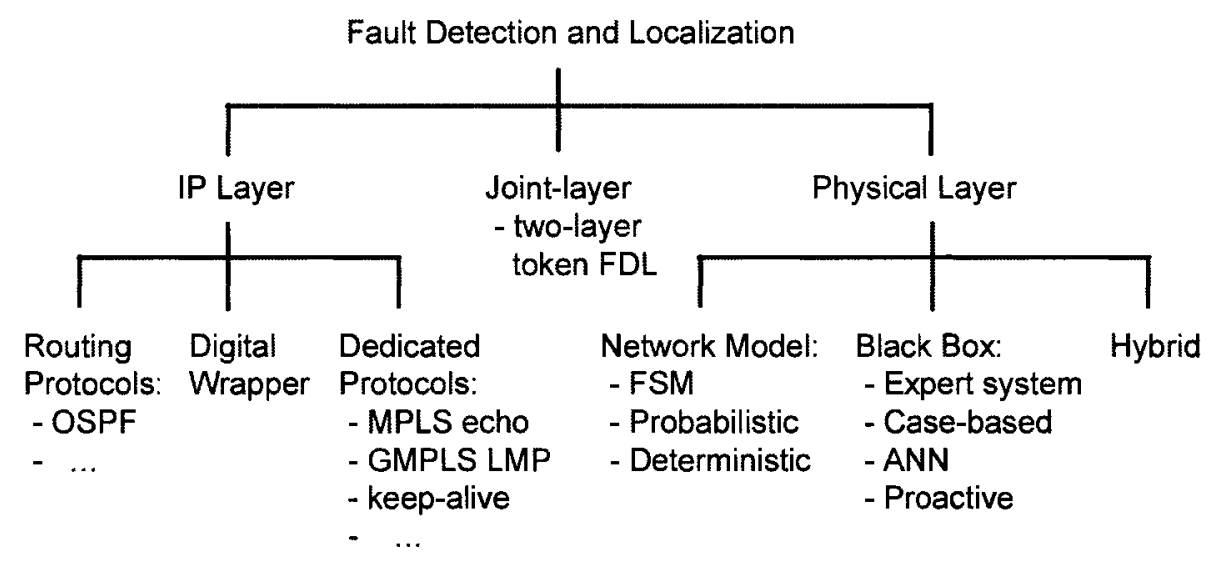

Figure 2.3 A brief pedigree of FDL approaches in AONs

\subsection{IP layer fault detection and localization}

Signaling protocols in the IP layer for fault detection and localization, including OSPF, digital wrapper, MPLS echo request/reply, and other dedicated proposals, are surveyed in this sub-section. 


\subsubsection{OSPF-based fault detection and localization}

Fault detection was first integrated into the second version of OSPF in 1998 [104]. Each router in an OSPF network keeps the information of the network topology and the associated weights. The shortest paths to various destinations are calculated on the basis of such information. To achieve fault detection, two adjacent routers periodically exchange the so-called "Hello" messages to maintain their connections. The time period between two consecutive Hello messages is defined as the "HelloInterval" (typically 10 seconds). The router assumes that a network fault occurs if it does not receive any Hello message from a neighbor in a given time period, called "RouterDeadInterval" (typically 40 seconds or 4 HelloIntervals). Once a network fault is detected, the router generates a new link state advertisement (LSA) message and flood throughout the network. Affected routers then update the network topology information and re-calculate the shortest paths in their routing tables. Clearly, the fault detection time depends on the HelloInterval and RouterDeadInterval [106]. The fault detection time might be reduced by setting a smaller value to the RouterDeadInterval. But a small RouterDeadInterval may cause a false fault alarm due to the congestion related loss of Hello messages (not the real network fault caused loss of Hello messages). Another substantial way to reduce the fault detection time is to decrease the HelloInterval. However, it pushes heavier overhead to the network, increases the chance of network congestion, and increases the chance of loss of hello messages and consequently, false alarms.

With typical RouterDeadInterval and HelloInterval values, the fault detection time can take anywhere between 30 and 40 seconds. The authors of [29] quantitatively 
analyzed the impacts of RouterDeadInterval and HelloInterval values on fault detection time. They accelerated the OSPF fault detection to 2-6 seconds, while the grade of acceleration depends on various network topologies and network load, by optimizing those values through simulations. Although the Hello protocol is practical, cost-effective, and already integrated into the existing OSPF routing protocol, its speed is far below the time requirement for FDL in AONs. Furthermore, the OSPF Hello method, or any other method in this layer, would also not necessarily localize an optical layer fault because the IP layer logical link topology (which is all OSPFs see) is generally quite different than the underlying physical layer topology. And one physical layer span cut can map into a large number of apparently simultaneous logical link failures in the OSPF layer.

\subsubsection{Digital wrapper}

Digital wrapper, a data-packet overhead inspection technique, is widely used for fault detection in SONET/SDH optical networks. The layered architecture and building blocks of SONET/SDH are shown in Figure 2.4. For the purpose of network operations, administration, maintenance, and provisioning (OAM\&P), enough information is inserted into the overhead of each layer, e.g., section overhead, line overhead, etc.

The synchronous transport signal (STS) and optical carrier (OC) levels are electrically equivalently defined in SONET for the fiber-optic based transmission hierarchy. Various STS (OC) levels provide different line rates, e.g., STS-1 (OC-1) for 51.840 Mbps, STS-3 (OC-3) 155.520 Mbps, etc. The section overhead contains 9 bytes of the transport overhead accessed, generated, and processed by section-terminating equipment. One byte in the overhead (named B1) is the section bit-interleaved parity 
code (BIP-8) - this is an even parity used to check for transmission errors over a regenerator section. Its value is calculated over all bits of the previous STS-N frame then placed in the B1 byte of STS-1 before scrambling.

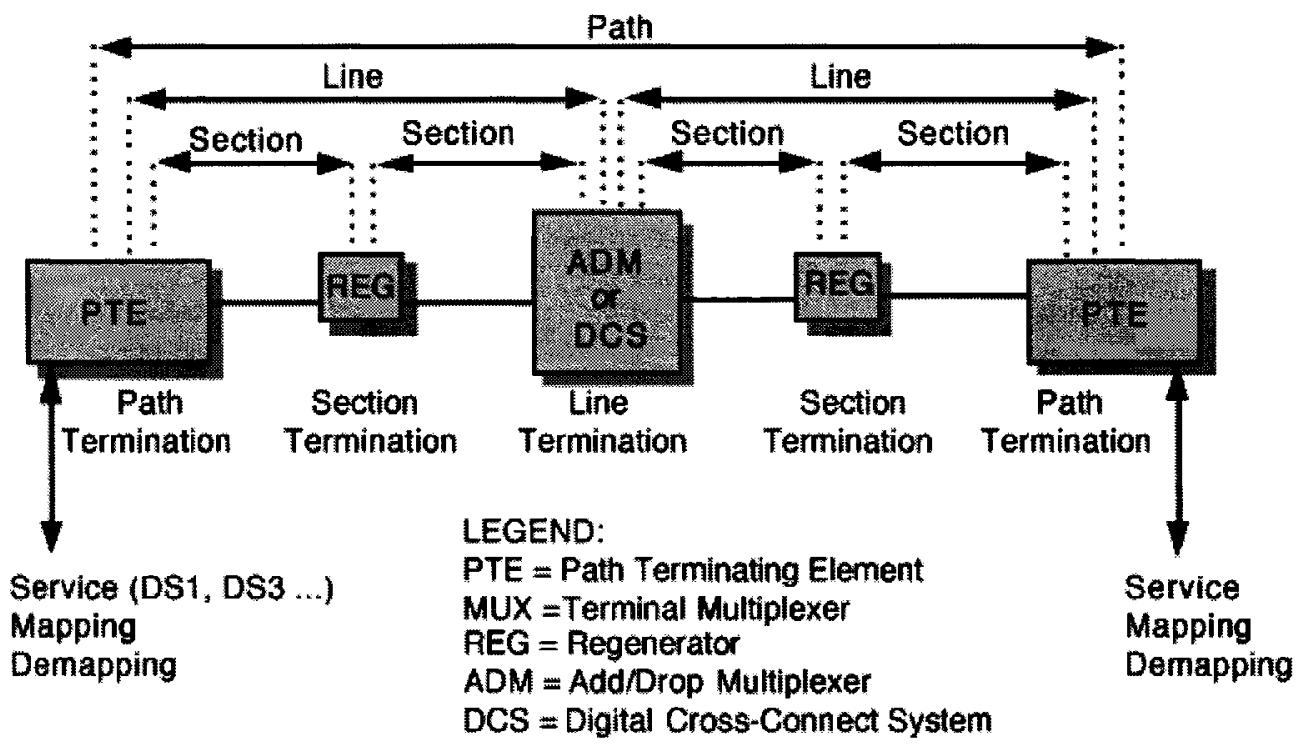

Figure 2.4 The architecture and building blocks of SONET/SDH

Line overhead contains 18 bytes of overhead accessed, generated, and processed by line-terminating equipment. A byte of BIP- 8 codes is found in the line overhead (named B2) - this parity code byte is used to determine if a transmission error has occurred over a line. Similar to B1, it is even parity and is calculated over all bits of the line overhead and STS-1 synchronous payload envelop (SPE) of the previous STS-1 frame before scrambling. The value is placed in the B2 byte of the line overhead before scrambling.

Based on the calculation of $\mathrm{B} 1 / \mathrm{B} 2$, together with other fault detection related bytes in the overheads, SONET defines a group of alarms for network anomalies, defects, and 
failures, e.g., B1 error, B2 error, loss of sequence synchronization (LSS), remote error indication (REI), etc. Even the bit error ratio (BER) of the transmission connection can be estimated through $\mathrm{B} 1$ and $\mathrm{B} 2$, which may enable the proactive network fault detection. The details of the hierarchical architecture, packet overhead, and definitions of alarms for OTN can be found in [60].

The technique of digital wrapper, together with the forward error correction (FEC) coding [133], has been adopted in optical transport networks (OTN). The digital wrapper makes use of management bits, symbols, frames, packets, or cells wrapped around user data. The digital wrapper is effective and easy to implement. Examples are implemented and standardized in SONET/SDH [60]. However, such techniques also inevitably incur a processing and cost penalty.

\subsubsection{MPLS echo request/reply}

Multiprotocol label switching (MPLS) is a versatile solution to address the problems faced by present-day networks — speed, scalability, quality-of-service (QoS) management, and traffic engineering. MPLS gives network operators a great deal of flexibility to divert and route traffic around link failures, congestion, and bottlenecks. As MPLS becomes more and more popular at the IP layer, it integrates a simple and efficient technique to detect data plane failures in a point-to-point (P2P) MPLS Label Switched Path (LSP). This technique relies on the information carried in an MPLS "echo request" and "echo reply", and mechanisms for transporting the echo reply [30]. The MPLS echo request/reply mechanism was proposed to IETF in March 2002 [108] and has been improved, finally leading to the IETF RFC 4379 [109]. 
In MPLS echo request/reply mechanism, on an established data path, the source node sends a request message to the destination through the control plane. Once the destination receives the request, it returns back the echo reply message to the source but the reply message goes exactly following the data path. At each intermediate node, if there is a network fault along the data path and the reply message cannot go further to upstream, a fault notification will be distributed to all upstream nodes via control plane. Here the control plane is assumed to be reliable. An echo request is a UDP packet and contains FEC coding information. When an MPLS echo request is received, the receiver is expected to verify that the control plane and data plane are both healthy and in synchronism. An MPLS echo reply is a UDP packet containing return code and subcode. Detailed packet formats of echo and reply, as well as return code and subcode, are defined in [109].

\subsubsection{Other dedicated FDL proposals}

Besides the existing techniques described above, more and more dedicated FDL protocols are proposed for optical networks while they are becoming prevalent in modern telecommunication networks.

Island-based fault localization and restoration architecture was proposed in [110]. In this method, the network is decomposed into overlapping sub-networks called islands. Each node of the network belongs to an island, which consists of the node itself (called a center node), all links incident to it, all adjacent nodes, and extra nodes and links necessary to reroute traffic when any component of the island fails. Within an island, each adjacent node is a detector node and perceives link failures between itself and the 
center node. Such failure detection is typically fulfilled via a loss of signal alarm. According to the criteria for choosing extra nodes and links for rerouting, islands fall into three categories: minimal island, shortest-path island, and 2-stage island. This method simplifies the fault detection (in an island-by-island fashion), but a network fault cannot be further localized within the island. This may significantly decrease the utilization of network resources, especially when the fault occurs within a large island.

Another proposal deploys the similar technique in OSPF but maintains hello packets between the source and destination node pair for an end-to-end lightpath, rather than the adjacent node pairs $[31,111]$. The source node of each lightpath keeps sending hello packets to the destination node exactly following the path for data traffic. The destination node generates an alarm once a certain number of consecutive hello packets are missed within a given time period. Then the network management unit (NMU) collects all alarms and locates the faulty source based on the network topology, and sends fault notification messages via the control plane to either the source node or to all upstream nodes along the lightpath. This protocol detects faults in the data plane by keeping hello packets following the same path as the data packets, while it significantly reduces the overall fault recovery time by reducing the fault notification time (notification executed in the control plane). The reported performance evaluation shows that it can achieve fast fault detection, and at the same time, the overhead brought to the user data by hello packets is low [111].

Some researchers proposed a broadcasting fault detection protocol [112]. In this protocol the destination at a lightpath backwardly broadcasts a small control packet, called detecting signal, to all nodes along the path. The detecting signal traverses through 
the control plane that is assumed to be reliable. Each recipient of the detecting signal is triggered to send a small acknowledgement signal back to the destination through the lightpath. At the destination, any missed acknowledgement implies anomalies along the lightpath and thus a network fault is detectable. Either a timer or a counter is set at the destination to monitor the detecting-acknowledgement signals. Since the arrival of an acknowledgement guarantees an healthy link, the faulty source along the lightpath can be located to the node one more hop further than the most upstream node with successful acknowledgements. An improved version of broadcasting FDL, called rolling back signaling protocol (RBSP), was reported in [113]. In RBSP the detecting signal is sent to the closest upstream node from the destination of an established lightpath. The recipient checks the data line and if there is no fault it passes (rolls back) the detecting signal to its upstream neighbor. This procedure repeats till the detecting signal arrives in the source node of the lightpath. In this way RBSP suppresses the broadcasting thus improves the network resources utilization, but at the payment of longer fault detection time.

\subsection{Joint-layer fault detection and localization}

In AONs even a single fiber cut will affect multiple lightpaths due to the high channel density, which implies extremely high load of routing re-calculation for traffic recovery and thus hampers the fast traffic recovery against network faults. To accelerate the FDL speed of IP-layer protocols, a hybrid distributed fault-management protocol was proposed for single fiber failure [32]. This protocol combines Link State Protocol to disseminate 
and update the information of only physical connectivity, with a distributed localinformation based signaling algorithm for connection and fault management. The fault detection relies on the end nodes of the faulty link, e.g., the detection of loss of signal. When a link failure occurs, the link state protocol is triggered to disseminate information about this topological change (only the physical connectivity) to all nodes so that future connections will bypass the faulty part of the network. The link state protocol is only triggered when failure occurs to reduce the recovery action. Meanwhile, each node maintains the information database to keep the correlation between lightpaths and physical connections. When a node detects a network fault, it finds affected resources by looking up the information database and generates a failure notification message to be sent to all affected resources.

Recently, a joint two-layer (IP layer and optical layer) token based FDL scheme was proposed in a bottom-up fashion [34]. Such a FDL scheme starts from optical layer, which is closer to the network fault, and is taken over by IP layer if optical layer cannot handle the fault. The fault detection and localization at the optical layer relies on monitoring devices residing in the network nodes, while the similar functionality is carried by routing protocols or MPLS in the IP layer. The interaction and influences between the FDL in two layers were studied: the impacts of holdoff timer before the FDL function is passed from optical layer to IP layer, and alternatively, the optical layer explicitly sends recovery token to trigger IP layer FDL [34]. 


\subsection{Optical layer fault detection and localization}

FDL approaches at the physical layer include network-model based FDL, black-box learning-based FDL, and hybrid methods.

\subsubsection{Network-model based FDL}

Network-model based FDL approaches firstly build a network model according to the functional and physical characteristics of network components, transmission properties, as well as network topologies. Such network models are usually established off-line before the network operation, or at least before the running of the FDL procedure. Such off-line modeling work could significantly reduce the FDL time and lead to complex and accurate models. On the basis of the pre-established network model, the FDL system could forecast the behaviour of the given network and compare with the actual observations in the real network operation. Any discrepancy between the prediction and the observation implies the occurrence of one or more network faults. Figure 2.5 shows the working theory of an abstract network-model based FDL system [16].

The most important advantage of network-model based FDL is that they can cope with unforeseen faults. Furthermore, the speed of network-model based FDL is the fastest among all kinds of FDL approaches. Additionally, they can even work with incomplete fault alarm information. The main drawback of network-model based FDL is the difficulty of developing good models for complicated networks.

The network-model based FDL approaches include the following reported work: 


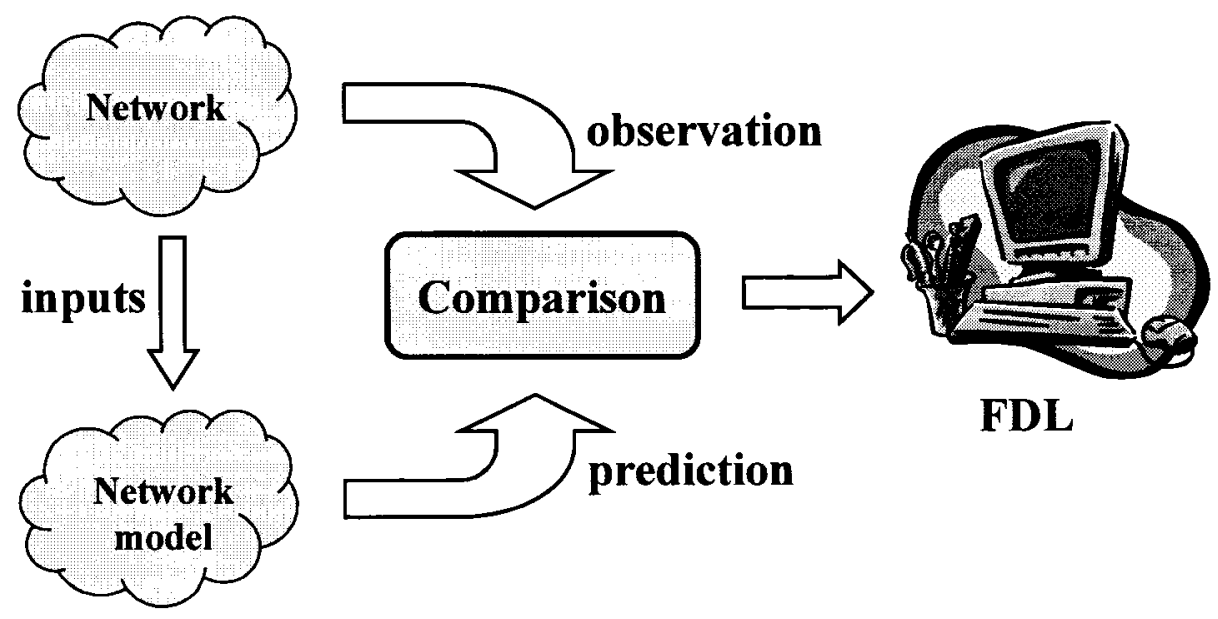

Figure 2.5 Network-model based fault localization

\section{1) Finite state machine (FSM) model}

The finite state machine (FSM) model consists of a set of states, together with the transition rates between states. Each state represents either a unique working status of the network [22], or a status of a link [23]. Network events such as alarms make the network or a link transits from one state to another one. Thus each network fault scenario is represented by a unique state and such FDL approaches can only identify the fault scenarios considered in the FSM model. The FSM-model can achieve accurate and highspeed FDL. However, it is difficult to enumerate all possible failure scenarios for a large or even moderate-size network, especially for AONs providing dynamical lightpath provisioning.

\section{2) Probabilistic reasoning systems}

In probabilistic FDL systems, a failure probability is assigned to each network component (e.g., nodes and links). The conditional failure probabilities of an element 
given the failure of another one are also set as the model parameters. The failure propagation probabilities based on the network topology and transmission properties are also included in the model $[17,80-81,103]$. As a kind of network-model based FDL approaches, probabilistic reasoning systems can cope with unforeseen fault scenarios. However, failure probabilities of network components are required for building the model, which is not always available. Especially, the conditional failure probabilities may be extremely difficult to determine because of the time dependency in optical channels (lightpaths). Furthermore, such probabilities might change all the time due to the dynamically lightpath provisioning, which would put huge expense in computing and refreshing the failure probabilities in the model, if such dynamic refreshment is not impossible.

\section{3) Deterministic Modeling Algorithms}

Deterministic network models are established on the basis of properties of network components. One proposal of the early work in this area firstly assigns a network monitor (e.g., OPM) to each established lightpath in an optical network [37]. It then builds a faultreporting alarm matrix that correlates every potential faulty node (row) with every monitor (column). By removing the zero-row and repeated (identical) columns in the alarm matrix, the number of monitors could be minimized. The method is practical but the performance highly depends on the location of assigned monitors. Furthermore, assigned monitors can hardly be re-located and consequently hard to support dynamic lightpath provisioning. Even if monitor-relocation is possible, dynamic lightpaths will invoke huge re-calculation of alarm matrix. 
Another network model reported in $[21,36]$ classifies optical components in an AON into the following categories according to the capability of masking network failures, while masking a network fault means blocking its propagation in the network:

$O_{0}$ : Optical components that cannot mask any failure, e.g., optical fiber;

$O_{1}$ : Optical components that can mask all kinds of failures, e.g., optical regenerator;

$\mathrm{O}_{2}$ : Optical components that can mask out-of-band jamming (intrachannel or interchannel crosstalk), e.g., optical filter;

$\mathrm{O}_{3}$ : Optical components that can mask power drops, e.g., optical amplifier.

The monitoring components are also classified based on the failures they can detect:

$V_{1}$ : Monitoring components can only detect the power variations, e.g., OPM;

$V_{2}$ : Monitoring components can detect the power variations and out-of-band jamming, e.g., OSNR;

$V_{3}$ : Monitoring components can detect the power variations, out-of-band jamming, and in-band jamming, e.g., BER;

$V_{4}$ : Monitoring components can detect the power variations and wavelength misalignment, e.g., OSA.

Wavelength channels (lightpaths) are then modeled as an ordered set of optical elements and monitoring components. A binary tree is built with a depth equals to the number of monitoring components, and each level of the tree corresponds to a monitoring component in the network. Whenever a failure occurs, it triggers alarms in some monitoring components but not in others. Each network failure then generates a binary alarm vector, in which every component is the indicator of alarm for a monitoring 
component. The faulty source can be located via traversing the binary tree and finding the matched route from the root to a leaf.

One more proposed model is built according to the propagation characteristics of crosstalk attacks, which is considered as a kind of network fault in wide sense[38-39, 82]. The reported scheme employs status of connections as diagnostic data, and thus places a relatively small number of monitors on a selected set of nodes in a network to achieve the required level of performance. The authors proved that it is not necessary to assign monitors to each node for detecting crosstalk attacks. Authors assessed a simple monitor placement policy: a neighbor of a degree one node and all neighbors of a non-monitor node must have at least one monitor. Such a monitor placement policy is proven to be effective. However, since the monitor is connection based, multiple monitors may be required within one node (according to the author). This drawback impedes the further reduction of monitor costage. More seriously, for dynamic lighgtpath provisioning (equivalent to connection setup), the assigned monitors can hardly be relocated to new connections. Therefore it makes such a scheme extremely difficult, if not impossible.

The deterministic models based FDL need small amount of information e.g., no failure probabilities are needed, and no prior knowledge needed except for optical channels. They are also tolerant to false and missing alarms in the network. However, each failure scenarios must be known and pre-defined in such FDL approaches thus large amount of pre-computation and storage is required. In some cases, the model would be too complex to be implemented, esp. for large-size AONs providing dynamical lightpath provisioning. 


\subsubsection{Black-box learning-based FDL}

Other than the network-model, the black-box learning-based FDL approaches consider the network as a black box that delivers some certain output data when a particular network fault occurs. Black-box based FDL approaches usually include the learning phase and operational phase. In the learning phase, the system explores and records the relationship between input network events and the output fault diagnosis. In the operational phase, the system outputs the fault diagnosis based on the knowledge obtained in the previous learning phase. Due to the existence of the learning phase, the FDL time is usually larger than the network-model based approaches. Moreover, a network fault that does not occur in the initial learning phase will invoke a new learning action and thus further increase the FDL time. The advantage of the black-box approaches is that they do not need to know the detail of the network, e.g., network topology and components. They are very appropriate for the case that network details are not available.

Black-box learning-based FDL can be done in different methods: human expert systems, case-based systems, artificial neural networks (ANN), and any other statistical learning algorithms such as proactive learning systems.

\section{1) Expert Systems}

Expert systems are established based on the human experts' knowledge of the network. The system firstly generates a set of rules representing the relationship between a network event (alarm) and a fault hypothesis. Then the expert system extracts a fault hypothesis from the rule database once a network event occurs. Expert systems can 
effectively integrate the human expertise into the FDL system and are easy to cope with large-scale networks. However, the relationship between fault symptoms and fault hypothesis (rules) need to be exhaustively pre-defined, which may lead to a large number of rules and consequently, huge database. The FDL time would also be large due to the exhaustive search for all possible fault hypotheses. Furthermore, any change of network components and connections will make the expert system change dramatically, especially in AONs supporting dynamical lightpath routing and switching.

\section{2) Case-Based Systems}

Case-based systems remember the fault scenarios solved in the past, and make the FDL decision in the future by retrieving the previous cases. Case-based systems show the high efficiency and speed when the fault scenario is a previously solved case. New fault scenarios would also be saved as a case in the database in the online learning system. The implementation of case-based system is relative simple comparing with other FDL systems. However, the case-based systems need huge storage for past cases and the FDL time will dramatically increase for new fault scenarios that were not previously solved. It would take many years to build up enough cases by experience when it comes to physical layer failures. In absolute terms fibre cuts are quite rare event in the real world, so building up cases by experience is too time-consuming. Also, the service provider cannot afford to let failures happen and not be pre-prepared to restore services instantly. Additionally, in dynamical routing and switching AONs, due to the change of network connections, the previous cases might not be suitable for the same case met in the future. Such cases would lead to inaccuracy in FDL. Therefore, case learning is not an option. 


\section{3) Artificial Neural Networks (ANN)}

An artificial neural network consists of a set of neurons (nodes) and weights (links). The neurons are connected by weights, whose values are initially assigned randomly or by some heuristics. In the statistical learning phase, the ANN updates the values of weights by learning sample pairs between network events and desired outputs. In the operational phase, the ANN provides correct FDL results whenever a fault scenario occurs. There is no network modeling is needed for ANN-based FDL. If the training is good enough, i.e., the ANN generalized good enough weight values, it can cope with new network fault cases, even with false and missing alarms. However, the training phase is slow and the generalization to new cases could be poor. Moreover, for dynamical AONs (lightpath routing and switching), the changed logical topology of the network may needs a new learning phase, which may dramatically increase the FDL time.

\section{4) Proactive Learning Systems}

The proactive FDL systems continuously monitor and analyze the performance of the network, in order to detect any performance degradation or anomalies, and consequently forecast potential network faults before they really occur. This is the derivation of the term "proactive". The proactive learning systems set dynamic threshold of performance indices as described in Section 2.1 by studying the variation of performance measurement under normal network operation. Such methods are sensitive to most network anomalies and can cope with unforeseen network faults. However, proactive learning systems need to continuously study the normal behaviour of the network, especially for dynamical 
switching and routing AONs. Additionally, the output of such FDL systems is only warning information, rather than accurate located faulty source, for any network anomalies.

\subsubsection{Hybrid methods}

It is possible to combine two or more above methods into one FDL system. For example, reference [83] proposed a probabilistic method based on a Bayesian network model, together with a proactive learning system for setting parameters. Hybrid FDL approaches can take the advantages of each single method integrated within it. However, they could not avoid all disadvantages of all methods included in the model.

\subsection{Comparison of FDL mechanisms}

FDL approaches in the IP layer and at the physical layer have their respective advantages and disadvantages. FDL approaches for AON in the IP layer are usually practical and cost-effective, due to the fact that the function of FDL has already been integrated within most existing IP-layer protocols. Even most newly proposed dedicated FDL protocols in IP layer can also be implemented via customizing the existing IP-layer protocols. Not as in network-model FDL approaches, the knowledge of the network is not necessary for IP-layer FDL protocols. Such simplicities of implementation greatly reduce the cost for FDLs. The IP-layer approaches detect and locate network faults at the fine granularity of a single lightpath or even a part of a lightpath (e.g., the label switching path 
- LSP). Furthermore, IP-layer FDLs support dynamic lightpath provisioning and are scalable respect to the network size. However, the long fault detection and localization time in IP layer (typical at seconds-level or sub-second level) makes it difficult to achieve time-critical recovery. Additionally, while the small FDL granularity provides flexibility of FDL, it puts huge overhead upon the network because of a large amount of alarms and notifications. Therefore, some effective and efficient FDL mechanisms at physical layer are expected.

At the physical layer, the black-box based approaches do not need to build network models and thus simplified the FDL procedure. They are efficient, scalable in terms of network size, and can usually cope with previously not-experienced network faults by adding the new fault to the fault correlation database through learning or training processes. But the slow learning/training process makes the FDL slow, especially at the beginning of the network operation while most faults are previously not-experienced. Moreover, whenever the lightpaths change in an $\mathrm{AON}$, they have to invoke the learning/training process to update the previously established faults database. In an AON providing dynamic lightpath provisioning, such updates bring a large amount of computing load and significantly slow down the FDL speed.

Network-model based approaches can achieve accurate FDL results and can cope with unforeseen faults without any learning or training process, thus greatly accelerating the FDL time. FSM models enumerate all fault scenarios via defining one in each state in the state machine. They obtained the fastest and most accurate FDL among all FDL approaches. But it is difficult, if not impossible, to enumerate all possible failure scenarios, especially for large-scale, complicated AONs. They bring a growing amount of 
states and (real time) computation load along with the increase of network scale, thus slow down the FDL time to an un-tolerable level. Probabilistic reasoning systems are relatively easier to be built than FSM models. But it is difficult to determine failure propagation probabilities and failure probabilities, especially the dependent failure probabilities for network components. Deterministic models can achieve fast and accurate FDL results and do not need to know any prior failure probabilities. The main drawback of deterministic models is the complexity of developing good models for complicated networks. But the construction of such models can be carried in an off-line procedure to avoid the time-consuming model computing in the real time FDL process. Since the time is not critical for building network models, the network model can be greatly improved to achieve more accurate FDL results. Therefore, the deterministic network based FDL approaches are the most premising. The work of this thesis will focus on the physical layer FDL approaches based on deterministic network-models to achieve fast and accurate results. 


\section{Chapter 3}

\section{Monitoring-Cycle}

\subsection{Network Fault Classification}

$\mathrm{I}$

$\mathrm{N}$ our AON model a link is actually a single fibre, in which multiple optical channels are multiplexed. Note that the impact scope of a network fault is various in term of optical channels (equivalently, lightpaths or wavelengths). Some network faults only affect a single or some specific channels going through the faulty network element, e.g., the transmitter laser failure and $\mathrm{OXC}$ port blocking. On the other hand, some others may affect all channels that pass through the faulty module, e.g., fibre cuts and the optical amplifier saturation. The former is defined as type I network faults and the latter type II in this thesis. The characteristics of AON faults are strongly related to the specific network components [57]. For AONs, type I faults usually can be handled by in-place fault control and management entities, or some channel-oriented monitoring techniques such as the pilot-tone based methods reported in $[52,58]$. On the other hand, type II network faults put an impact on more user traffic and generate much more alarms in the network, thus they degrade the network performance more severely. Furthermore, type II faults occur more frequently in the real world, e.g. about one-third of the total network failures are fibre cut, as reported in [48, 59], which affect all related channels and users. 
Therefore it is critical to develop some mechanisms to address type II network faults.

Table 3.1 lists a collection of faults for key AON components, as well as their impact scopes and existing fault detection methods. The work in this thesis will focuses on the type II faults.

Table 3.1 Network fault classification and detection in AONs

\begin{tabular}{|c|c|c|c|}
\hline Component & Detectable fault & Detection methods & Impact scope \\
\hline \multirow{6}{*}{$\begin{array}{l}\text { Optical } \\
\text { Amplifier }\end{array}$} & Temperature out of range & Temperature sensor & Type II, all wavelengths \\
\hline & Loss of power/signal & $\begin{array}{l}\text { OPM, signal } \\
\text { supervisory }\end{array}$ & Type II, all wavelengths \\
\hline & $\mathrm{I} / \mathrm{O}$ power out of range & $\begin{array}{l}\text { OPM } \\
\text { (aggregate/channel) }\end{array}$ & $\begin{array}{l}\text { Type II, specific/all } \\
\text { wavelength }\end{array}$ \\
\hline & Pump power loss & \multirow{2}{*}{$\begin{array}{l}\text { Pump monitoring } \\
\text { device, OPM } \\
\text { (aggregate/channel) }\end{array}$} & Type II, all wavelength \\
\hline & Pump saturates & & Type II, all wavelength \\
\hline & Noise, dynamic gain & EDC, BER monitoring & Type II, all wavelength \\
\hline \multirow{6}{*}{$\begin{array}{l}\text { MUX/ } \\
\text { DEMUX }\end{array}$} & Temperature out of range & Temperature sensor & Type II, all wavelengths \\
\hline & Loss of power/signal & $\begin{array}{l}\text { OPM, signal } \\
\text { supervisory }\end{array}$ & Type II, all wavelengths \\
\hline & $\mathrm{I} / \mathrm{O}$ power out of range & $\begin{array}{l}\text { OPM } \\
\text { (aggregate/channel) }\end{array}$ & $\begin{array}{l}\text { Type II, specific/all } \\
\text { wavelength }\end{array}$ \\
\hline & Increased insertion loss & $\begin{array}{l}\text { OPM } \\
\text { (aggregate/channel), } \\
\text { OSNR }\end{array}$ & Type II, all wavelength \\
\hline & Increased crosstalk & Via BER & Type II, all wavelength \\
\hline & Increased noise & EDC, BER monitoring & Type II, all wavelength \\
\hline \multirow{3}{*}{$\begin{array}{l}\text { OXC } \\
\text { (MEMS) }\end{array}$} & Temperature out of range & Temperature sensor & Type II, all wavelengths \\
\hline & $\begin{array}{l}\text { OCh loss (one/few/all } \\
\text { OChs) }\end{array}$ & $\begin{array}{l}\text { OPM, signal } \\
\text { supervisory }\end{array}$ & $\begin{array}{l}\text { Type I, specific/all } \\
\text { wavelengths }\end{array}$ \\
\hline & I/O power out of range & $\begin{array}{l}\text { OPM } \\
\text { (aggregate/channel) }\end{array}$ & $\begin{array}{l}\text { Type II, specific/all } \\
\text { wavelength }\end{array}$ \\
\hline
\end{tabular}




\begin{tabular}{|c|c|c|c|}
\hline Component & Detectable fault & Detection methods & Impact scope \\
\hline & Increased insertion loss & $\begin{array}{l}\text { OPM } \\
\text { (aggregate/channel), } \\
\text { OSNR }\end{array}$ & Type II, all wavelength \\
\hline & $\begin{array}{l}\text { Port blocking: Connection } \\
\text { established }\end{array}$ & Upper layer & Type I, specific wavelengths \\
\hline & Unavailable resources & Upper layer & Type I, specific wavelengths \\
\hline & Unauthorized request & Upper layer & Type I, specific wavelengths \\
\hline & Misrouting & Upper layer & Type I, specific wavelengths \\
\hline \multirow{6}{*}{ Transmitter } & Temperature out of range & Temperature sensor & Type I, one wavelength \\
\hline & Output power degradation & OPM & Type I, one wavelength \\
\hline & Output power loss & OPM & Type I, one wavelength \\
\hline & Wavelength drift & $\begin{array}{l}\text { OSA, OSNR monitor, } \\
\text { EDC }\end{array}$ & Type I, one wavelength \\
\hline & Line-width broadening & EDC, OSA & Type I, one wavelength \\
\hline & Modulation depth change & EDC, BERT & Type I, one wavelength \\
\hline \multirow{5}{*}{ Receiver } & Input power out of range & OPM & Type I, one wavelength \\
\hline & LOP/LOS & $\begin{array}{l}\text { OPM, signal } \\
\text { supervisory }\end{array}$ & Type I, one wavelength \\
\hline & Temperature out of range & Temperature sensor & Type I, one wavelength \\
\hline & $\begin{array}{l}\text { Gain/speed degradation } \\
\text { (aging) }\end{array}$ & BERT & Type I, one wavelength \\
\hline & Ceases to detect photons & $\begin{array}{l}\text { Electrical signal } \\
\text { supervisory }\end{array}$ & Type I, one wavelength \\
\hline \multirow[t]{2}{*}{ Fibre } & Fibre cut & $\begin{array}{l}\text { OPM, signal } \\
\text { supervisory }\end{array}$ & Type II, all wavelengths \\
\hline & Fibre stress/bend & OSNR, BERT & Type II, all wavelengths \\
\hline
\end{tabular}




\subsection{Monitoring-Cycle}

In this thesis a novel approach is proposed for detecting and locating type II network faults in mesh AONs. The basic idea is to decompose an AON into a set of cycles, in which each node and link appears at least once, so that all these cycles form a cycle cover for the given AON. The cycle cover is defined as a set of cycles in a given graph such that all links and nodes in the graph appear in atleast one cycle in the set. A network monitor is assigned to one node in each cycle and a loopback supervisory channel is set up in this cycle. A cycle with the monitor and the supervisory channel is defined as the "monitoring cycle (m-cycle)". Depending on the type of monitors in m-cycles (e.g. optical power meters, optical spectrum analyzers, optical transceivers, etc.), various performance indices of AONs described in Chapter 2.1 can be measured. Flexible index thresholds can be set to determine whether a network fault occurs. A network fault can be a level of degradation in any of the performance indices such as optical power, optical spectrum, OSNR, or more fundamentally, bit error ratio (BER). The assigned network monitors in $m$-cycles can measure all above performance indices, not only some specific signaling such as the loss of light (LOL) or loss of signal (LOS) as in $[35,37,60]$. Additionally, the loopback supervisory scheme puts the transmitter and receiver together in a single node in an $m$-cycle and brings potential benefits, for example, the optical source signal may work as the reference for the received signal for the purpose of comparison. The $m$-cycle based fault detection and localization method is referred as MFDL in this thesis.

In the MFDL design, a network fault triggers alarms in the $m$-cycles in which it 
appears, but not in others. All alarms are collected in real time by either centralized or distributed network management unit (NMU). Thus any network fault is detectable and the potential faulty source is located to the common part of those $m$-cycles with alarms. The centralized fault localization methods are more efficient than their distributed counterparts and are compatible to the current OIF SDH/SONET architectures. Additionally, NMU failure is rare under the current technologies and environments so that the single-point failure of the central server is not a problem. Microsoft $\otimes$ reported that the centralized management architecture is much more acceptable for large enterprises, e.g., $60 \%$ of the 500 largest enterprises will attain the centralized management architecture by 2006 [120]. Therefore, in this thesis, the discussion is mainly focused on the centralized NMU, although the distributed NMU for MFDL is also possible.

A mesh AON can be modeled as a finite undirected graph $G(V, E)$, where $V$ is the set of vertices (network nodes) and $E$ is the set of edges (network links). The use of vertex (edge) and node (link) is exchangeable in the context of this thesis. As defined at the beginning of Chapter 3, a link in this thesis represents a single fibre that contains multiple optical channels with DWDM technology. It is also assumed in this thesis that such a graph is 2 -connected and it contains neither loops ${ }^{*}$ nor multiple edges ${ }^{\dagger}$. A cycle (denoted as $c$ ) of graph $G$ is a sub-graph of $G$ that is connected and regular of degree two [61]. To avoid allowing $K_{2}$ (the complete graph of a single edge and its two endpoints) being a cycle, the number of vertices in a cycle must be at least three. If graph $G$ becomes disconnected from connected after deleting a single edge $e$, then edge $e$ is defined as a

\footnotetext{
* A loop is an edge that starts and ends at the same vertex in a graph.

${ }^{\dagger}$ The multiple edges refer to parallel edges that have the same start-point and end-point.
} 
bridge of $G$ (or cut-edge). More general, a bridge is an edge whose deletion increases the number of components of graph $G$ [61]. A cycle is often identified either with its edge-set or with its vertex set. A cycle cover (denoted as $C$ ) of a graph is a set of cycles such that each vertex and edge of the graph appears in at least one of these cycles. If an edge $e$ or vertex $n$ appears once in a cycle $c, c \in C$, It is said that edge $e$ or vertex $n$ is covered by cycle $c$ and their cover depths in $C$ increases one respectively. Figure 3.1 gives such a graph example with 10 nodes and an instance of its cycle covers, $C=\left\{c_{1}, c_{2}, c_{3}, c_{4}\right\}$. Some nodes and links appear only in one cycle of $C$. For example node 3 , links $2-3$ and 3-7, are covered by cycle $c_{4}$ only. But some other nodes and links appear in multiple cycles, e.g. edge $2-4$ is covered by cycles $c_{1}$ and $c_{4}$, node 6 by cycles $c_{2}, c_{3}$ and $c_{4}$.

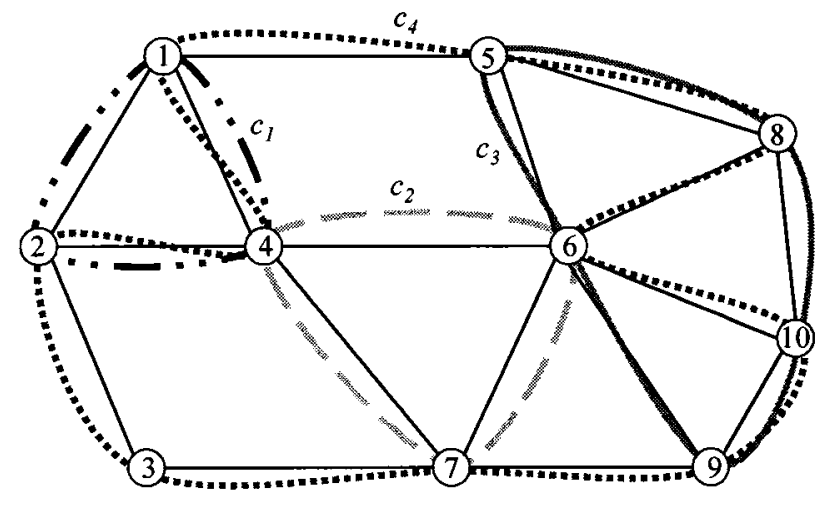

Figure 3.1 A graph example and its cycle cover instance

A connected graph without bridge edges (called bridgeless) can be decomposed into a set of cycles. The feasibility of such decomposition is based on the cycle double cover conjecture [62]:

Cycle Double Cover Conjecture (CDCC): Every bridgeless graph has a cycle $-45-$ 
double cover, which is a cycle cover such that every edge of the graph appears in exactly two of those cycles in the cover.

Although the conjecture has not been completely proven, it was shown in [62-63] that a minimum counterexample to the cycle double cover conjecture must be a snark that has girth at least seven or more. A snark is a cyclically 4-edge-connected cubic graph with girth $\geq 5$, where the girth of a graph is the length of the smallest cycle contained in the graph [64]. Please note that no snark with girth $\geq 7$ is known. In fact, some literatures such as [65] had conjectured that such snarks do not exist. That is to say, there exist only snarks with girth of 5,6 , or 7 . Thus, it is safe to say, even if the counterexamples to the conjecture do exist, it is not expected that communication networks with such topologies will be encountered in the real world.

The cycle double cover conjecture indicates that it is possible to fully detect all faults by using at most two wavelengths within each link. It therefore not only shows the feasibility of using $m$-cycles for fault detection but also gives a reference for evaluating the performance of $m$-cycle construction algorithms regarding the network resource overhead. A good $m$-cycle constructing algorithm should have an overhead close to two wavelengths per link.

Note the condition of the cycle double cover conjecture: there is no bridge in the given graph. Depending on whether a network is bridgeless or not, there exist the following different strategies to apply the MFDL approach:

Strategy 1: For a bridgeless AON, assign a network monitor to one of the nodes in each $m$-cycle and a loopback dedicated supervisory channel (using a single wavelength) for each $m$-cycle. Once a type II fault occurs on the paths covered by some $m$-cycles, it 
triggers an alarm in each of these cycles and thus it is detectable. To further localize the fault, a fault localization algorithm is required.

For a graph containing some bridge edges, one can remove all the bridge edges and thus divide the graph into some bridgeless sub-graphs.

Strategy 2: If the topology of an AON contains bridge links, assign a network monitor to each bridge link and remove this link, the remainder of the topology will be a family of some bridgeless sub-graphs. Apply strategy 1 to each bridgeless sub-graph. Then all type II network faults are detectable.

In reality, a bridge link is a single-failure point for the whole network, thus such links are usually avoided during the network topology design stage. Theoretically, one cannot find any cycle in a tree or forest, which is considered as an acyclic graph. Each link in an acyclic graph can be considered as a bridge. Therefore, if strategy 2 is applied to an acyclic graph, each link of it will be assigned a network monitor and consequently it shrinks to the monitor-per-link case.

If a "mesh AON" is defined as a finite connected graph (either undirected or directed, although only undirected graphs are considered in this thesis), which contains no loops, no multiple edges, the following theorem is claimed,

Theorem 3.1: In a mesh AON, all type II network faults can be detected by applying either strategy 1 or strategy 2. 


\subsection{M-Cycle Based Fault Localization Algorithm} (MFLA)

The target of fault localization is to determine the faulty elements once a network fault occurs. The faulty elements are expected to be clearly identified before any actions for recovery. With the $m$-cycle based FDL approach, once any fault occurs along a network link, it will trigger alarms in $m$-cycles that cover the link, but not in others. For example, Figure 3.1 gives a graph example with the $m$-cycle set $C=\left\{c_{1}, c_{2}, c_{3}, c_{4}\right\}$. If a fault occurs along link $(1,4)$, it will trigger alarms in both $m$-cycles $c_{1}$ and $c_{4}$, but there is no alarm in other $m$-cycles. Conversely, if alarms are received in $m$-cycles $c_{1}$ and $c_{4}$ but no other $m$-cycles, it implies that the potential faulty links could be the common part of $m$ cycles $c_{1}$ and $c_{4}$, i.e., either $(1,4)$ or $(2,4)$.

Given a graph $G(V, E)$ with $L$ links, $N$ nodes and $M m$-cycles, for any link $e_{i} \in E(i=1,2, \cdots, L)$ and any $m$-cycle $c_{j}$, a binary associative bit, $a_{i j}$ is defined as,

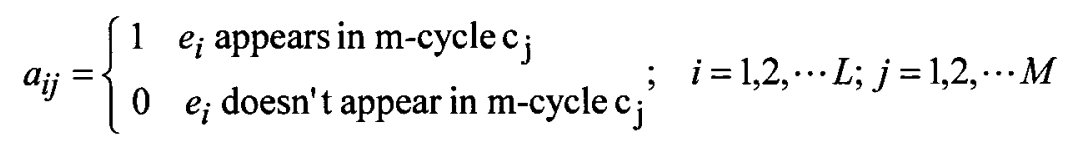

For any link $e_{i}$, the sequence of its associative bits corresponding to all the $m$-cycles is defined as the associative code $\mathbf{a}_{i}=\left(a_{i 1}, a_{i 2}, \cdots, a_{i M}\right)$ ( $M$ bits in total). Each link has its own associative code. Furthermore, a binary status indication bit $m_{j}$ is defined for each $m$-cycle $c_{j}$, to indicate the status of $c_{j}$, i.e. whether or not a fault occurs and thus an alarm is triggered in $c_{j}$, 


$$
m_{j}=\left\{\begin{array}{l}
1 \quad \text { an alarm is generated in } \mathrm{m}-\operatorname{cycle~}_{\mathrm{j}} \\
0 \quad \text { no alarm is generated in } \mathrm{m}-\operatorname{cycle~c}_{\mathrm{j}}
\end{array} ; \mathrm{j}=1,2, \cdots M\right.
$$

The sequence of alarm indication bits for all $m$-cycles is defined as an alarm code $\mathbf{m}=\left(m_{1}, m_{2}, \cdots, m_{M}\right)(M$ bits in total $)$. A NMS collects alarms in $m$-cycles and generates alarm-codes in real time.

Based on the above definitions, the MFLS can be described as the following,

Once alarms in $m$-cycles are collected and an alarm code is generated, the alarm code is compared bit by bit with the associative code for each link. If a link's associative code exactly matches the alarm code, then this link is a faulty candidate for the received alarm code. Such a comparison can be implemented by the per-bit "exclusive-OR $(\oplus)$ " operation between the alarm and the associative code. A logic variable, $F_{i}$ is calculated for link $e_{i}$ as the faulty candidate flag while receiving a new alarm code $\mathbf{m}$,

$$
F_{i}=<\mathbf{a}_{i}, \mathbf{m}>=\sum_{j=1}^{j=M} a_{i j} \oplus m_{j} \quad i=1,2, \cdots L
$$

Where $\langle\bullet, \bullet\rangle$ is the inner product of two vectors [66]. If $F_{i}=0$, link $e_{i}$ is a faulty candidate for the received alarm code $\mathbf{m}=\left(m_{1}, m_{2}, \cdots, m_{M}\right)$. After checking associative codes for all links, a faulty candidate set can be obtained for any real-time alarm code. For instance, for the network example shown in Figure 3.1 with the $m$-cycle set $C=\left\{c_{1}, c_{2}, c_{3}, c_{4}\right\}$, the associative bits and corresponding associative codes are listed in Table 3.2. By applying the MFLA and calculating formulae 3.3.3 for all possible combinations of alarm bits for $m$-cycles $c_{1}, c_{2}, c_{3}$, and $c_{4}$, the faulty candidate set for each alarm code is established in Table 3.3, e.g. link $(1,4)$ appears in both $m$-cycles $c_{1}$ 
and $c_{4}$ thus its associative code is 1001 . As discussed in the beginning of this subsection, for example, for the alarm code 1001, i.e. alarms are generated in $m$-cycles $c_{1}$ and $c_{4}$ but not in any other $m$-cycles, the faulty candidate would be the common part of these two $m$ cycles: links $(1,4)$ and $(2,4)$. Please note that there exists only a single faulty candidate for some alarm codes, while multiple candidates for others.

Table 3.2 Associative bits and codes for the network example in Figure 3.1

\begin{tabular}{c|cccc}
\hline \multirow{2}{*}{ Link } & \multicolumn{4}{|c}{ Associative Code } \\
\cline { 2 - 5 } & $c 1$ & $c 2$ & $c 3$ & $c 4$ \\
\hline$(1,2)$ & 1 & 0 & 0 & 0 \\
\hline$(1,4)$ & 1 & 0 & 0 & 1 \\
\hline$(1,5)$ & 0 & 0 & 0 & 1 \\
\hline$(2,3)$ & 0 & 0 & 0 & 1 \\
\hline$(2,4)$ & 1 & 0 & 0 & 1 \\
\hline$(3,7)$ & 0 & 0 & 0 & 1 \\
\hline$(4,6)$ & 0 & 1 & 0 & 0 \\
\hline$(4,7)$ & 0 & 1 & 0 & 0 \\
\hline$(5,6)$ & 0 & 0 & 1 & 0 \\
\hline$(5,8)$ & 0 & 0 & 1 & 1 \\
\hline$(6,7)$ & 0 & 1 & 0 & 0 \\
\hline$(6,8)$ & 0 & 0 & 0 & 1 \\
\hline$(6,9)$ & 0 & 0 & 1 & 0 \\
\hline$(6,10)$ & 0 & 0 & 0 & 1 \\
\hline$(7,9)$ & 0 & 0 & 0 & 1 \\
\hline$(8,10)$ & 0 & 0 & 1 & 0 \\
\hline$(9,10)$ & 0 & 0 & 1 & 1 \\
\hline & & & & \\
\hline
\end{tabular}


The $m$-cycle based fault localization algorithm (MFLA) enumerates all possible alarm codes and pre-establishes a table of corresponding faulty candidate sets offline. Then in real time the NMU only looks up the table and finds the corresponding faulty candidate set when a new alarm code is generated. Such a localization algorithm is fast and has logarithmic complexity in real time running. Furthermore, although the above MFLA is focused on the single-fault scenarios, it could be extended to multi-fault scenarios. For example, if faults occur on links $(1,5)$ and $(4,6)$ in Fig. 3.1 at the same time, alarms will be observed in both $m$-cycles $c_{2}$ and $c_{4}$. A new alarm code not in Table 3.3 is then generated: 0101. The MFLA results for it should be the link combination $(1,5)$ and $(4,6)$. In this way, the sources of double-fault scenarios could be localized through including all corresponding alarm codes and their faulty candidates in the pre-established fault localization result table. Similarly, a network node can also generate an alarm in the $m$ cycle that covers the node. An associative bit can also be assigned to each network node,

Table 3.3 MFLA results for the network example in Figure 3.1

\begin{tabular}{cccc|c}
\hline \multicolumn{3}{c|}{ Alarm Code } & Faulty Candidates \\
\hline$c 1$ & $c 2$ & $c 3$ & $c 4$ & Null \\
\hline 0 & 0 & 0 & 0 & $(1,5),(2,3),(3,7),(6,10),(7,9),(6,8)$ \\
\hline 0 & 0 & 0 & 1 & $(5,6),(6,9),(8,10)$ \\
\hline 0 & 0 & 1 & 0 & $(5,8),(9,10)$ \\
\hline 0 & 0 & 1 & 1 & $(4,6),(4,7),(6,7)$ \\
\hline 0 & 1 & 0 & 0 & $(1,2)$ \\
\hline 1 & 0 & 0 & 0 & $(1,4),(2,4)$ \\
\hline 1 & 0 & 0 & 1 & N/A \\
\hline
\end{tabular}




$$
b_{i j}=\left\{\begin{array}{ll}
1 & \text { node } i \text { appears in } \mathrm{m} \text {-cycle } \mathrm{c}_{\mathrm{j}} \\
0 & \text { node } i \text { doesn't appear in } \mathrm{m} \text {-cycle } \mathrm{c}_{\mathrm{j}}
\end{array} ; i=1,2, \cdots L ; j=1,2, \cdots M\right.
$$

The node-fault scenario could then be incorporated in MFLA by matching alarm codes with node associative codes and including the MFLA results for network nodes in the pre-established fault localization table.

\subsection{A Lower Bound of $M$-Cycle Number for Complete}

\section{Localization}

For MFLA, each faulty candidate set contains only a single network element in ideal case. However, typically multiple candidates exist in a single set due to the fact that $m$ cycles are not constructed to minimize such candidate set, e.g., candidate sets for some alarm codes in Table 3.3. In the survivable AON operation, the number of candidates in each set must be kept as small as possible for fault recovery. If the number of faulty candidates is small, we can either further localize the faulty source by in-place alarms while the minimum candidate set reduces the costs of in-place monitoring, or shift the traffic on all the paths that contain one or more faulty candidates to backup paths that bypass all the faulty candidates. If the faulty candidate set contains no more than a single element for every alarm code, it is said that the complete fault localization is achieved.

Based on the MFLA described in Section 3.3 (see equation 3.3.3), a necessary condition for achieving the complete fault localization is that each link must have a 
unique associative code. Let $L$ be the number of links in a graph, and $M$ be the number of $m$-cycles in a cover set of the graph, then each associative code includes $M$ bits and the number of the total possible associative codes must be larger than $L$ to meet the necessary condition. Since the all-zero code is not usable (a link is covered by at least one cycle), so $2^{M}-1 \geq L$, i.e.,

$$
M \geq \log _{2}(L+1)
$$

Inequality (3.4.1) gives a lower bound on the number of $m$-cycles for achieving complete fault localization. It can be used as a lower bound on the cost of network monitors and wavelengths required for monitoring to completely localize all network faults based on $m$-cycles in AONs. However, since the associative code cannot be randomly selected in real network topologies, usually this bound is quite loose.

For the ideal case, each possible associative code is used exactly once for a unique link ( $L=2^{M}-1$, i.e. $\left.M=\log _{2}(L+1)\right)$. Then each associative code is unique and a complete localization will be achieved for any alarm code. Note the fact that at each bit position, the values are " 0 " in the half of all the associative codes and " 1 " in the other half. The average link cover depth (equivalent to the average reserved wavelengths for monitoring in a link) can be estimated as,

$$
\frac{M \times(L+1) / 2}{L} \approx \frac{1}{2}\left\lceil\log _{2}(L+1)\right\rceil
$$

The link cover depth equals to the number of $m$-cycles in which the link appears. This is the minimum average cover depth for a link to achieve complete localization. Formula 3.4.2 also gives the lower bound of wavelength cost for achieving the complete fault localization in MFDL approaches. 


\subsection{Performance Evaluation Metrics}

To evaluate the performance of $m$-cycle based FDL approaches, the following group of metrics is proposed in this section: the localization degree, maximum and average wavelength overhead, as well as cost reduction. The localization degree is the measurement of the quality of the MFLA results. As described in the definition of $m$ cycle, some wavelengths may be reserved for monitoring in network links and thus cannot be used for user data. The wavelength overhead measures the network resources utilization with regard to wavelengths. The wavelength overhead is important because it might be high in some reported FDL schemes. The cost of MFDL includes not only the expense of monitoring devices, but also reserved wavelengths.

\subsubsection{Localization degree}

As shown in Table 3.3, the faulty candidate set for an alarm code could contain either single or multiple elements. The number of elements could be reduced for the latter by adding extra cycles to the $m$-cycle set. Furthermore, the average number of elements for candidate sets could be various by applying different $m$-cycles, while pursuing the ideal case, i.e. complete fault localization (see the definition in the beginning of section 3.4). In general, the less average number of elements exists in faulty candidate sets for all alarm codes, the better is the performance of MFLA. To quantitatively measure the grade of fault localization, a new metric, the localization degree (denoted as $I$ ), is defined as the 
average size of all non-empty faulty candidate sets produced by MFLA. Here the size of a countable set is the number its elements [66]. For the results of the fault localization algorithm in a finite undirected graph, each faulty candidate set is countable and finite. The size of a faulty candidate set is the number of faulty candidate elements (links) in it.

Let $C=\left\{c_{1}, c_{2}, \cdots, c_{M}\right\}$ be a set of $m$-cycles in graph $G$. Since each alarm code consists of $M$ bits, the number of possible alarm codes is $2^{M}-1$. Let $s_{k}$ be the faulty candidate set for alarm code $\mathbf{m}_{k}$, where $k=1,2, \cdots, 2^{M}-1$. Please note for a given AON, some alarm codes are not applicable (see Table 3.3). Let $D$ be the collection of all applicable $s_{k}$. For an alarm code $\mathbf{m}_{k}, F_{i}^{(k)}=0$ indicates that link $e_{i}$ is a faulty candidate for it. Thus the corresponding faulty candidate set $s_{k}$ is not null only if $\bar{F}_{1}^{(k)} \oplus \bar{F}_{2}^{(k)} \oplus \ldots \oplus \bar{F}_{L}^{(k)} \neq 0$. Note that $\bar{\bullet}^{-}$is the complement of a Boolean variable and the addition $\oplus$ is the Boolean operation here. Clearly, we have $\left|s_{k}\right|=\sum_{i=1}^{L} \bar{F}_{i}^{(k)}$ (note that the summation is arithmetic operation, the same hereinafter). Therefore, the localization degree can be calculated as the following,

$$
I=\frac{\sum_{s_{k} \in D}\left|s_{k}\right|}{|D|}
$$

and,

$$
I=\frac{\sum_{k=1}^{2^{M}-1} \sum_{i=1}^{L} \bar{F}_{i}^{(k)}}{\sum_{k=1}^{2^{M}-1}\left[\bar{F}_{1}^{(k)} \oplus \bar{F}_{2}^{(k)} \oplus \cdots \oplus \bar{F}_{L}^{(k)}\right]}
$$

For the complete fault localization, each non-empty candidate set has only one element, thus the corresponding localization degree is $I_{\text {comp }}=1$. In general, some 
candidate sets might have multiple elements thus the localization degree is usually larger than one, i.e., $I \geq 1$. The target of fault localization is to find the minimum set of potential malfunctioned network resources based on the alarms generated in the fault detection phase. Thus, a successful fault localization algorithm must keep the localization degree $I$ in minimum. The higher $I$ implies that more monitors are needed for locating a network fault to a unique link.

\subsubsection{Wavelength overhead}

In the $m$-cycle based FDL approaches, some wavelength channels in each link are reserved for $m$-cycles. These channels cannot be used for carrying user traffic and therefore become an overhead. The number of reserved wavelengths in a link is equivalent to the cover depth of that link in an $m$-cycle set. The cover depth of a link is the number of $m$-cycles in which that link appears. For an edge $e \in E$, let $C(e)$ denote the number of cycles in $C$ that contain $e$, i.e. $C(e)=\left\{i: e \in c_{i}\right\} \mid$. When $C(e)=t$, it is said that the cover depth of edge $e$ is $t$ in $C$.

To quantitatively analyze the relative overhead due to $m$-cycles, two metrics are respectively defined as the following: the maximum and average wavelength overhead brought to the network by $m$-cycles per link,

$$
\begin{aligned}
& W O H_{\max }=\frac{\Lambda_{\max }}{\Delta} \times 100 \% \\
& W O H_{\text {avg }}=\frac{\Lambda_{\text {avg }}}{\Delta} \times 100 \%
\end{aligned}
$$

Where $\Delta$ is the number of total available wavelengths in a link, $\Lambda_{\max }$ and $\Lambda_{a v g}$ are the 
maximum and average numbers of reserved wavelengths in a link, respectively. $\Lambda_{\max }$ represents the worst case of reserved wavelengths in a single link. Here a constant $\Delta$ is applied to all links in an AON but in the real world this is not necessary. In Chapter 6.4.2, more details will be discussed about the impacts on MFDL performance in terms of various numbers of available wavelengths for different links.

The length of a cycle is the number of edges it contains, denoted by $\operatorname{len}\left(c_{i}\right)=\left|c_{i}\right|$. The length of $\mathrm{C}$, denoted as $\operatorname{len}(C)$, is the summary of all cycles' lengths in $C$. Obviously,

$$
\operatorname{len}(C)=\sum_{i=1}^{M}\left|c_{i}\right|=\sum_{j=1}^{L} C\left(e_{j}\right)
$$

where $C\left(e_{j}\right)$ is the cover depth of link $e_{j}$ in cycle cover $C$.

The average number of reserved wavelengths, $\Lambda_{a v g}$, for all edges is equal to the average cover depth and,

$$
\Lambda_{\text {avg }}=\frac{\sum_{i=1}^{L} C\left(e_{i}\right)}{L}=\frac{\operatorname{len}(C)}{L}
$$

thus,

$$
W O H_{\text {avg }}=\frac{\operatorname{len}(C)}{L \Delta} \times 100 \%
$$

According to formulae 3.5.7, it needs to minimize the cycle cover length in $m$-cycle construction to minimize the average wavelength overhead. Consequently, $m$-cycle construction can also be formulated to the least cost cycle cover problem (CCP) for unweighted graphs.

With the deployment of DWDM technology, the number of wavelengths in a single link tends to become larger and larger. For example, it was reported even in 2001 that 
432 wavelengths could be multiplexed into a single fibre [57]. In current commercial DWDM systems, the number of available wavelengths within a fibre is relatively high [67]. More importantly, the supervisory channel requires only the minimum of bandwidth, i.e., higher bandwidth channels (e.g. OC-192) are applied for user data carrying and the lowest bandwidth channel for $m$-cycle. In this way, the cost of wavelength in MFDL could be further lower. Therefore, it is safely to say that, relatively, $\Lambda_{\text {avg }} \ll \Delta$ and $\Lambda_{\max } \ll \Delta$, as well conservatively to set $\Delta=64$ to calculate $W O H_{a v g}$ and $\mathrm{WOH}_{\max }$.

\subsubsection{Cost reduction}

The cost of the proposed fault detection and localization approach mainly depends on the cost of those optical network monitors for monitoring and the cost for wavelength channels in fibre links. With the DWDM technology, the cost of wavelength channels is becoming much cheaper than the cost of network monitors like optical transceivers and OSAs [67-68]. Comparing to the traditional monitor-per-link fault detection approach, the proposed $m$-cycle based approach trades off the cost of monitors with the cost of wavelengths in dark fibres and therefore can achieve a significant cost reduction, especially for network service carriers where spare wavelength are already there.

Since a monitor and a dedicated supervisory channel are assigned for each $m$-cycle, the cardinality (cycle number) of the $m$-cycle set, $|C|=M$, represents the total required monitors. Thus, it is a measurement of the cost for the $m$-cycle based FDL approaches. To evaluate the performance of the approach in terms of costs, the number of required monitors (equivalent to the number of $m$-cycles, $M$ ) in the proposal is compared with that 
using one monitor per link (i.e. the number of required monitors equals to the number of links, $L$, in the given graph). A relative cost reduction in terms of the required monitors is defined for the proposal,

$$
G_{n}=\frac{L-M}{L} \times 100 \%
$$

It should be noted that the localization degrees are typically different in the proposal compared to the monitor-per-link case. A fair comparison can be made only if they achieve the same degrees of localization. Because the monitor-per-link approach can always achieve complete fault localization while the $m$-cycle based approach typically cannot, some extra monitors are added for those links that cannot be fully localized under the MFDL approach so that complete fault localizations are also achieved. For example, a straightforward method would be the following one. If there are $K>1$ links in an MFLA candidate set, then there are $K-1$ extra monitors assigned to $K-1$ of those $K$ links. Some methods that are more efficient might be applied for achieving complete fault localization, e.g. using extra $m$-cycles. Denote the number of all extra monitors as $M^{\prime}$. Then the complete fault localization can be achieved and the calculation of cost reduction is slightly revised from 3.5.4 to include $M^{\prime}$,

$$
G_{n}^{\prime}=\frac{L-\left(M+M^{\prime}\right)}{L} \times 100 \%
$$

Clearly, if $M=L$ or $M+M^{\prime}=L$, then the cost reduction will be zero. Further, if $M>L$ or $M+M^{\prime}>L$, then the MFDL will be more expensive than the monitor-per-link scheme. Therefore, one of the most important objectives in m-cycle construction is to minimize $M$ and guarantee $M$ is much less than $L$. 


\section{Chapter 4}

\section{$M$-Cycle Construction Algorithms}

$\mathrm{T}$ has been proven that cycle covers exist for each bridgeless, connected, undirected
graph and cycle construction algorithms in running time $o\left(n^{2}\right)$ exist in theory, while $n$ is the number of nodes in the graph [69]. A polynomial-time algorithm was really developed and evaluated for cycle cover construction in [70]. Such works were focused on the least-cost cycle cover problem but did not consider the localization degree and the cycle number (equivalent to the cost of monitoring devices). According to its definition, the $m$-cycle construction is a cycle cover problem (CCP). To find a cycle cover for a given network topology, with the consideration of cycle number and cycle length, the CCP is introduced in this chapter and existing algorithms for solving CCP are briefly surveyed. The bounds of both cycle number and cycle length of the CCP solutions are also discussed. After the survey, two $m$-cycle construction algorithms are developed: the depth-first searching (DFS) algorithm [71] improved with some heuristic rules (called HDFS) and the Shortest-Path Eulerian Matching (SPEM) algorithm simplified from the minimum weight Eulerian matching algorithm [71]. The two algorithms are applied to four typical network examples obtained from the real world to evaluate their performance in terms of the metrics defined in Chapter 3.5. 


\subsection{Introduction to Cycle Cover Problem (CCP)}

Let $G(V, E)$ be a connected undirected graph where $V=\left\{v_{1}, v_{2}, \cdots, v_{n}\right\}$ is the node (vertex) set and $E=\left\{\left(v_{i}, v_{j}\right) \mid v_{i}, v_{j} \in V, i<j\right\}$ is the link (edge) set. The cycle cover problem is to find a least cost set of simple cycles whose union contains all links and nodes in the graph at least once. A simple cycle is a cycle that contains no repeated vertices except for the starting and ending vertices. Many important practical problems, such as routing, navigation, electric circuit analysis, and irrigation system design, can be modeled as cycle cover problems (CCP) with specific constraints on the cycles in the solution. The cycle cover problem has been extensively studied since it first arose in the design of irrigation systems in 1936 [84]. The book [63] reviewed much of such literatures. The CCP is also referred as the shortest cycle cover problem or the minimumcost cycle cover problem (MCCP). It is referred as the CCP hereafter.

Usually some constraints are put on the cycles while solving the $\mathrm{CCP}$. The most common constraint to the cycles in a solution of CCP is the cycle length. The general $\mathrm{CCP}$ with the cycle length constraint is defined as the Constrained Cycle Cover Problem (CCCP), or called the Bounded Cycle Cover Problem (BCCP). In this thesis it is hereafter referred as CCCP.

As an interesting application of CCP, Chapter 3 introduced the $m$-cycle based fault detection and localization (MFDL) scheme for mesh AONs, which was published in [72, $74,76,85]$. In this scheme, a cycle cover is constructed for the mesh AON. A monitor and a supervisory optical channel are assigned to each cycle in the cover. A network fault 
triggers alarms in the cycles that contain the faulty source, and thus it can be located to the common part of those cycles.

In MFDL scheme, if a link is covered by $t$ cycles, then $t$ wavelengths are reserved in this link for supervisory channels that cannot be used for carrying user traffic. The total cost of all reserved wavelengths is the cover cost if each link-cost is set as the wavelength-cost within this link (the wavelength-cost might be different for various links). For the case that the wavelength-cost is constant for all links, the network can be modeled as an un-weighted graph and the cost of reserved wavelengths can be measured by the cover cost (equivalently called cover length). The second cost of the MFDL scheme is the network monitors assigned to all $m$-cycles, which can be measured by the number of cycles in a cover. Therefore, it is necessary to take account of both the cover length and cycle number while building a cover for MFDL.

\subsubsection{Bound of the cover length}

Itai and Rodeh conjectured that it is NP-complete for minimizing the cost of the cycle cover for a given graph [69]. Later on, the minimum-cost cycle cover problem was proven to be NP-hard [86]. Therefore, researchers developed some algorithms to quickly find a suboptimal solution of CCP that is within a certain range of the optimal one, instead of finding the optimal solution. Many efforts have been focused on improving the range of feasible solutions closer to the optimal one, e.g., the study of the Chinese postman problem.

The Chinese postman problem (CPP), which was first introduced in [87], is a wellknown relaxation of CCP. The CPP is to determine the least cost traversal of a given 
graph. Any solution of the CPP can be decomposed into a set of cycles that cover all links in the graph. More importantly, low order polynomial algorithms exist for CPP [88]. However, some of these cycles are of the form $\left(v_{i}, v_{j}, v_{i}\right)$ and not allowed in the solution of CCP. It has been shown that the cost of a shortest cycle cover is larger than that of a CPP solution [69]. For graph $G(V, E)$, let $c c(G)$ and $c p(G)$ be the cover cost of the solution for CCP and CPP respectively, then $c p(G) \leq c c(G)$ for connected graphs [69], thus the CPP solution provides a lower bound on the value of the optimal CCP solution. It is also known that $\operatorname{co}(G) \leq|E|+|V|-1$, where $|\bullet|$ is the cardinality of a set. Although it has never been proven, researchers commonly conjecture $c c(G) \leq|E|+|V|-1$ in bridgeless graphs [69]. Table 4.1 shows the upper bound of the optimal solutions for CCP has been improved along the time to approach the conjecture. Such bounds are important for evaluating the quality of feasible solutions of CCP.

\subsubsection{Bound of the cycle number in the cover}

The number of cycles in the solution is another consideration for solving the CCP for cycle-based fault detection and localization. The cycle cover given in [69] uses $1+\lfloor\log |V|\rfloor$ cycles. The number of cycles is dramatically reduced to four in its following work [89]. The covers given in [90] and [91] also require four cycles. Reference [92] proved that there always exists a cover consisting of three cycles. The bound of cost for CCP solutions was improved in a series of papers, but the authors did not give the cycle number to reach the bound $[70,93-94]$. These results about the cycle number in the cover are summarized in Table 4.1. Notice that these cycle numbers are for CCP without constraints of cycle length, cycle number, and complete fault localization. 
Table 4.1 Bounds of CCP suboptimal solutions

Literature

Itai-1978 [69]

Itai-1981 [89]

Bermond-1983 [90]

Alon-1985 [91]

Fraisse-1985 [92]

Fan-1992 [70]

Fan-1997-1 [93]

Fan-1997-2 [94]
Bound of CCP solution

$$
\mathcal{c c}(G) \leq|E|+2(\log |V|)|V|
$$

$$
c c(G) \leq|E|+6|V|-7
$$

4

Cycle number

\section{Complexity}

$$
c c(G) \leq|E|+\frac{7}{3}(|V|-1)
$$

4

$$
c c(G) \leq|E|+\frac{7}{3}(|V|-1)
$$

4

$$
O\left(|V|^{2}\right)
$$

$$
c c(G) \leq|E|+\frac{5}{4}(|V|-1)
$$

$$
c c(G) \leq|E|+\frac{6}{5}(|V|-1) \quad \text { N/A }
$$

N/A

$$
O\left(|E|+|V|^{2}\right)
$$

$O\left(|E|+|V|^{2}\right)$

$O\left(|E|+|V|^{2}\right)$

$O(|E| \cdot|V|)$

$c c(G) \leq|E|+\frac{11}{10}(|V|-1) \quad$ N/A $\quad$ N/A

$c c(G) \leq|E|+\frac{25}{24}(|V|-1) \quad$ N/A

N/A

\subsection{Existing Algorithms for Solving CCP}

The CCP has been proven to be NP-hard [69]. The class of NP-hard problems can be understood as the class of problems that are NP-complete or harder. At present, to solve an NP-complete problem for any nontrivial problem size, one of the following approaches is used:

- Approximation: An algorithm that quickly finds a suboptimal solution that is within a certain range of the optimal one. 
- Probabilistic: An algorithm that provably yields good average runtime behavior for a given distribution of the problem instances - ideally, one that assigns lower probability to inputs that occur more rarely.

- Special cases: An algorithm that is provably fast if the problem instances belong to a certain special case. Fixed-parameter algorithms can be seen as an implementation of this approach.

- Heuristic: An algorithm that works "reasonably well" on many cases, but for which there is no proof that it is always fast (a rule of thumb, intuition).

Therefore, CCP is usually solved using the above algorithms for NP-complete problems. Most common methods for solving CCP are heuristic algorithms. The CCP can be also considered as an optimization problem that chooses an optimal subset of cycles from a set of candidate cycles (or a set of all cycles) in a given graph. Consequently, some standard optimization methods, such as the Integer Linear Programming (ILP) and Branch-and-Bound (B\&B) searching are also applicable [77-78]. Some other optimization algorithms like evolutionary algorithms or genetic algorithms (including Pareto's approach for multi-objective optimization) might not be applicable due to the following facts:

- Optimal solutions are not guaranteed using evolutionary algorithm

- Although the worst-case time complexity of B\&B is the same as the complexity of exhaustive search, which is exponential, in real-life test cases, it is proven to speed up the search considerably, e.g., B\&B improves the search up to $90 \%$ for some problems [134]. 
- Complexity of evolutionary algorithms has no lower-bound (worst case), worse than $\mathbf{B} \& \mathrm{~B}$

Some reported cycle cover construction algorithms are chronologically listed below.

\section{Itai-1978 [69]}

Itai proposed a probabilistic cycle cover construction algorithm containing some random choices in this paper. The algorithm firstly finds a spanning-tree and constructs a cycle for each edge that is not in the tree. Then it removes a cycle if all edges in this cycle are covered by other cycles. After checking all cycles generated in the first step, the algorithm output a cover with the length no more than $|E|+2(\log |V|)|V|$. The average computational complexity of the spanning-tree based probabilistic algorithm is $O\left(|V|^{2}\right)$, although this algorithm may not terminate in theory (with the probability of almost null).

\section{Itai-1981[89]}

An improved heuristic algorithm was developed in [89] for 2-edge-connected graphs. This algorithm firstly finds three spanning-trees and constructs an Eulerian sub-graph for each spanning-tree. A graph or sub-graph is Eulerian if it has at least one Euler circuit. An Euler circuit is a path that starts from and ends to the same node, and contains all links in the graph or sub-graph. In this heuristic algorithm, each Eulerian sub-graph is decomposed into a set of cycles. The union of cycles generated from the three Eulerian sub-graphs is a feasible solution for the CCP. The length of the solution obtained by this algorithm is no more than $c c(G) \leq|E|+6|V|-7$ and the corresponding computational 
complexity is $O\left(|E|+|V|^{2}\right)$.

\section{Chickering-1995[95]}

This reference analyzed an interesting exponential greedy algorithm for finding the shortest basis. Each cycle can be represented by a vector whose dimension is the number of links in the graph and the value of each element is binary. The element at the position corresponding to link $e$ has a value of one when the cycle covers $e$, and zero otherwise. All cycles not in the basis of the graph can be represented as a linear combination of the cycles in the basis, while the linear combination of cycles is the exclusive OR operation among corresponding elements of those cycle's representative vectors. This algorithm compares the length of each cycle with that of the linear combination of the rest cycles in a given cycle basis, and replaces this cycle with the linear combination of the others if the later has a shorter length. Such comparison is executed till no more cycle can be replaced. The algorithm is based on the fact that any linear combination of a group of cycles in a graph is still a cycle. A cycle basis for a graph is a set of cycles such that any cycle in the given graph can be represented by a linear combination of the cycles in the basis. The output of this algorithm is a shortest cycle basis but not necessarily to be the shortest cycle cover. Nevertheless, it is possible to be applied to CCP and might find a new direction for developing CCP algorithms.

\section{Labbe-1998 [96]}

The algorithm reported in [96] firstly augments the given graph to be Eulerian using the least-cost matching (equivalent to the shortest-path Eulerian matching, will be explained in details in Chapter 4.4), then finds an Eulerian tour and decompose it by the 
end-pairing algorithm described in [88]. A cost coefficient is assigned to each edge for the calculation of edge and cycle cost. If a cycle is of the form $\left(v_{i}, v_{j}, v_{i}\right)$, it is defined as an illegal cycle. For an illegal cycle the algorithm replaces edge $\left(v_{i}, v_{j}\right)$ by the shortest chain between nodes $v_{i}$ and $v_{j}$. While choosing the successive cycles, the authors analyzed a set of heuristics:

1) Random choice.

2) The cycle covers the least-cost edge among the edge not covered in previous cycles.

3) Largest-cost edge.

4) Least-cost edge not forming an illegal cycle.

5) Largest-cost edge not forming an illegal cycle.

6) Edge $\left(v, v_{j}\right)$ with the largest number of uncovered edges incident to $v_{j}$.

The random choice was considered for benchmarking. The authors claimed that all heuristics succeed in producing optimal or near-optimal solutions within reasonable computing time. The analysis was based on empirical performance, rather than worstcase results.

\section{Immorlica-2005 [97]}

This latest reference reported a (heuristic) greedy algorithm for solving CCP with a limit of cycle length (the cycle length is the number of links in the cycle). This algorithm greedily selects the least-cost feasible cycle and iterates until all edges are covered by a cycle in the solution set. The algorithm is described as below ( $\mathrm{L}$ is initialized as the set containing all links in a given graph): 
- While there is an edge in $\mathrm{L}$, do the following

- Find the least-cost cycle $\mathrm{C}$ in the graph consisting of at most $\mathrm{k}$ edges. If there is more than one such cycle, pick one arbitrarily.

- Add $\mathrm{C}$ to the solution and remove its edges from $\mathrm{L}$.

A series of improvements, e.g., dual fitting and approximation, were applied in this paper to improve the performance of this heuristic algorithm. Details were thoroughly analyzed in the reference.

In summary, many algorithms have been proposed for solving $\mathrm{CCP}$ but most of them are heuristic based and output near-optimal solutions instead of the optimal solution. The above algorithms fall into four categories: tree-based, Eulerian matching, greedy, and cycle-basis based algorithms. Empirical performance analysis showed that all of them could achieve a good feasible solution within reasonable time for the given CCP. In this Chapter, two cycle-cover construction algorithms: HDFS and SPEM algorithms are proposed due to their simplicity and effectiveness. Some heuristic rules, e.g., the largest/smallest node choice rule (will be explained in the description of HDFS algorithm) and the shortest-path Eulerian matching (details in the descrition of SPEM algorithm), are incorporated to their original depth-first searching and Eulerian matching respectively. With such heuristics, the low localization degree can be achieved in the results of MFLA algorithm with $m$-cycles obtained from HDFS and SPEM. 


\subsection{Heuristic Depth-First Searching (HDFS) Algorithm}

Given a connected, bridgeless graph $G(V, E)$ (note that all graphs discussed in this thesis satisfy the constraint given at the end of Section 3.2), starting from any node $n \in V$, all links in $E$ can be traversed by depth first searching (DFS). Let the traversed part of $G$ be $G^{\prime}\left(V^{\prime}, E^{\prime}\right)$ during the DFS and this is the current DFS path. Let node $x$ be the last node of the current DFS path. For the next link to be traversed, for example link $e$ from node $x$ to node $y$, it defines that the current node is $x$ and the traverse direction is from node $x$ to $y$. Nodes $x$ and $y$ are called the in- and out-end of link $e(x, y)$ respectively. If $y \in V^{\prime}$, there must exist a path $p(y, \cdots, x) \in G^{\prime}$, i.e. a part of the current DFS path. Then path $p(y, \cdots, x)$ and link $e(x, y)$ form a cycle. The heuristic depth first searching (HDFS) algorithm [72] is developed as below for finding a cycle cover (a descriptive example of HDFS application will be given later in this sub-chapter),

1) Initial: Given a graph $G(V, E)$, let the cycle cover $C=n u l l$; number all nodes in $V$; and label all nodes in $V$ and all links in $E$ as "uncovered".

2) Set the current DFS path to be null. Randomly select an uncovered node as the origin of the DFS. Set the label of the current node to be "covered". If all nodes are "covered", randomly select the node that is incident to at least one uncovered link;

3) From the current node, select a link incident to it (the in-end of the link is the current node) and append the selected link to the current DFS path, according to the following priority. If multiple links are available, alternatively set the heuristic 
node choice rule to be the largest/smallest-node-first, i.e., if at the current iteration it chooses the link with the out-end with the largest node number, then in the next iteration it will choose the link with the out-end with the smallest node number.

1st) Select the link whose out-end is the origin of the current DFS and stop the current DFS iteration;

2nd) If the origin node is not reachable at this step, select an uncovered link such that the out-end of the selected link is also uncovered. If there are multiple such links available, apply the current node choice heuristic rule;

3rd) Select an uncovered link that has a covered out-end, also apply the current node choice heuristic rule;

4th) Select a covered link whose out-end is also covered by applying the current node choice heuristic rule.

4) Repeat step 3) until the current DFS returns to the origin, then a cycle $c$ is formed and added to the cover $C$. Stop the current DFS and label all the links and nodes in cycle $c$ as "covered";

5) Repeat 2)-4) until all nodes and links in $G(V, E)$ are covered. Then the cycle cover set $C$ is the required $m$-cycle cover.

In existing DFS algorithms, the random choice rule is primarily applied for choosing the next node to be traversed. Such node selection may lead to repeatedly choose a certain node subset and thus unnecessarily increase the cover depth for some links. In the above HDFS algorithm, all nodes (in-ends and out-ends, which are defined at the beginning of Chapter 4.3 ) are numbered. While choosing the next node to be traversed in the DFS iteration, the largest/smallest-node-first heuristic rules are applied whenever 
multiple candidates are available for a selection. The heuristic is that, if the algorithm selects the link whose out-end has the largest number among all candidates in the current (outer) iteration of the algorithm, then it will select the link whose out-end has the smallest number among all candidates in the next (outer) iteration of the algorithm. Such a heuristic avoids selecting a link repeatedly in consecutive iterations of the algorithm and thus it distributes cycles evenly in the graph. Therefore, this heuristic rule avoids covering a link with many different cycles in the cover, i.e., avoids reserving large number of wavelengths in a link for monitoring. Additionally, uncovered links are always selected with higher priority than covered links in the algorithm (see step 2 and 3). This heuristic rule keeps the number of cycles small in the final cover set, i.e. keeps the number of required monitors small and thus keeps the cost small.

As a descriptive example, the above algorithm (HDFS) is applied to the network example given in Figure 3.1. As shown in Figure 4.1, the nodes are numbered from 1 to 10 before starting the DFS. All nodes and links are labeled as "uncovered" and the cycle cover is set to null. During the DFS, the following iterations are executed,

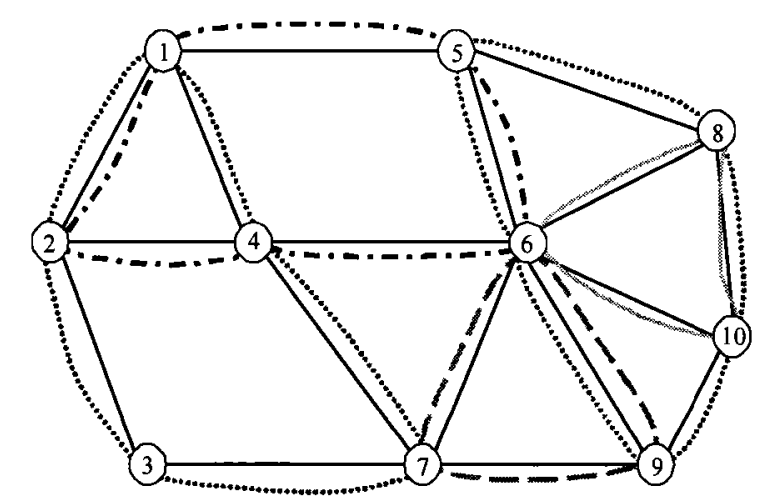

Figure 4.1 The cycle cover obtained by HDFS for the network example in Figure 3.1 
First DFS: Select node 1 as the DFS origin. From node 1 there are 3 uncovered links whose out-ends are also uncovered: $(1,2),(1,4)$, and $(1,5)$ available for the next step of the current DFS traverse. Applying the smallest-node-first rule, the algorithm selects link $(1,2)$. Repeat such selections until node 1 is visited again. Then cycle $1-2-3-7-4-1$ is obtained and added to $C$. Nodes in set $\{1,2,3,7,4\}$ and links in set $\{(1,2),(2,3),(3,7)$, $(7,4),(4,1)\}$ are labeled as covered.

Second DFS: Select node 5 as the DFS origin this time. From node 5 there are 3 uncovered links that could be selected: $(5,1),(5,6)$ and $(5,8)$. Since node 6 and 8 are uncovered, link $(5,6)$ and $(5,8)$ are prior to $(5,1)$. Alternatively to the first DFS, the largest-node-first rule is applied and select link $(5,8)$. After this DFS the cycle 5-8-10-9$6-5$ is obtained and added to $C$. Nodes in set $\{5,8,10,9,6\}$ and links in set $\{(5,8),(8,10)$, $(10,9),(9,6),(6,5)\}$ are labeled as “covered".

Similarly, cycles $6-4-2-1-5-6,8-6-10-8$ and 6-7-9-6 are added to $C$ in the remainder iterations. After 5 iterations, all links in the graph are covered and a cover with 5 cycles is obtained as depicted in Figure 3.1.1. These are cycles that would be used as $m$-cycles.

\subsection{The Shortest-Path Eulerian Matching (SPEM)}

\section{Algorithm}

As defined in Chapter 4.2, a circuit containing all the links in a graph $G(V, E)$ is said to be an Euler circuit of $G$, where a circuit is a path that starts from a node and ends to the 
same one (some nodes might be visited more than once). A graph is Eulerian if it has at least one Euler circuit. For a Eulerian graph, the Euler circuit covers all links exactly once. If we traverse the Euler circuit by following links in it until a node is re-visited, the traversed part forms a sub-cycle. Then we can remove this part from the Euler circuit and traverse the remainder part until all links are removed [71]. In this way, the Euler circuit is decomposed into sub-cycles and all these sub-cycles constitute a cycle cover $C$ for the given Eulerian graph. Due to the fact that no two cycles in $C$ have a common link, each link in the graph appears in only one cycle. Clearly, this approach constructs a cycle cover that requires only one wavelength channel per link for monitoring (in AONs whose topologies are Eulerian) and therefore generates the minimum wavelength overhead and monitor cost.

Euler proved that a graph $G(V, E)$ is Eulerian if and only if every node in $V$ has an even degree [73]. Thus a non-Eulerian graph has some nodes with odd degrees. Since each link connects two nodes, the total number of odd-degree nodes must be even. We can augment a given non-Eulerian graph by adding virtual links connecting pairs of odddegree nodes to construct an Eulerian graph, the so-called Eulerian matching. After a graph is augmented to be Eulerian, we can then find its Euler circuit and decompose this Euler circuit into a cycle cover. This cycle cover will traverse each link/virtual link once. However, each virtual link has to be implemented by a path that travels through some real links. Thus the physical cycle cover may traverse a link more than once. To minimize the average link cover depth, the path that implements a virtual link should be as short as possible, i.e. the shortest path between odd-degree node pairs. The existing shortest-path matching algorithm reported in [74] is simplified and based on that a slightly improved 
(simplified) Shortest-Path Eulerian Matching (SPEM) algorithm is described below,

1) For a graph $G(V, E)$, if $G$ is Eulerian, go to step 4) directly; if $G$ is non-Eulerian graph, find the set $V^{\prime}$ of odd-degree nodes;

2) Let the matching set of path, $G_{m}$, be null;

3) In $V^{\prime}$, start from an arbitrary node $x$ and find the shortest path to every other node, select the smallest one among them, denote as $p(x, y)$. Add the path $p(x, y)$ to $G_{m}$ (now some node pairs in $G$ have multiple edges) and remove $x, y$ from $V^{\prime}$

4) Repeat (2)-(3) until $V^{\prime}=$ null . Now the algorithm obtains a Eulerian matching that starts from node $x$. Find such matching path sets starting from other nodes in $V^{\prime}$ and select a matching set with the shortest path length as the shortest-path Eulerian matching and add the paths in it to graph $G(V, E)$. Now $G$ is Eulerian;

5) Find an Euler circuit of the augmented $G(V, E)$ and decompose it into subcycles. These sub-cycles are the required $m$-cycles for the original graph.

The algorithm is demonstrated here by applying it to the network example shown in Figure 3.1. In this graph, the algorithm firstly labels the degrees for all nodes (see Figure 4.2). The odd-degree node set is $\{1,2,5,8,9,10\}$. For node 1 , the shortest path to another node is $(1,5)$ or $(1,2)$, which are a single hop. Select $(1,2)$ and remove nodes 1 and 2 from the odd-degree node set. Repeatedly the matching path set $\{1-2,5-8,9-10\}$ (total length is 3 ) is obtained. For the existing tie, if $(1,5)$ is selected at the first step, the matching path set would be $\{1-5,2-3-7-9,8-10\}$. The total length is five, larger than the first matching path set and thus is dropped. By enumerating all possible pairs in this way, the shortest 
path matching can be reached (links in bold lines in Figure 4.2). The node degrees changed by the matching paths are labeled in the brackets in Figure 4.2. Now all node degrees are even and the augmented graph is Eulerian.

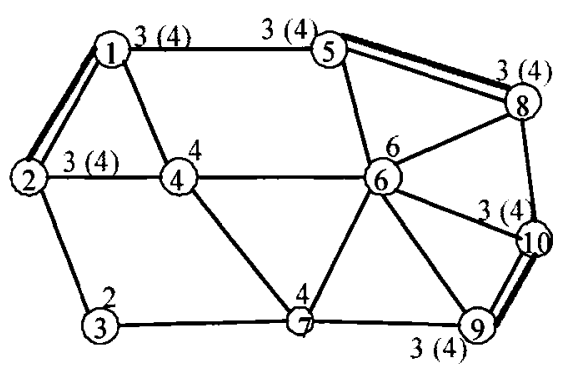

(a)

\section{A Eulerian circuit: \\ $1-2-3-7-4-2-1-4-6-5-8-6-7-9-6-10-9-10-8-5-1$}

(b)

Figure 4.2 Demo of the SPEM algorithm: (a) The shortest path Eulerian matching;

(b) An Euler circuit

An Euler circuit can be found by any existing traversing algorithms, e.g., the standard DFS algorithm. In this example an Euler circuit is obtained by the DFS algorithm reported in [58] and is shown in Figure 4.3. According to the SPEM algorithm described above, the Eulerian circuit is then decomposed into sub-cycles, i.e. required $m$-cycles. The decomposition rule is as the below: following the original Eulerian circuit and traversing the circuit node by node. Once the algorithm re-visits a node that have already visited before, the traversed sub-path between the two visits is a cycle and be added to the required $m$-cycle set. Please note that a two-edge cycle, e.g., 10-9-10, is not considered as a "real" cycle and thus is avoided. After generate a cycle, remove this part from the original Eulerian circuit, i.e., it is shrunk to a single node in the original Eulerian circuit. Repeat this process until all nodes in the original Eulerian circuit are removed. As a demonstration, the decomposition process for the network example with the shortest-path 
matching in Figure 4.2 is showed in Figure 4.3.

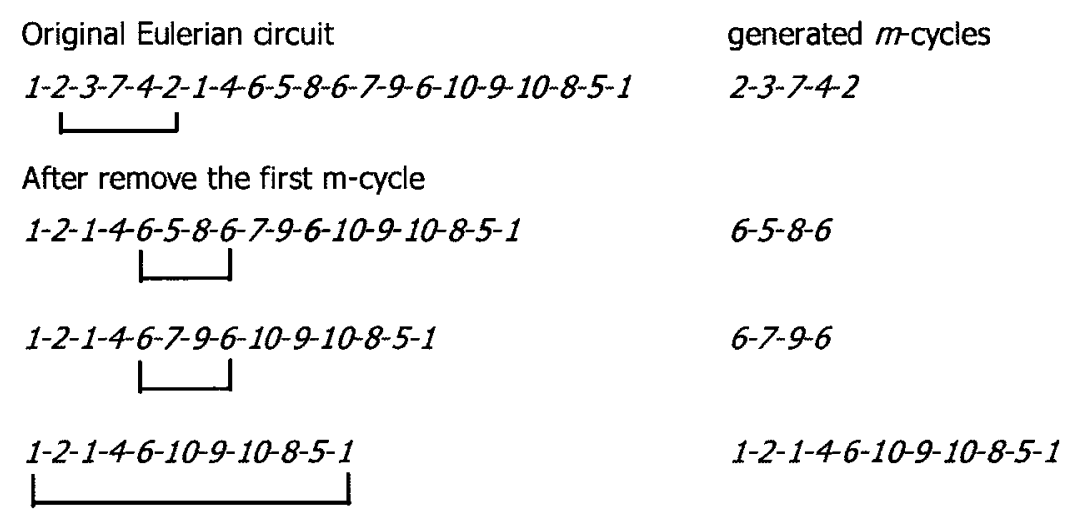

Figure 4.3 Eulerian circuit decompositions

\subsection{Examples and Performance Evaluation}

To evaluate the performances of the $m$-cycle construction algorithms developed in this chapter, algorithms are applied together with the MFLA (see Chapter 3.3) to four typical example networks (NSFNET, ARPA2, SmallNet, and Bellcore). These network examples are from the real world, popular and applied to most reported research works in this area [3]. The topologies of them are shown in Figure 4.4. The $m$-cycles obtained by the HDFS and SPEM $m$-cycle construction algorithms in the four networks are listed in Figures A.1 and A.2 respectively in appendix A of this thesis, including the corresponding shortestpath Eulerian matching of SPEM algorithm. With the obtained $m$-cycles, the MFLA is applied to those network topologies and list all possible alarm codes with corresponding fault localization results from Tables A.1 - A.8 in appendix. In this section, performances 
of both $m$-cycle construction algorithms and the fault localization algorithm, MFLA, are evaluated in terms of those metrics described in Chapter 3.5, including time-complexity of both algorithms, the localization degree of MFLA, wavelength overhead due to $m$ cycles, and cost reduction over the monitor-per-link method.

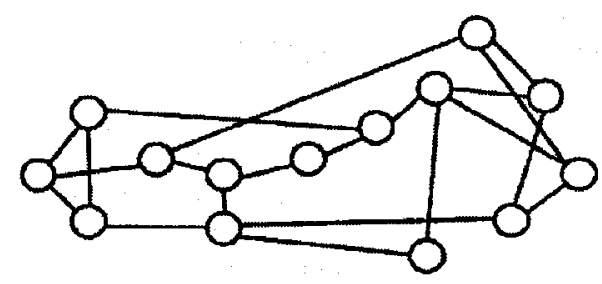

(a) NSFNET: 14 nodes, 21 links

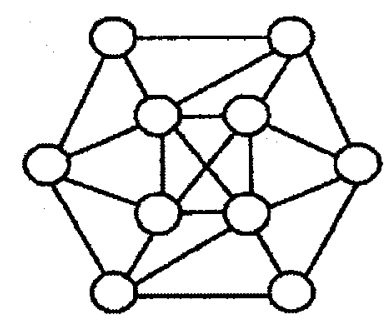

(c) SmallNet: 10 nodes, 22 links

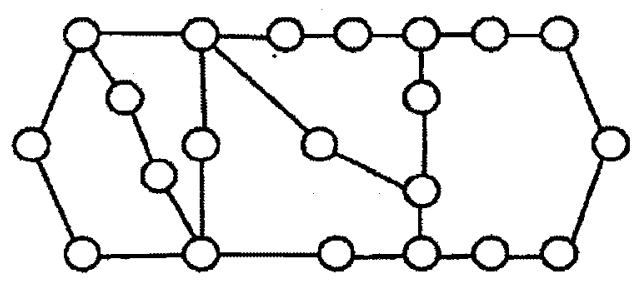

(b) ARPA2: 21 nodes, 25 links

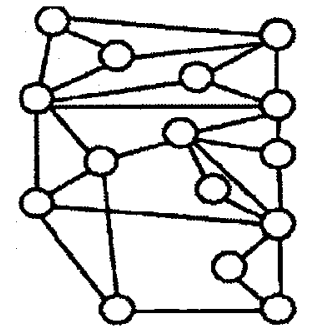

(d) Bellcore: 15 nodes, 28 links

Figure 4.4 Typical example networks

(a) NSFNET, 14 nodes and 21 links; ARPA2, 21 nodes and 25 links;

(b) SmallNet, 10 nodes and 22 links; Bellcore, 15 nodes and 28 links.

\subsubsection{Localization degree}

By enumerating the faulty candidate sets for all possible alarm codes in an AON with $m$-cycles, the localization degrees can be immediately calculated based on the results listed in Tables A.1-8 (see appendix A) for HDFS and SPEM algorithms respectively, according to equation 3.5.1. Here the localization results are based on the single-failure scenario for simplicity, but they could be easily extended to multiple-failure scenarios by 
including the alarm combinations in the tables for multiple-failure scenarios, as described in the end of Chapter 3.3.

Table 4.2 Comparison of MFLA results for HDFS and SPEM

\begin{tabular}{|c|c|c|c|c|c|c|}
\hline Network & Algorithm & $N$ & $L$ & $\begin{array}{c}\text { avg. node } \\
\text { degree }\end{array}$ & $\begin{array}{l}\text { localization } \\
\text { degree }\end{array}$ & $\begin{array}{c}\text { max candidate } \\
\text { set size }\end{array}$ \\
\hline \multirow{2}{*}{ NSFNET } & HDFS & \multirow{2}{*}{14} & \multirow{2}{*}{21} & \multirow{2}{*}{3.00} & 1.500 & 3 \\
\hline & SPEM & & & & 3.000 & 7 \\
\hline \multirow{2}{*}{ ARPA2 } & HDFS & \multirow{2}{*}{21} & \multirow{2}{*}{25} & \multirow{2}{*}{2.38} & 3.130 & 6 \\
\hline & SPEM & & & & 5.000 & 8 \\
\hline \multirow{2}{*}{ SmallNet } & HDFS & \multirow{2}{*}{10} & \multirow{2}{*}{22} & \multirow{2}{*}{4.40} & 1.470 & 3 \\
\hline & SPEM & & & & 3.670 & 6 \\
\hline \multirow{2}{*}{ Bellcore } & HDFS & \multirow{2}{*}{15} & \multirow{2}{*}{28} & \multirow{2}{*}{3.73} & 2.150 & 6 \\
\hline & SPEM & & & & 4.670 & 8 \\
\hline
\end{tabular}

Table 4.2 summarizes those localization results and compares the localization degree in example networks with $m$-cycles respectively obtained by the HDFS and SPEM algorithms reported in Chapters 4.3 and 4.4. The maximum candidate set size is an indication of the worst-case alarm code in fault localization. The comparison shows that the HDFS algorithm has the better performance than SPEM algorithm in terms of the fault localization degree. Also, the HDFS algorithm always produces less candidate faulty source in the worst case for all network examples, i.e., has much smaller maximum candidate size. The payment for the benefit of the HDFS algorithm in terms of localization degree relies on the higher wavelength overhead and the larger cycle number, 
which will be discussed in the following sub-chapters.

\subsubsection{Wavelength overhead}

The cost of the proposal is measured by the number of required monitors and reserved wavelengths for $m$-cycles. The cost of wavelength channels is evaluated by the reserved wavelengths in links for $m$-cycles, i.e. wavelength overhead defined in Chapter 3.5. The numbers of maximum and average reserved wavelengths in links are summarized in Table 4.3. It shows that numbers of both maximum and average reserved wavelengths for $m$-cycles obtained by HDFS algorithm are slightly larger than SPEM. This is the payment for the benefit in localization degree for this $m$-cycle construction algorithm. The SPEM algorithm has smaller number of reserved wavelengths and thus introduces less wavelength overhead than the HDFS algorithm. As discussed at the beginning of Chapter 2, the number of wavelengths in a single link tends to become larger and larger, and the number of available wavelengths within a single fibre could be considered much larger than the number of reserved wavelengths for $m$-cycles [67]. A conservative small number of available wavelengths per link, $\Delta=64$, is chosen to normalize the wavelength overheads for m-cycle construction algorithms. With such setting, for all example networks the average wavelength overhead is around $2 \%$. For the worst case with HDFS algorithm, the wavelength overheads are still small (4.69\%, see Table 4.3$)$. Such overheads have trivial impact on network utilization. 
Table 4.3 Comparison of wavelength overhead for HDFS and SPEM

\begin{tabular}{|c|c|c|c|c|c|c|c|c|}
\hline Network & Algorithm & $\boldsymbol{N}$ & $L$ & $\begin{array}{c}\text { avg. node } \\
\text { degree }\end{array}$ & $\Lambda_{\text {avg }}$ & $\Lambda_{\max }$ & $\begin{array}{c}W O H_{\text {avg }} \\
\mathbf{( \% )}\end{array}$ & $\begin{array}{c}W O H_{\max } \\
(\%)\end{array}$ \\
\hline \multirow{2}{*}{ NSFNET } & HDFS & \multirow{2}{*}{14} & \multirow{2}{*}{21} & \multirow{2}{*}{3.00} & 1.57 & 3 & 2.45 & 4.69 \\
\hline & SPEM & & & & 1.24 & 2 & 1.94 & 3.12 \\
\hline \multirow{2}{*}{ ARPA2 } & HDFS & \multirow{2}{*}{21} & \multirow{2}{*}{25} & \multirow{2}{*}{2.38} & 1.36 & 3 & 2.12 & 4.69 \\
\hline & SPEM & & & & 1.20 & 2 & 1.87 & 3.12 \\
\hline \multirow{2}{*}{ SmallNet } & HDFS & \multirow{2}{*}{10} & \multirow{2}{*}{22} & \multirow{2}{*}{4.40} & 1.55 & 3 & 2.42 & 4.69 \\
\hline & SPEM & & & & 1.18 & 2 & 1.84 & 3.12 \\
\hline \multirow{2}{*}{ Bellcore } & HDFS & \multirow{2}{*}{15} & \multirow{2}{*}{28} & \multirow{2}{*}{3.73} & 1.43 & 3 & 2.23 & 4.69 \\
\hline & SPEM & & & & 1.14 & 2 & 1.78 & 3.12 \\
\hline
\end{tabular}

Note: $\mathrm{WOH}_{\text {avg }}$ and $\mathrm{WOH}_{\max }$ are in percentage and calculated for $\Delta=64$.

\subsubsection{Cost reduction}

The cost of monitors is weighted by the number of $m$-cycles (denoted as $M$ ), which is equal to the number of required monitors. A cost reduction $\left(G_{n}\right)$ can be calculated as equation 3.5.8 in comparison with the brute force monitor-per-link method. To evaluate the costs for the proposed $m$-cycle based FDL approach, the values of $M$ and the cost reduction is listed in Table 4.4 for all network examples with m-cycles obtained by HDFS and SPEM algorithms, respectively. Clearly, the SPEM algorithm produces smaller number of $m$-cycles and thus has larger cost reduction in the given network examples than HDFS. More specifically, the SPEM algorithm saves more than $80 \%$ of monitors in the monitor-per-link method. For the worst case with the HDFS algorithm, although the $M$ values are larger than SPEM, it still has good cost reduction. The saving percentages for HDFS algorithm are in the range of $60-78 \%$ for network examples. 
As discussed in Chapter 3.5, for a fair comparison with the monitor-per-link method, $M^{\prime}$ extra monitors are added to those links that cannot be fully localized under the MFLA to achieve complete fault localization. Then the cost reduction calculation is revised as equation 3.5.9. Revised cost reduction obtained from the four example networks with $m$-cycles obtained by HDFS and SPEM algorithms are calculated respectively in Table 4.4. Certainly the cost reduction decreases due to the extra monitors. But interestingly, with the supplement monitors, the cost reduction for the HDFS algorithm is better than SPEM algorithm, while the HDFS algorithm also achieves better localization degree. It indicates that the HDFS algorithm seems to be more promising.

For the monitor-per-path method reported in [37], typically each node has to communicate with all other nodes in a $N$-node mesh network, thus the number of potential paths is $N(N-1)$. Even with the $50 \%$ savings of monitors (in maximum) by applying the proposed heuristic monitor placement optimization algorithm [37], the number of required monitors is still $O\left(N^{2}\right)$, while this number in the proposal with $m$ cycle construction algorithms is much smaller than the number of links in the network, i.e., smaller than the number of links in the given network examples. Clearly, the proposed $m$-cycle based approach achieves a significant cost reduction in all examples compared to either the monitor-per-link or the one-monitor-per-path case.

A comparison between the above simulation results and the best results obtainable from monitoring paths might be also interesting. However, due to the obviously difference in terms of optimization onjectives, constrints, and applicability, such a comparison is out of the scope of this thesis. Interested readers please refer to [97] for 
path covering in graph theory.

Table 4.4 Comparison of the cost reduction for HDFS and SPEM

\begin{tabular}{|c|c|c|c|c|c|c|c|c|c|}
\hline Network & Algorithm & $N$ & $\boldsymbol{L}$ & $\begin{array}{c}\text { avg. node } \\
\text { degree }\end{array}$ & $M$ & $G_{n}(\%)$ & $M^{\prime}$ & $M+M^{\prime}$ & $G_{n}^{\prime}(\%)$ \\
\hline \multirow{2}{*}{ NSFNET } & HDFS & \multirow{2}{*}{14} & \multirow{2}{*}{21} & \multirow{2}{*}{3.00} & 6 & 71.4 & 7 & 13 & 38.1 \\
\hline & $\overline{\text { SPEM }}$ & & & & 4 & 80.9 & 15 & 19 & 9.5 \\
\hline \multirow{2}{*}{ ARPA2 } & HDFS & \multirow{2}{*}{21} & \multirow{2}{*}{25} & \multirow{2}{*}{2.38} & 4 & 84.0 & 16 & 20 & 20.0 \\
\hline & SPEM & & & & 4 & 84.0 & 18 & 22 & 12.0 \\
\hline \multirow{2}{*}{ SmallNet } & HDFS & \multirow{2}{*}{10} & \multirow{2}{*}{22} & \multirow{2}{*}{4.40} & 8 & 63.6 & 7 & 15 & 31.8 \\
\hline & SPEM & & & & 4 & 81.8 & 16 & 20 & 9.1 \\
\hline \multirow{2}{*}{ Bellcore } & HDFS & \multirow{2}{*}{15} & \multirow{2}{*}{28} & \multirow{2}{*}{3.73} & 6 & 78.6 & 15 & 21 & 25.0 \\
\hline & SPEM & & & & 5 & 82.1 & 21 & 26 & 7.1 \\
\hline
\end{tabular}

Note: $N$ - The number of nodes in the given network

$L-$ The number of links in the given network;

$M-$ The number of m-cycles

$M^{\prime}-$ The number of extra monitor for achieving complete fault localization

\subsubsection{Time-complexity}

Although the MFDL is an off-line algorithm so the time complexity is not critical, the time complexities of HDFS and SPEM algorithms can be estimated. For HDFS, the DFS itself for each edge has $O(V+L)$ of time complexity [31]. Each cycle consists of at least 3 edges, so the total complexity (worst case) of HDFS algorithm is $O((V+L) L / 3) \sim O\left(L V+L^{2}\right)$, where $V$ is the number of nodes and $L$ is the number of edges. For SPEM, a Euler path can be found in $O(L)$-time. The augment includes finding odd-degree nodes ( $O(V)$-time), finding shortest paths ( $O\left(V^{2}\right)$-time), and comparing 
those paths $(O(V)$-time). The final step for SPEM algorithm is the decomposition of Euler path into cycles, which can be done in $O(V)$-time. So the total complexity of SPEM is $O\left(V^{2}+L\right)$.

-84 - 


\section{Chapter 5}

\section{Condition for Achieving Complete Fault}

\section{Localization}

$\mathrm{T}$

THE previous chapters showed the feasibility of $m$-cycles based FDL approaches and investigated the performances of two $m$-cycle construction algorithms together with a fault localization algorithm, MFLA, in terms of a group of newly defined evaluation metrics such as localization degree, wavelength overhead, as well as cost analyses. In the results of fault localization, as shown in Tables A.1 - 8, in some cases every single network fault can be located to a specific network link, i.e. the complete fault localization is achieved, while in some other cases the complete localization cannot be available. This chapter firstly investigates the necessary and sufficient condition for achieving complete fault localization in AONs, then proposes a spanning-tree based $m$ cycle construction algorithms (called HST algorithm). It is then proven that the HST algorithm produces a set of $m$-cycles that lead to a complete fault localization, if such an $m$-cycle set exists in a given network topology. The HST algorithm is also applied to four typical network examples given in Chapter 4.5 to evaluate its performance in terms of the metrics newly defined in Chapter 3.5.

$-85-$ 


\subsection{Conditions for Complete Fault Localization}

As described before, an AON can be modeled as a finite undirected graph $G(V, E)$, where $V$ and $E$ are sets of nodes and links respectively (see Chapter 3.2). Any network fault can be detected and located by the $m$-cycle based FDL approach. In such FDL approaches, the faulty candidate links are the common part of those $m$-cycles in which alarms are received. Clearly, if each link is covered by a unique combination of $m$-cycles, any single link failure can be located to a specific link, in which case the complete fault localization is achieved. For any two links in a graph with a cycle cover, if there exists at least one cycle that covers one link but not the other one, it is said that these two links are separable. If all links in a graph are separable in pair-wise, a complete fault localization based on $m$-cycles is achievable.

For graph $G(V, E)$, a path of $G$ is called degree-2 chain or simply chain if all of its internal nodes are, but neither of the endpoints is of degree-2. Clearly, if a link in the chain is covered by an $m$-cycle, all other links in the chain will be covered by the same $m$-cycle, i.e., links of a chain are not separable, e.g. links $(2,3)$ and $(3,4)$ in Figure 5.1a. Furthermore, there are cases that two links are connected by a subgraph that all nodes within the subgraph has no connection to any node outside the subgraph except for the two links that are under check. Such a subgraph can be shrunk to a virtual node with degree-2, e.g. links $\left(n_{1}, n_{2}\right)$ and $\left(n_{3}, n_{4}\right)$ in Figure 5.1b. A path is defined as an extended degree-2 chain or simply extended chain, if it consists of such links, sub-graphs, and chains. Following the above observations, a theorem can be claimed as the necessary and sufficient condition for the complete fault localization, 


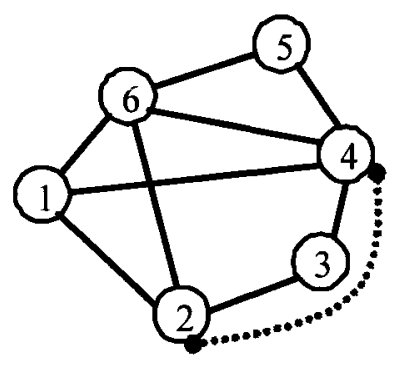

(a)

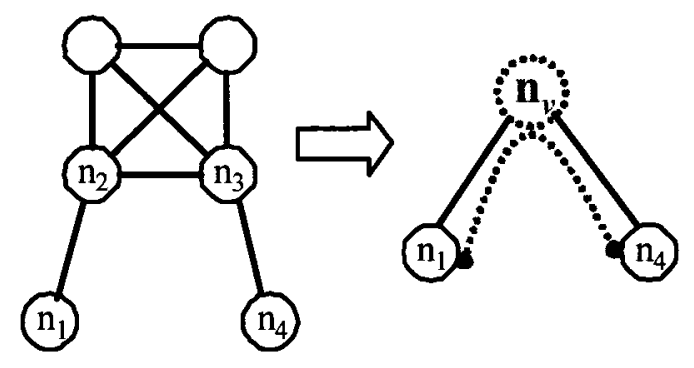

(b)

degree-2 or extended degree-2 chains

Figure 5.1 Example of degree-2 and extended degree-2 chains

With the definition of degree- 2 chain, the following lemma can be claimed,

Lemma 5.1.1: In a graph without degree- 2 chains, given edges $\left(n_{1}, n_{2}\right)$ and $\left(n_{3}, n_{4}\right)$, as shown in Fig 5.2, we can always find a node $n_{c}$ within a path from $n_{2}$ to $n_{3}$ with a new path from $n_{c}$ to either $n_{1}$ or $n_{4}$, which does not travel through $\left(n_{1}, n_{2}\right)$ and $\left(n_{3}, n_{4}\right)$.

Proof: Edges $\left(n_{1}, n_{2}\right)$ and $\left(n_{3}, n_{4}\right)$ in a graph without degree-2 chain are shown in Figure 5.2. If all paths from a node within paths from $n_{2}$ to $n_{3}$ travel through either $\left(n_{1}, n_{2}\right)$, or $\left(n_{3}, n_{4}\right)$, or both, then we remove $\left(n_{1}, n_{2}\right)$ and $\left(n_{3}, n_{4}\right)$ from the graph and the graph becomes two components. Shrink the component containing $n_{2}$ and $n_{3}$ into a virtual node then we find $\left(n_{1}, n_{2}\right)$ and $\left(n_{3}, n_{4}\right)$ are in a virtual degree- 2 chain. This contradicts to the given condition.

Based on Lemma 5.1.1 and the definitions of degree-2 and extended degree- 2 chain, the following theorem is claimed: 


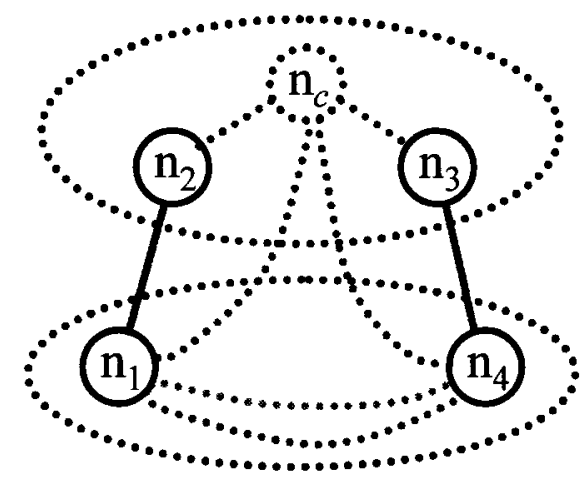

Figure 5.2. Path existence in graph without degree-2 chain

Theorem 5.1.1: A complete fault localization based on $m$-cycles in a connected, bridgeless AON is achievable if and only if the topology of the AON contains no chain and extended chain.

\section{Proof: (A) Necessary}

Assume the complete fault localization is achievable for graph $G$, i.e. any two links are separable. Let $\left(n_{1}, n_{2}\right),\left(n_{3}, n_{4}\right) \in G$ be any two such links. There exist the following cases: (i) Assume cycles covering $\left(n_{1}, n_{2}\right)$ and $\left(n_{3}, n_{4}\right)$ have no common part, like Figure 5.3(a). According to Lemma 5.1.1, there exists at least one path between the endpoints of the two links, e.g. path $P=\left(n_{2} \cdots n_{3}\right)$. Thus $n_{2}$ and $n_{3}$ are of degree- 3 or above and not internal nodes of a chain or extended chain. Similarly, if $n_{1}$ and $n_{4}$ are internal nodes of a chain or extended chain, there must exist a path $P^{\prime}$ connecting them and $\left(n_{1}, n_{2}\right),\left(n_{3}, n_{4}\right) \notin P^{\prime}$, which makes degrees of $n_{1}$ and $n_{4}$ (or another node in $P^{\prime}$ ) larger than two. But this is conflict to the definition of internal nodes. Therefore, $\left(n_{1}, n_{2}\right)$ and $\left(n_{3}, n_{4}\right)$ cannot be in a chain or extended chain. (ii) Assume cycles covering $\left(n_{1}, n_{2}\right)$ and 
$\left(n_{3}, n_{4}\right)$ have a common part that does not contain them, as in Figure 5.3(b), then at least one endpoint of the common part, $n_{c}$, falls into a path between $n_{2}$ and $n_{3}$. With the fact that $n_{c}$ is of degree-3 or above and that $n_{1}, n_{4}$ are not internal nodes of a chain as showed in (i), the two links cannot be in a chain or extended chain. (iii) Assume the two cycles have a common part that contains one of the two links, as in Figure 5.3(c). Similarly to (ii), the two links are not in a chain or extended chain.

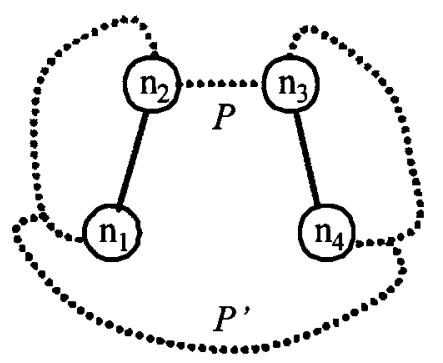

(a)

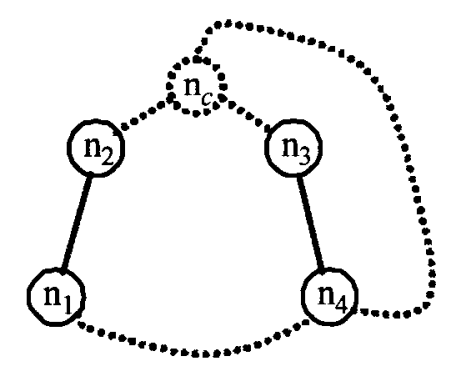

(c)

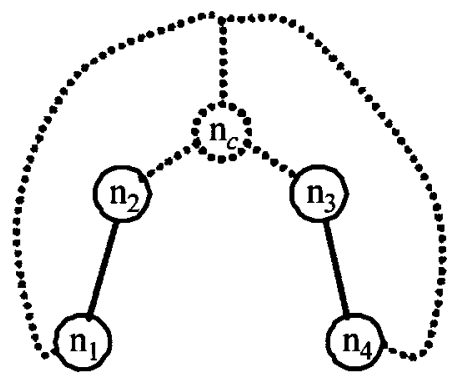

(b)

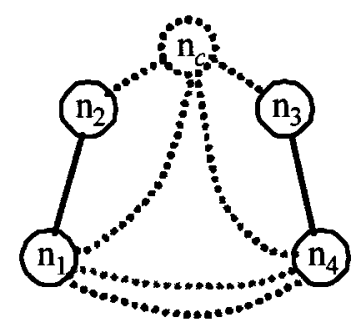

(d)

Figure 5.3 Proof of the necessary and sufficient conditions for complete fault localization

(B) Sufficient

Assume $G$ contains neither chain nor extended chain, but contains two links $\left(n_{1}, n_{2}\right)$ and $\left(n_{3}, n_{4}\right)$ that are not separable, as in Figure 5.3(d). Thus, there exists a node 
or virtual node $n_{c}$ between node pair $\left(n_{2}, n_{3}\right)$, whose degree is 3 or above. To avoid a (extended) chain, there must exist at least one path from $n_{c}$ to either $n_{1}$ or $n_{4}$, or both, which forms "cross straddles". But with the straddle it can immediately constructs two new cycles that cover the two links respectively and thus they are separable now. This is contrary to the assumption.

The following corollary is straightforward from the theorem,

Corollary: All nodes of a network must be of degree-3 or above to achieve the complete fault localization based on $m$-cycles.

The above theorem and corollary are important conclusions. First of all, they have shown that, under some regular conditions, the complete fault localization is achievable. This allows the $m$-cycles based approach to achieve the same performance as monitorper-link approach and, at the same time, reduce the number of required network monitors and therefore the cost of FDL significantly. It is well known that the cost of optical monitors (e.g., OSA, optical tranceiver, etc.) is much higher than that of optical wavelengths due to the DWDM technology, especially for network carriers who already have abundant spare wavelengths. For example, the utilization of wavelengths in metro optical networks is usually lower than $50 \%$ or even close to $10 \%$. In such cases the cost of wavelengths is negligible because the spare wavelengths are already there. Secondly, the theorem and corollary have led us to find and prove that spanning-tree based $m$-cycle construction algorithms (will be described in the following sub-chapters) can create a set of $m$-cycles in which complete fault localization can be achieved, if the AON topology satisfies the condition in Theorem 5.1.1. 


\subsection{Heuristic Spanning-Tree (HST) based Cycle}

\section{Construction Algorithm}

For a connected, bridgeless, simple graph $G(V, E)$, there must exist a spanning-tree $T$. $\forall$ link $e \notin T$ ( $e$ is called as a "chord"), if the two endpoints of $e$ are $n_{1}$ and $n_{2}$, then $n_{1}, n_{2} \in T$ and there must exist a path $p \in T$ that connects $n_{1}$ and $n_{2}$. Thus link $e$ and path $p$ form a cycle. It is said that this cycle is generated by the chord $e$. Each chord generates such a unique cycle. The following cycle cover existence lemma is claimed,

Lemma: There exists at least one cycle cover for a connected, bridgeless, simple graph $G(V, E)$.

Proof: Assume there is a bridgeless graph $G(V, E)$ that has no cycle cover. Then there exists at least one edge $e\left(n_{1}, n_{2}\right) \in E$, which cannot be covered by any cycle. Except for the edge $e$ there is no other path connecting $n_{1}, n_{2}$ (otherwise link $e$ and the path connecting $n_{1}, n_{2}$ form a cycle and a cycle cover can be obtained). Thus if $e$ is removed, $G$ will be disconnected. This is in contradiction to that $G$ is bridgeless.

For a cycle $c_{i}$ in a graph $G$ with precisely $L$ edges, a cycle associative vector $\mathbf{w}_{\mathbf{i}}$

with $L$ components can be assigned to it. The $j$-th component $w_{i}^{j}$ of the vector $\mathbf{w}_{\mathbf{i}}=\left(w_{i}^{1}, w_{i}^{2}, \cdots, w_{i}^{L}\right)$ is one if the $j$-th edge of $G$ lies in $c_{i}$, and zero otherwise (please note the difference with associative code defined in Chapter 3.3). Cycles $c_{1}, c_{2}, \cdots, c_{M}$ 
are called independent if their associative vectors are linearly independent*. H. Walther has proved the following theorem in [75],

Walther's Theorem: Let graph $G(V, E)$ be a connected graph with $L$ edges and $N$ vertices. Then there exist $L-N+1$, but no more independent elementary cycles $†$.

Based on the above lemma and theorem, the following theorem is claimed,

Theorem 5.2.1: For a connected, bridgeless, simple graph $G(V, E)$ with a given spanning-tree, cycles generated by corresponding chords constitute a cycle cover for $G$.

Proof: Let $G(V, E)$ has $L$ edges, $N$ vertices, and the spanning-tree $T$. Then all edges in $E$ can be partitioned into two sets: $N-1$ edges in $T$ and $L-N+1$ edges not in $T$.

(1) Each edge not in $T$ is a chord. It generates a cycle and is covered by this cycle. There are $L-N+1$ such cycles.

(2) Assume there exists a link $e^{*}, e^{*} \in T$, but $e^{*}$ is not covered by any cycle generated by those chords. Because of the lemma, there must exist another cycle $c_{0}$ in which $e^{*}$ appears. The associative vector of $c_{0}$ is $\mathbf{w}_{0}=\left(w_{0}^{1}, w_{0}^{2}, \cdots, w_{0}^{*}, \cdots, w_{0}^{L}\right)$, where $w_{0}^{*}=1$ for the position of edge $e^{*}$. For all other cycles, the components at this position in their associative vectors are zero, because they do not cover edge $e^{*}$. Thus cycle $c_{0}$ is independent from all other $L-N+1$ cycles. Consequently, by adding $c_{0}$ to the $L-N+1$ chord-generated cycles, graph $\mathrm{G}$ has $L-N+2$ independent cycles. This is a contradiction to Walther's theorem.

\footnotetext{
*A group of vectors $\mathbf{w}_{1}, \mathbf{w}_{2}, \cdots, \mathbf{w}_{\mathbf{n}}$ are linearly independent if $\sum_{i=1}^{i=n_{i}} k_{i} \mathbf{w}_{\mathbf{i}}=0$ holds only when $k_{1}=k_{2}=\cdots=k_{n}=0$.

${ }^{\dagger}$ A cycle is called elementary cycle if no vertex is encountered more than once when traversing it. Each cycle can be partitioned into elementary cycles. In this chapter, a cycle is an elementary cycle.
} 
In the proof, please note that a cycle cover of graph $\mathrm{G}$ is uniquely determined by the given spanning-tree.

Among the numerous existing cycle building algorithms, the spanning-tree based ones are fast, simple and flexible. Breadth-first and depth-first spanning-trees (BFST and DFST) are well known and have been in common use for a long time. Numerous algorithms to generate such spanning-trees have been intensively studied [73]. Figure 5.4 gives three spanning-trees for an example graph and $m$-cycles generated by corresponding chords. Numbers of cycles in the covers generated by various spanningtrees are the same for the graph. By enumerating the faulty candidates for all possible alarm codes, it is found that localization degrees for them are also the same $(I=1)$. However, the figure shows that the average cover depth (i.e. the average wavelength overhead) per link is smaller for the cover generated by BFST than by DFST. Furthermore, the average cover depth might be decreased by including nodes with large degrees in the tree (comparing Figures $5.4 \mathrm{~b}$ and $5.4 \mathrm{c}$ ). This observation leads us to choose BFST and apply a heuristic rule of putting the large-degree nodes into the spanning-tree as early as possible while generating the spanning-tree for constructing a cycle cover. A heuristic spanning-tree (HST) based cycle construction algorithm [76] is then given below,

1) Initial: for a graph $G(V, E)$, label the degrees of all nodes; set the spanning-tree $T=n u l l ;$ select the node with the maximum degree as the root. Add all links to $T$ that are incident to the root;

2) For each node $n_{i} \in T$, update its degree label with the number of links that are incident to $n_{i}$ and connect $n_{i}$ with nodes not in $T$; 
3) Select the node with the maximum degree label in $T$, add all links to $T$ that are incident to the selected node and connect it with nodes not in $T$;

4) Repeat step 2)-3) until all node-degree labels are zero. Now $T$ is a spanning-tree of $G(V, E)$;

5) Given $T$, construct the cycles for all chords, they form a cycle cover and are the required $m$-cycles;

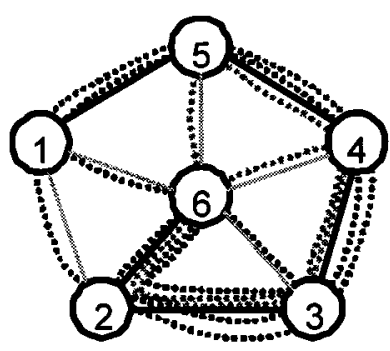

(a)

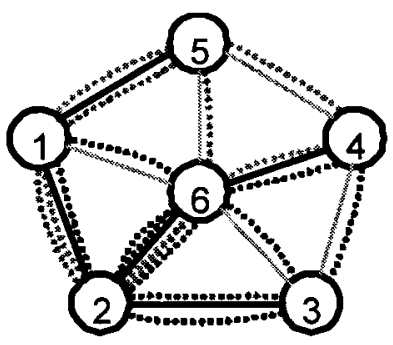

(b)

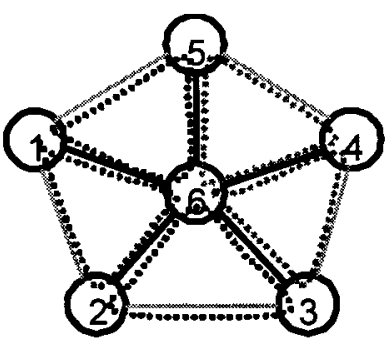

(c)

Figure 5.4 Spanning-tree and cycle cover (links in spanning-tree are in bold)

(a) DFST: max cover depth $=5 /$ link, average cover depth $=2.3 /$ link

(b) BFST (rooted from random nodes): max cover depth $=5 /$ link, average cover depth $=1.9 /$ link

(c) BFST (rooted from the node with maximum degree): $\max$ cover depth $=2 /$ link, average cover depth $=1.5 /$ link

Figure 5.5 demonstrates the above HST algorithm by applying it to the network example given in Figure 3.1. Firstly, all node degrees are labeled beside the nodes and node 6 has the maximum degree, 6 . Thus node 6 is selected in the first iteration and all links incident to it (in lines) are added to the partial spanning-tree $T, T=\{(6,4),(6,5)$, $(6,7),(6,8),(6,9),(6,10)\}$. Then nodes in the partial spanning-tree update their degree by the number of links that are incident to them and connect with nodes not in $T$. Updated 
degrees after this iteration are shown in the round brackets besides the original degree numbers. At the second iteration, node 4 is selected and new degrees are shown in square brackets. Repeat such process, finally a spanning-tree is generated as in Figure 5.5(b).

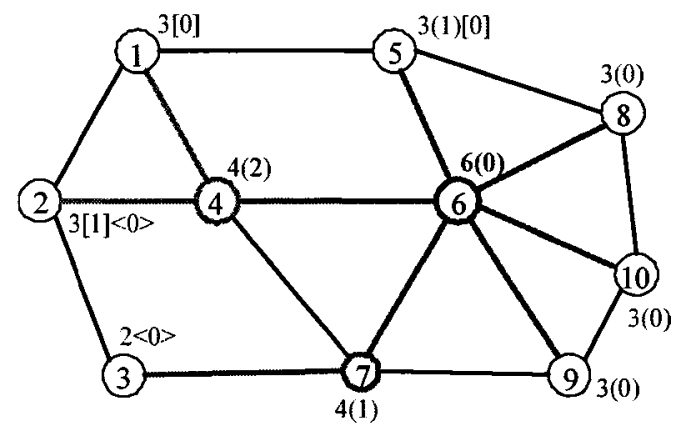

(a)

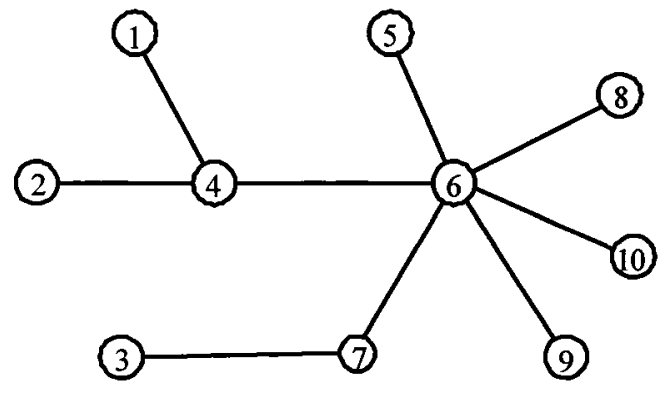

(b)

Figure 5.5 Demonstration of the HST algorithm

(a) Demonstration of the HST algorithm;

(b) The generated spanning-tree

Remark: For fault localization with $m$-cycles obtained by HST algorithm, each chord appears in a unique $m$-cycle and thus it can be completely localized if faults occur upon such edges. Most edges in $T$ are also completely localizable for topologies of real telecommunication networks, although it is not guaranteed all the time. Therefore the lower bound ratio of localizable links is

$$
\text { (localizable link) } \%=\frac{L-N+1}{L}=1+1 / L-2 / \bar{d}
$$

where $\bar{d}=2 N / L$ is the average node degree of graph $G$. This lower bound shows that the ratio of localizable links in a graph is higher when the average node degree is higher. It implies that the HST algorithm has better performance in terms of localization degree for more complex networks. 


\subsection{Complete Fault Localization and Spanning-Tree (ST) Based $M$-Cycle Construction Algorithms}

A spanning-tree (ST) based $m$-cycle construction algorithm has been developed in Chapter 5.2. As described in Chapter 5.2, for graph $G(V, E)$, Theorem 5.2.1 proves that cycles generated by all chords construct a cycle cover for G. Here the following theorem on ST based $m$-cycle construction algorithms can be proven:

Theorem 5.3.1: The complete fault localization is achievable for a given graph with the $m$-cycle set generated by a ST based construction algorithm, if the graph satisfies the condition in theorem 5.1.1.

Proof: $\forall e_{1}, e_{2} \in G$, for graph $G$ with any spanning-tree $T$ and a cycle cover generated by the ST cycle construction algorithm:

(1) If $e_{1}, e_{2} \notin T: e_{1}$ and $e_{2}$ are chords and covered by the different cycles generated by themselves respectively, thus they are separable;

(2) If $e_{1}, e_{2} \in T$ : Without the loss of generality, let links $e_{1}$ and $e_{2}$ be $\left(n_{1}, n_{2}\right)$ and $\left(n_{3}, n_{4}\right)$ respectively, as shown in Figure 5.6a. Because there is no chain or extended chain in $\mathrm{G}$, there must exist a path connecting $e_{1}$ and $e_{2}$, and a node $n_{c}$ in this path whose degree is 3 or above. Note that in Figure 5.6a, $n_{c}$ (might be a shrunk virtual node) could be overlapped with $n_{2}$ or $n_{3}$ or both. Due to the bridgelessness of $G$, there is a path (in the tree) from $n_{c}$ to $n_{1}$ and to $n_{4}$ respectively, which is other than $n_{c}-n_{2}-n_{1}$ 
and $n_{c}-n_{3}-n_{4}$ respectively (see Lemma 5.1.1). Let $P_{c}$ be the common part of these two paths, $P_{a}$ and $P_{b}$ be the different part of them to node $n_{1}$ and $n_{4}$ respectively. At least two of $\left\{P_{a}, P_{b}, P_{c}\right\}$ contain one or more chords; otherwise a cycle would be formed in the tree. Let $P_{a}$ and $P_{b}$ contain one chord respectively. The cycle generated by the chord in $P_{a}$ covers $e_{1}$, while the cycle generated by the chord in $P_{b}$ covers $e_{2}$. Thus $e_{1}$ and $e_{2}$ are separable;

(3) If $e_{1} \in T, e_{2} \notin T$ : As in Figure 5.6b, it is similar to (2) but now a chord can be found in $\left\{P_{a}, P_{c}\right\}$ (no chain or extended chain in the graph). The cycle generated by this chord covers $e_{1}$ but not $e_{2}$. Thus $e_{1}$ and $e_{2}$ are separable.

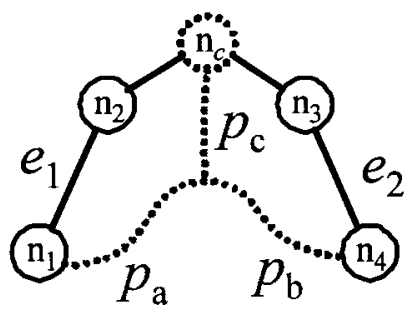

(a)

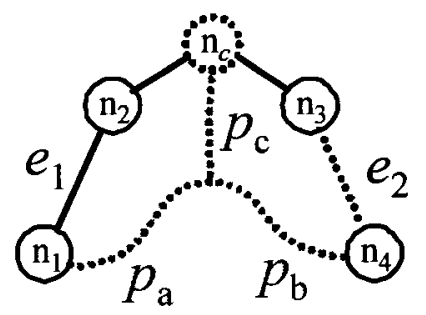

(b)

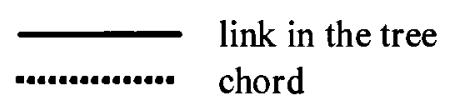

Figure 5.6 Proof of Theorem 5.3.1

With the arbitrary selection of $e_{1}$ and $e_{2}$, any link pair in $G$ is separable. Therefore the complete fault localization can be achieved with cycles generated by the ST construction algorithm. 
Theorem 5.3.1 provides us a way to find an $m$-cycle set in which the complete fault localization can be obtained for a given mesh $\mathrm{AON}$, if the topology of that AON satisfies the condition described in Theorem 5.1.1.

\subsection{Examples and Performance Evaluation}

To evaluate the performances of the $m$-cycle construction algorithm developed in this chapter, the HST algorithm is applied together with the MFLA (see Chapter 3.3) to four typical example networks (NSFNET, ARPA2, SmallNet, and Bellcore) given in Chapter 4.5. Their topologies are shown in Figure 4.4. The $m$-cycles obtained by the HST $m$-cycle construction algorithm in the four network examples are listed in Figure B.1 in appendix B of this thesis. With the obtained $m$-cycles, the MFLA is applied to those network topologies and list all possible alarm codes with corresponding fault localization results from Tables B. 1 to B. 4 in the appendix of this thesis. In this section, the performance of the HST $m$-cycle construction algorithm is evaluated in terms of those metrics described in Chapter 3.5, including the localization degree of MFLA, wavelength overhead due to $m$-cycles, and the cost reduction over monitor-per-link method. The evaluation results are compared with the HDFS and SPEM algorithms developed in Chapter 4.

\subsubsection{Localization degree}

$M$-cycles obtained by HST algorithm in the four network examples are listed in Figure B.1 (see Appendix B). Tables B.1 - B.4 list faulty candidate sets for all possible 
alarm codes in those examples with $m$-cycles obtained by HST algorithm. Then the localization degrees can be immediately calculated according to equation 3.5.1. Similar to the simulation for HDFS and SPEM algorithms, here the localization results are based on the single-failure scenario for simplicity, but they could be easily extended to multiplefailure scenarios by including the alarm combinations in the tables for multiple-failure scenarios, as described in the end of Chapter 3.3.

Table 5.1 summarizes those localization results for HST algorithm and compares the localization degrees in network examples with $m$-cycles respectively obtained by the HDFS and SPEM algorithms reported in Chapter $4.3-4.5$. In the table, the maximum candidate set size is an indication of the worst-case alarm code in fault localization. The comparison shows that the HST algorithm has the best while SPEM algorithm has the worst performance in terms of the fault localization degree. The HDFS algorithm is a tradeoff between them. Further analyses indicate that the HST algorithm performs even better in terms of localization degree for graphs with larger average node degrees. More specifically, for graphs with average node degree larger than 3.0 , the localization degrees for using HST algorithm are very close to the ideal case, complete localization $\left(I_{\text {ideal }}=1\right)$. This observation implies that such FDL approaches are suitable for complex networks (with large average node degree), and are scalable. Additionally, even for the worst case, ARPA2, the localization degree for using HST algorithm is $I=2.5$, which is not far away from the complete localization. Therefore, the proposal for fault detection and localization is technically feasible for mesh AONs. 
Table 5.1 Comparison of MFLA results for $m$-cycle construction algorithms

\begin{tabular}{|c|c|c|c|c|c|c|}
\hline Network & Algorithm & $N$ & $\boldsymbol{L}$ & $\begin{array}{l}\text { avg. node } \\
\text { degree }\end{array}$ & $\begin{array}{c}\text { localization } \\
\text { degree }\end{array}$ & $\begin{array}{c}\text { max candidate } \\
\text { set size }\end{array}$ \\
\hline \multirow{3}{*}{ NSFNET } & HST & \multirow{3}{*}{14} & \multirow{3}{*}{21} & \multirow{3}{*}{3.00} & 1.105 & 2 \\
\hline & HDFS & & & & 1.500 & 3 \\
\hline & SPEM & & & & 3.000 & 7 \\
\hline \multirow{3}{*}{ ARPA2 } & HST & \multirow{3}{*}{21} & \multirow{3}{*}{25} & \multirow{3}{*}{2.38} & 2.500 & 6 \\
\hline & HDFS & & & & 3.130 & 6 \\
\hline & SPEM & & & & 5.000 & 8 \\
\hline \multirow{3}{*}{ SmallNet } & HST & \multirow{3}{*}{10} & \multirow{3}{*}{22} & \multirow{3}{*}{4.40} & 1.000 & 1 \\
\hline & HDFS & & & & 1.470 & 3 \\
\hline & SPEM & & & & 3.670 & 6 \\
\hline \multirow{3}{*}{ Bellcore } & HST & \multirow{3}{*}{15} & \multirow{3}{*}{28} & \multirow{3}{*}{3.73} & 1.077 & 2 \\
\hline & HDFS & & & & 2.150 & 6 \\
\hline & SPEM & & & & 4.670 & 8 \\
\hline
\end{tabular}

\subsubsection{Wavelength overhead}

The cost of wavelength channels is evaluated by the reserved wavelength for $m$ cycles in network links, i.e. wavelength overhead defined in Chapter 3.5. The numbers of maximum and average reserved wavelengths in links using HST algorithm are summarized and compared with HDFS and SPEM algorithms in Table 5.2. It shows that numbers of both maximum and average reserved wavelengths for $m$-cycles obtained by HST algorithm are larger than HDFS and SPEM. This is the payment for the benefit in localization degree for this $m$-cycle construction algorithm. The SPEM algorithm has the minimum number of reserved wavelengths and thus introduces the minimum wavelength 
overhead, while the HDFS algorithm is a trade-off between SPEM and HST algorithms. As described in Chapter 4.5.2, in average case wavlength overheads brought by the HST algorithm have trivial impact on network utilization, if such impact is not negligible. Even for a small number of available wavelengths per link, e.g. $\Delta=64$, the wavelength overheads for SPEM algorithm is less than $2 \%$ for all example networks. For the worst case with HST algorithm, the wavelength overheads are still very small (around $4 \%$, see Table 5.2).

Table 5.2 Comparison of wavelength overhead for $m$-cycle construction algorithms

\begin{tabular}{|c|c|c|c|c|c|c|c|c|}
\hline Network & Algorithm & $N$ & $L$ & $\begin{array}{c}\text { avg. node } \\
\text { degree }\end{array}$ & $\Lambda_{\text {avg }}$ & $\Lambda_{\max }$ & $\begin{array}{c}W O H_{\text {avg }} \\
(\%)\end{array}$ & $\begin{array}{c}W O H_{\max } \\
\text { (\%) }\end{array}$ \\
\hline \multirow{3}{*}{ NSFNET } & HST & \multirow{3}{*}{14} & \multirow{3}{*}{21} & \multirow{3}{*}{3.00} & 1.90 & 5 & 2.97 & 7.81 \\
\hline & HDFS & & & & 1.57 & 3 & 2.45 & 4.69 \\
\hline & $\overline{\text { SPEM }}$ & & & & 1.24 & 2 & 1.93 & 3.12 \\
\hline \multirow{3}{*}{ ARPA2 } & HST & \multirow{3}{*}{21} & \multirow{3}{*}{25} & \multirow{3}{*}{2.38} & 1.60 & 3 & 2.50 & 4.69 \\
\hline & HDFS & & & & 1.36 & 3 & 2.12 & 4.69 \\
\hline & SPEM & & & & 1.20 & 2 & 1.87 & 3.12 \\
\hline \multirow{3}{*}{ SmallNet } & HST & \multirow{3}{*}{10} & \multirow{3}{*}{22} & \multirow{3}{*}{4.40} & 1.95 & 6 & 3.05 & 9.37 \\
\hline & HDFS & & & & 1.55 & 3 & 2.42 & 4.69 \\
\hline & SPEM & & & & 1.18 & 2 & 1.84 & 3.12 \\
\hline \multirow{3}{*}{ Bellcore } & HST & \multirow{3}{*}{15} & \multirow{3}{*}{28} & \multirow{3}{*}{3.73} & 1.96 & 8 & 3.06 & 12.5 \\
\hline & HDFS & & & & 1.43 & 3 & 2.23 & 4.69 \\
\hline & SPEM & & & & 1.14 & 2 & 1.78 & 3.12 \\
\hline
\end{tabular}

Note: $\mathrm{WOH}_{\text {avg }}$ and $\mathrm{WOH}_{\max }$ are in percentage and calculated for $\Delta=64$. 


\subsubsection{Cost reduction}

The cost of the proposed FDL scheme is measured by both the number of required monitors and reserved wavelengths for $m$-cycles. The cost of monitors is weighted by the number of $m$-cycles (denoted as $M$ ), which is equal to the number of required monitors. A cost reduction $\left(G_{n}\right)$ can be calculated as equation 3.5 .8 in comparison with the brute force monitor-per-link method. To evaluate the costs for the proposed $m$-cycle based FDL approach, the values of $M$ and the cost reduction is listed in Table 5.3 for all example networks with $m$-cycles obtained by HDFS, SPEM and HST algorithms, respectively. Clearly, the SPEM algorithm produces the minimum number of $m$-cycles and thus has the maximum the cost reduction in the example networks, while the HST algorithm has the minimum. Specifically, SPEM algorithm saves more than $80 \%$ of monitors in the monitor-per-link method. For the worst case with HST algorithm, although the $M$ values are larger than HDFS and SPEM, it still has good cost reduction. The saving for HST algorithm are in the range of $40-60 \%$ for example networks.

As discussed in Chapter 3.5, for a fair comparison with the monitor-per-link method, $M^{\prime}$ extra monitors are added to those links that cannot be fully localized under the MFLA to achieve complete localization. Then the cost reduction calculation is revised as equation 3.5.9. The revised cost reduction obtained from the four example networks with $m$-cycles obtained by HDFS, SPEM and HST algorithms is also calculated respectively in Table 5.3. Certainly the cost reduction decreases due to the extra monitors. But interestingly, with the supplement monitors, the cost reduction for the HST algorithm goes to the first rank among the proposed $m$-cycle construction algorithms, while the HST algorithm also achieves the best localization degree. It indicates that the HST 
algorithm seems to be the most promising one. Again, the average node degree of a graph affects the cost reduction. For graphs whose average node degree is 3.0 or above, the cost reduction is $40-52 \%$. Even for the worst case, ARPA2 (average node degree 2.38), HST algorithm still has $20 \%$ cost reduction over the brute force case. Additionally, the comparison with monitor-per-link schemes analyzed in Chapter 4.5.3 is also applicable here and the significant cost reduction over monitor-per-path schemes can be observed.

Table 5.3 Comparison of the cost reduction for $m$-cycle construction algorithms

\begin{tabular}{|c|c|c|c|c|c|c|c|c|c|}
\hline Network & Algorithm & $N$ & $L$ & $\begin{array}{c}\text { avg. node } \\
\text { degree }\end{array}$ & $\boldsymbol{M}$ & $G_{n}(\%)$ & $M^{\prime}$ & $M+M^{\prime}$ & $G_{n}^{\prime}(\%)$ \\
\hline \multirow{3}{*}{ NSFNET } & HST & \multirow{3}{*}{14} & \multirow{3}{*}{21} & \multirow{3}{*}{3.00} & 8 & 61.9 & 2 & 10 & 52.4 \\
\hline & HDFS & & & & 6 & 71.4 & 7 & 13 & 38.1 \\
\hline & SPEM & & & & 4 & 80.9 & 15 & 19 & 9.5 \\
\hline \multirow{3}{*}{ ARPA2 } & HST & \multirow{3}{*}{21} & \multirow{3}{*}{25} & \multirow{3}{*}{2.38} & 5 & 80.0 & 15 & 20 & 20.0 \\
\hline & HDFS & & & & 4 & 84.0 & 16 & 20 & 20.0 \\
\hline & SPEM & & & & 4 & 84.0 & 18 & 22 & 12.0 \\
\hline \multirow{3}{*}{ SmallNet } & HST & \multirow{3}{*}{10} & \multirow{3}{*}{22} & \multirow{3}{*}{4.40} & 13 & 40.9 & 0 & 13 & 40.9 \\
\hline & HDFS & & & & 8 & 63.6 & 7 & 15 & 31.8 \\
\hline & SPEM & & & & 4 & 81.8 & 16 & 20 & 9.1 \\
\hline \multirow{3}{*}{ Bellcore } & HST & \multirow{3}{*}{15} & \multirow{3}{*}{28} & \multirow{3}{*}{3.73} & 14 & 50.0 & 2 & 16 & 42.9 \\
\hline & HDFS & & & & 6 & 78.6 & 15 & 21 & 25.0 \\
\hline & SPEM & & & & 5 & 82.1 & 21 & 26 & 7.1 \\
\hline
\end{tabular}

Note: $N$-The number of nodes in the given network

$L-$ The number of links in the given network;

$M-$ The number of $m$-cycles

$M^{\prime}-$ The number of extra monitor for achieving complete fault localization 


\subsubsection{Complexity of HST algorithm}

For HST algorithm, the complexity of producing the spanning-tree $T$ is $O\left(V^{2}\right)$ [32]. For a chord, e.g., $\left(n_{1}, n_{2}\right)$, a cycle can be established by finding the path $\left(n_{1}-\cdots-n_{2}\right) \in T$ and adding the chord itself. Each chord generates a cycle. The path within the spanning-tree can be found in $O(V+L)$-time complexity, e.g., using Dijkstra algorithm [31]. Thus, the total complexity of the HST algorithm is $O\left(V^{2}+L(V+L)\right) \sim O\left(V^{2}+V L+L^{2}\right)$. The complexity comparison for HDFS, SPEM and HST is sumarrized in Table 5.4.

Table 5.4. Comparison of time complexity for HDFS, SPEM and HST algorithms

\begin{tabular}{c|c|c|c}
\hline Algorithm & HDFS & SPEM & HST \\
\hline Complexity & $O\left(L V+L^{2}\right)$ & $O\left(V^{2}+L\right)$ & $O\left(V^{2}+V L+L^{2}\right)$ \\
\hline
\end{tabular}




\section{Chapter 6}

\section{Full Formulation of $M$-Cycle}

\section{Construction}

$\mathrm{I}$

$\mathrm{N}$ Chapter $4.3-4.5$, it is shown that the performance of the proposed $m$-cycles based FDL approaches highly depends on how to choose $m$-cycles for a given network topology. Once the $m$-cycle cover is established, the fault localization is fast with low complexity and cost. The three proposed $m$-cycle construction algorithms: HDFS, SPEM, and HST algorithms, have advantages and disadvantages respectively in terms of the evaluation metrics such as fault localization degree, wavelength overhead, as well as cost $[72,74,76,85]$. Furthermore, it is proven in Chapter 5 that the HST algorithm could produce an $m$-cycle cover to implement the complete fault localization, if such $m$-cycle covers exist. However, those $m$-cycle construction algorithms cannot guarantee to produce the minimum number of necessary $m$-cycles, which is equal to the number of required network monitors. Also, the wavelength overhead is not guaranteed to be the minimum. One more consideration is the limit of cycle length rising from the fact in the real world that a lightpath exceeding a certain length, e.g., 16 hops, will involve extra optical amplification and/or re-generation. Therefore, the formal expression of this problem and difficulties for solving it are explored in this chapter. The method is to 
formulate the $m$-cycle construction as a variant constrained cycle cover problem (vCCCP) that takes the above factors into accounts, and explore a branch-and-bound (B\&B) based method to solve the problem with reasonable complexity. The target algorithm should be flexible to include both the wavelength overhead (as the measurement of the reserved wavelength for monitoring) and cycle number (as the measurement of the monitor number) into the cost function for branch-and-bound.

\subsection{Formal definition of variant constrained cycle cover problem (vCCCP)}

The definition of CCP and existing algorithms for solving CCP has been introduced in Chapters 4.1 and 4.2. Recently, a constraint of cycle length has been put on the CCP. This constraint arises in the design of self-healing ring for optical networks. The lightpath exceeding a certain length requires extra optical amplifiers and management, and thus should be avoided in the design. The CCP with the constraint of cycle length is defined as the constrained cycle cover problem (CCCP). It was shown that CCCP is NP-hard [98]. Therefore, some heuristic algorithms have been proposed for CCCP. Reference [98] described a two-step process for finding acceptable solutions to CCCP. Firstly, the algorithm generates a set of candidate cycles, which are obtained by creating one shortest cycle with allowed length for each edge $\left(v_{i}, v_{j}\right)$ and node $v$. Then the set-covering algorithm is applied to extract a minimum-cost subset of the candidate cycles that cover 
all edges in the graph. The set-covering problem itself is also NP-hard [99] and solved by either integer linear programming or branch-and-bound in exponential time complexity.

The algorithm proposed in [100] for CCCP is based on Eulerian matching. It firstly makes the given graph Eulerian through a minimum-cost augmentation. The algorithm only continues when the minimum-cost augmentation yields feasible solutions. Then the same heuristics as in [96] are applied to optimize the CCCP solution.

The greedy algorithm in [97] is also applicable to CCCP if the cycle length limit is considered in the step of choosing the most cost-effective feasible cycle.

A variant version of CCCP can be defined as the CCCP that further bounds the CCCP with the constraint of complete fault localization (denote as vCCCP). The necessary and sufficient conditions for the existence of solutions for this problem are discussed in Chapter 5. Here it is assumed that the given graph satisfies such conditions and that there exist feasible solutions for the vCCCP. Some special transforms (will be discussed in details in Chapter 6.4) may be manipulated to make a network topology satisfy the conditions for complete fault localization, if it does not satisfy such conditions.

The vCCCP is formally defined here as the cycle cover problem with the constraints of both lightpath length limit and complete fault localization. The formal problem definition is described in Figure 6.1. In the description, the first term of the objective function, $\alpha M$, represents the cost of monitoring devices, where $M$ is the number of $m$ cycles in the cover solution. The second term, $\sum_{e \in E} \beta_{e} x_{e}$, is the cost of all wavelengths reserved for monitoring. $E_{e}$ is the set of extra copies of link $e$ for building the $m$-cycle cover. A $k$-cycle is a cycle of size at most $k$, and the $k$-cycle cover is defined as a cycle cover in which each cycle has a length no more than $k[61,98]$. 
Problem Name: variant Constrained Cycle-Cover Problem (vCCCP)

Instance: Given a graph $G(V, E)$ that satisfies the conditions for complete fault localization, a cost coefficient, $\beta_{i}$, associated with one reserved wavelength in link $e_{i}$, a constant cost $\alpha$ designated to each m-cycle, an $x_{i}$ as the number of cycles that cover link $e_{i}$, an associative code $\mathbf{a}_{i}$ for link $e_{i}$, and an integer $k$ as cycle length limit in hops.

Optimization Problem: Minimize $\alpha M+\sum_{e_{i} \in E} \beta_{i} x_{i}$ such that $E \cup \bigcup_{e_{i} \in E} E_{e_{i}}$ can be partitioned into a $k$-cycle cover of $G(V, E)$ and the associative code $\mathbf{a}_{i}$ for each link is unique (i.e., no duplication in the set of associative codes for all links), or show that the problem is infeasible.

Figure 6.1 Formal definition of vCCCP

\subsection{Solving vCCCP}

\subsubsection{Two-phase B\&B algorithm}

To solve the vCCCP problem, a two-phase branch-and-bound (B\&B) based algorithm is developed. The first phase is the shortest-path candidate-cycle construction and the second phase is the B\&B searching for optimal solution among the candidate cycles built in the first phase. The shortest-path candidate-cycle construction (SPCC) is to generate a set of candidate cycles by creating the shortest cycle for every combination of each edge $\left(v_{i}, v_{j}\right)$ and any node $v_{0}$ in graph $G(V, E)$. For each edge $\left(v_{i}, v_{j}\right)$, by temporarily removing the edge from the graph and applying the Dijkstra algorithm (or any other shortest path algorithms, e.g., Bellman-Ford algorithm [36]), it is not difficult to find the 
shortest paths $P_{i v}$ and $P_{v j}$, from node $v_{0}$ to two end nodes of edge $\left(v_{i}, v_{j}\right)$ respectively (please note the ties are included). Paths $P_{i v}$ and $P_{v j}$, together with edge $\left(v_{i}, v_{j}\right)$, constitute a cycle. This cycle is the shortest cycle that covers the given edge $\left(v_{i}, v_{j}\right)$ and node $v_{0}$. Repeating this procedure to find the shortest cycles for all edge-node combinations in $G(V, E)$. Such shortest cycles are candidate ones for the final solution.

Once candidate cycles are established, the algorithm calculates the length of each candidate cycle and sorts them according to their lengths, then deletes the repeated cycles and bad cycles. The so-called bad cycles include cycles that contain only two or less links, or that visit a node more than one time, or with cycle lengths more than the limit $k$. Furthermore, the algorithm checks the existence of feasible solutions based on these cycles and if necessary, builds some extra candidate cycles to guarantee the existence of feasible solutions (details in Chapter 6.2.2).

Then the algorithm enters into the second phase. In this phase, the algorithm checks cycles one by one from that with the least cycle length to that with the largest length in the ordered candidate cycle set. The B\&B algorithm is applied to search feasible solutions and find the near-optimal solution(s) by comparing those feasible solutions (details in Chapter 6.2.3). The B\&B based cycle selection minimizes the cover length and cycle number if the cost function in the vCCCP definition is applied for the bound check. The description of the two-phase algorithm is shown in Figure 6.2

The method to check the link coverage and complete fault localization will be discussed in the remainder of this sub-section, followed by the details of the B\&B algorithm. A descriptive example will be given in Chapter 6.3 to explain the vCCCP solving procedure. 
Algorithm: variant Constrained Cycle-Cover Problem Algorithm

Input: A graph $G(V, E)$ and an integer $k$

Output: A set of $m$-cycles that cover all links in the graph and achieve complete fault localization

Phase 1: Generating candidate cycles

$\xi \leftarrow \phi$

For all $(i, j) \in E$

For all $v \in V \backslash\{i, j\}\{$

Find a minimum-cost path $P_{i v}$ between $(i, v)$ by Dijkstra algorithm;

Find a minimum-cost path $P_{v j}$ between $(v, j)$ by Dijkstra algorithm; If $\exists P_{i v}, P_{v j}$ Then $C \leftarrow P_{i v} \cup(i, j) \cup P_{v j}$;

If $|C| \leq k$ Then $\xi \leftarrow \xi \cup C$;

\}

Phase 2: Select the minimum-cost $m$-cycle cover from the candidate cycle set $\omega \leftarrow 0$

For all $C_{i} \in \xi$

For all $e \in C_{i}$

$\omega_{i} \leftarrow \omega_{i}+\beta_{e} ;$

Sort $\xi$ by $\omega$;

Check all $C_{i} \in \xi$, delete bad cycles and repeated cycles;

Check existence of feasible solution and build extra cycles if necessary

Call Branch-and-Bound algorithm to generate the minimum-cost m-cycle cover.

Figure 6.2 The vCCCP algorithm

\subsubsection{Link coverage, complete fault localization, and existence of feasible solution}

For a given set of cycles, a checking-matrix is established using the binary associative bits defined in Chapter 3.3, as shown in Figure 6.3. In the checking-matrix, each edge has 
a column vector whose elements are associative bits corresponding to $m$-cycles in the tentative solution. If an all-zero column vector is found, it means that there is no cycle includes this edge in the tentative solution and thus the coverage constraint is not yet satisfied. It needs to add more cycles to the tentative solution to cover the whole graph.

The constraint of complete fault localization is checked through pairwisely comparing all the column vectors in the matrix. If two or more column vectors are exactly the same in the matrix, it means that for the alarm code (defined in Chapter 3.3) equal to this column vector, the fault localization results will include all these edges having the same column vector. Thus the constraint of complete fault localization is violated and more cycles need to be added to the candidate solution.

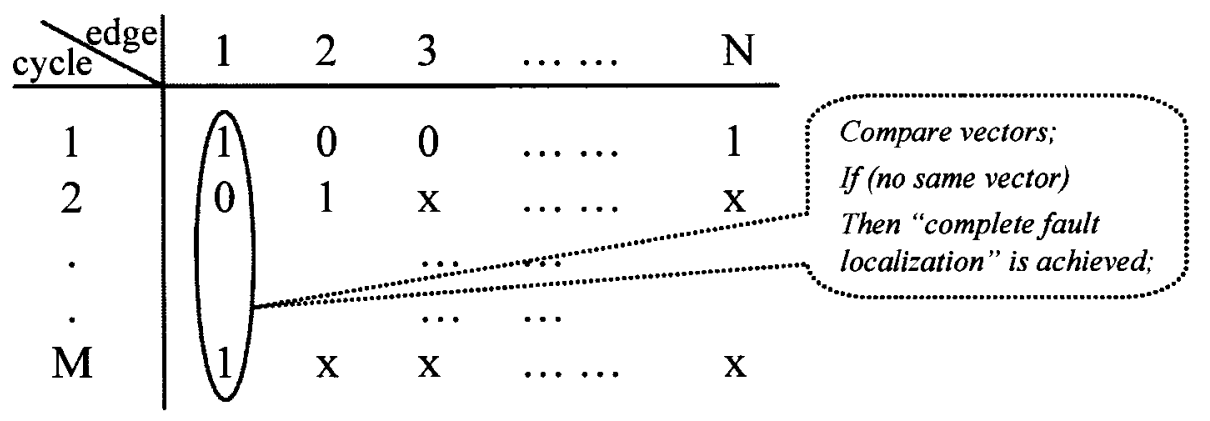

Figure 6.3 Check the coverage and complete fault localization for a cycle set

The above process for checking complete fault localization provides a simple method to guarantee the existence of feasible solutions consisting of candidate cycles built by SPCC. Before entering the second phase of the algorithm, the algorithm constructs the checking-matrix using all candidate cycles produced by SPCC, and compare each column vector with the others. If there exist two or more identical vectors, it cannot achieve 
complete fault localization even add all candidate cycles into the solution, i.e., the feasible solution does not exist. The edges with the identical column vector are not separable. In this case, the algorithm randomly selects one edge from the group of edges with the same vector, temporarily removes this group of edges from the graph, and builds new cycles by applying SPCC for the combination of the selected edge and the nodes of other edges with the identical column vector. For example, if links $e_{1}, e_{2} \in E$ have the same column vector, they are not separable. Denote the end nodes of $e_{1}$ and $e_{2}$ as $\left\{n_{1}, n_{2}\right\}$ and $\left\{n_{3}, n_{4}\right\}$ respectively. Then build new cycles for both edge-node combinations $\left\{e_{1}, n_{3}\right\}$ and $\left\{e_{1}, n_{4}\right\}$ in graph $G\left(V, E \backslash\left\{e_{2}\right\}\right)$ by applying SPCC. Add the new cycles into the candidate cycle set and they will only cover edge $e_{1}$ but not $e_{2}$. In this way, the algorithm makes all edges separable based on the candidate cycles, and thus guarantee the existence of feasible solutions (it is assumed that the topology of the given AON meets the conditions for complete fault localization described in Theorem 5.1.1). Please note that cycles built at this step will not be deleted from the candidate cycle set even their lengths exceed the cycle-length limit, $k$, given at the algorithm initialization. Furthermore, the newly built cycles are inserted into the sorted candidate-cycle set according to their cycle length and the length order in the set is still maintained.

\subsubsection{Branch-and-bound (B\&B) algorithm}

Branch-and-bound algorithms are standard techniques for solving integerprogramming problems by efficiently enumerating the points in a sub-problem's feasible region [102]. The branch-and-bound $(\mathrm{B} \& \mathrm{~B})$ algorithm for the vCCCP problem firstly 
constructs a full binary tree. Nodes at the same level of the tree represent the same candidate cycle, as shown in Figure 6.4. The tree levels are labeled in ascending nonnegative integers from the root to the level of leaves. A candidate cycle is designated to each tree level from the root to the leaf-level in the ascending order of cycle length, i.e., the cycle closer to the root has the smaller length. The tree level label also works as cycle number. For the convenience of implementation in $\mathrm{C}++$ coding, the tree starts from a virtual root node that corresponds to a null cycle with the length of zero. Every route from the root to a leaf in the tree represents a combination of all candidate cycles. Each node along the route is assigned with the value of one if the cycle corresponding to this node is selected in the combination, and assign with the value of zero otherwise. Figure 6.4 is such a full binary tree and shows an example route from the root to a leaf in bold lines. In this route, the sequence of node values is $10 \cdots 1$, which can be explained as that $m$-cycle $\# 1$ is selected, $m$-cycle $\# 2$ not, $\ldots$, and $m$-cycle \#M is selected again.

Please note that in the above description, the cycles are ascending ordered according to their lengths from the root to leaf. Such an arrangement adds cycles to the cover solution in the increasing order of their lengths. But it is possible to get a lower-cost cover more quickly by adding in a cycle with higher length. A math example is $1+2+3+4>1+6$. Howerver, in the algorithm all cases are checked and compared in the algorithm. So the " $1+6$ " case would not be missed. Actually, sooner or later, the " $1+6$ " case will be checked and compared with " $1+2+3+4$ " case, and consequently will replace the later as an updated solution. Secondly, the impact of the order (ascending or descending) is only on the computing speed. The computing speed is not critical for offline algorithms. Finally, even we change the searching order to any other way, we have 
the same problem: in some cases we need to check the " $1+2+3+4$ " case sooner to accelerate the searching, but in vice versa for some other cases.

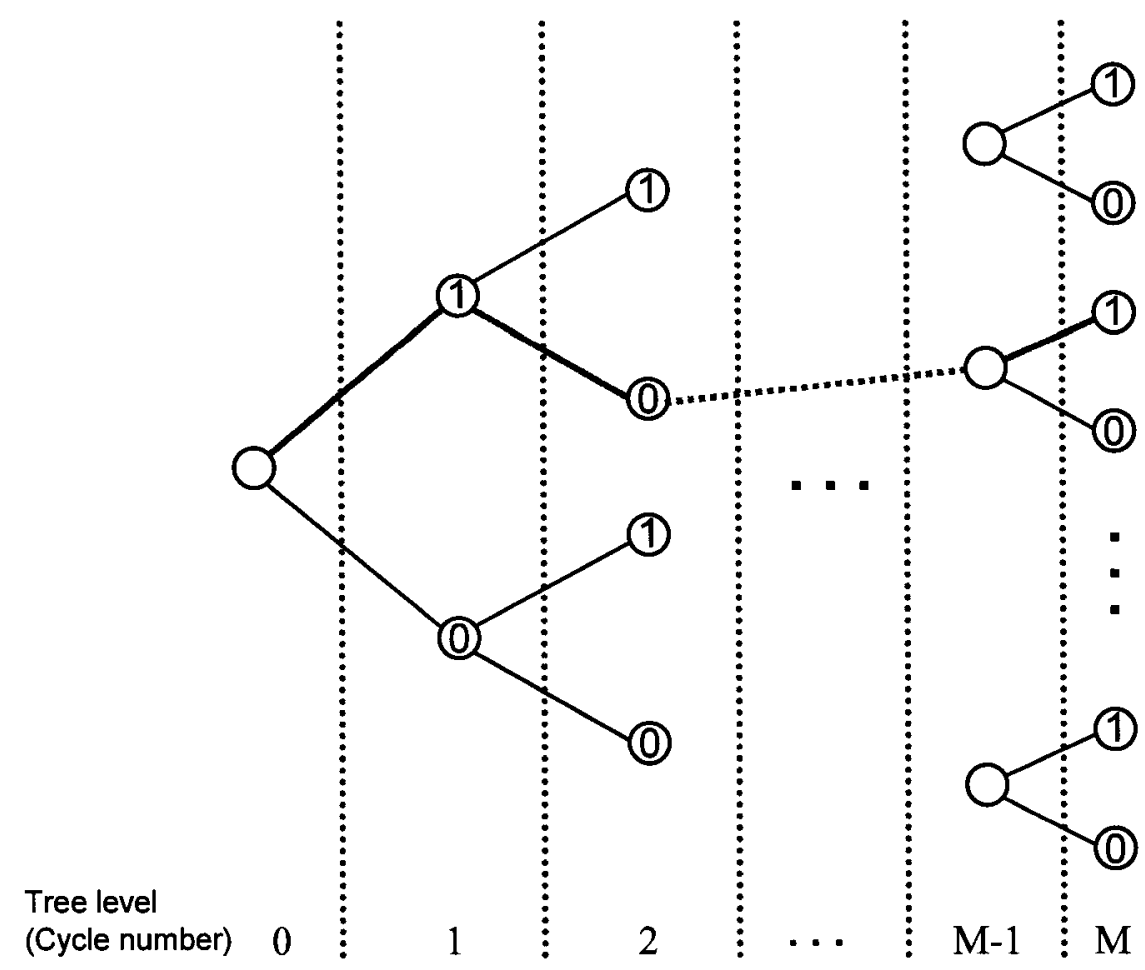

Figure 6.4 The full binary tree for $\mathrm{B} \& \mathrm{~B}$ and an example root-leaf route

Once a root-leaf route is determined, the link coverage, complete fault localization, and cost of the corresponding cycle combination can be checked and calculated immediately. If the link coverage and complete fault localization can be achieved, the combination of cycles is a feasible solution for the vCCCP and could be taken as a tentative solution.

By enumerating routes from the root to all leaves in the tree, checking their link coverage and complete fault localization, as well as comparing their cost, the optimum 
solution is theoretically guaranteed to be found.

To accelerate the searching procedure, once a feasible solution is found, it is set to be the tentative solution. The initial value of the cost of the tentative solution is set to be infinity for the convenience of software implementation. The B\&B algorithm calculates the cost of the current selected cycles at each node along the route from root to the current node. If the cost is larger than that of the tentative solution at a node, the rest of this route and all routes share the same partial path from the root to the current node can be pruned, i.e., the sub-tree from here can be pruned. The reason of such pruning relies on the fact that the cost increases monotonically along the route from the root to leaf. All cycles are sorted by length and they are put in the tree in the ascending order of cycle length from root to leaves. At each node, the algorithm also checks the link coverage and complete fault localization. If these two constraints are satisfied at the current node, the route from the root to the current node represents a feasible solution (not necessarily to be the optimal solution). If the cost of the current solution is better than that of the tentative solution, it updates the tentative solution with the current solution including its cost, prunes the sub-tree of the current node, and continues the B\&B searching, until all remainder routes in the binary tree are checked). The pruning operation would significantly speed up the $B \& B$ algorithm. In this way, the optimal solution could be quickly found by traversing all the root-leaf routes in the full binary tree.

The cost function for bound check includes both the cost of monitoring devices and the cost of reserved wavelength for monitoring in each link. Denote the cost of a monitor as $\alpha$ and the cost of a wavelength for link $e_{i}$ in an $\mathrm{AON}$ is $\beta_{i}$, where the value of $\beta_{i}$ depends on the number of available wavelengths in link $e_{i}$, the fiber length of link $e_{i}$, 
and any other user-defined factors. Based on such denotes and with the link-cycle associative bit, $a_{i j}$ (defined in equation 3.3.1 in Chapter 3.3), the cost function is calculated as:

$$
\alpha M+\sum_{i=1}^{L} \sum_{j=1}^{M} \beta_{i} a_{i j}
$$

\subsection{A descriptive example of solving vCCCP}

The two-phase B\&B algorithm described in Chapter 6.2 is applied to a simple example network $G(V, E)$ shown in Figure 6.5, to explain the algorithm in details.

There are 10 edges and 6 nodes in the network example. In the first phase (SPCC) of the two-phase B\&B algorithm, it builds shortest-path cycles for each edge-node combination. For example, for edge $(1,2)$, the shortest candidate cycles are generated as the following:

1) Temporarily remove edge $(1,2)$ from the network;

2) Given a node, e.g., node 3, find the shortest path $P_{31}$ and $P_{32}$ from node 3 to node $I$ and 2, respectively, in $G(V, E \backslash\{(1,2)\})$, build a cycle with the paths $P_{3 l}$, $P_{32}$ and edge $(1,2)$;

3) Repeat step 2 for all nodes in $V \backslash\{1,2\}$;

4) Repeat step 2-3 for all edges in E;

5) Sort cycles obtained at step 4 according to their lengths, delete the repeated and bad cycles (as defined in Chapter 6.5.1) and generate the candidate-cycle set; 
6) Check the existence of feasible solutions upon the candidate-cycle set by the procedure described in Chapter 6.5.2. And add extra cycles if necessary, insert the extra cycles into the candidate-cycle set and maintain the order of the set.

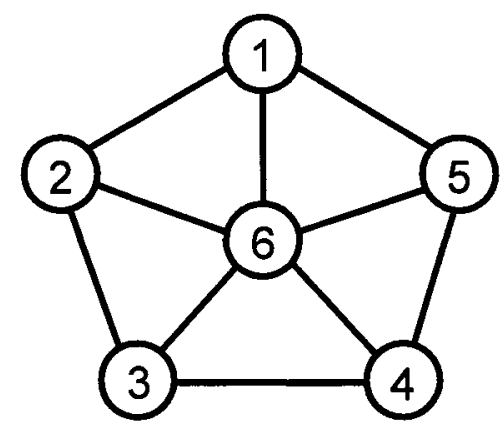

Figure 6.5 Example network $G(V, E)$ for the two-phase B\&B algorithm

The shortest paths and corresponding cycles for edge $(1,2)$ produced by SPCC are listed in Table 6.1. By repeat the procedure, cycles for all edges in $G(V, E)$ can be generated. Table C.1 given in Appendix C lists the results after sorting these cycles in order of cycle length (weight) and removing the repeated and bad cycles.

After the results listed in Table C.1 are ready, the algorithm then checks the existence of feasible solutions upon these cycles. According to the description in Chapter 6.5, a checking-alarm matrix similar to Figure 6.3 can be established as shown in Table C.2 in Appendix C. It can be immediately determined that link coverage and complete fault localization constraints are met upon the cycles listed in Table C.1, which implies at least one feasible solution can be established based on those cycles. 
Table 6.1 The shortest paths and corresponding cycles produced by SPCC for edge $(1,2)$ in the network example in Figure 6.5

\begin{tabular}{ccclc} 
Node & Shortest-path 1 & Shortest-path 2 & Cycle & Cycle length \\
\hline 3 & $1-6-3$ & $2-3$ & $1-6-3-2-1$ & 4 \\
4 & $1-5-4$ & $2-3-4$ & $1-5-4-3-2-1$ & 5 \\
5 & $1-5$ & $2-6-5$ & $1-5-6-2-1$ & 4 \\
6 & $1-6$ & $2-6$ & $1-6-2-1$ & 3 \\
\hline
\end{tabular}

Now the algorithm enters into the second phase: B\&B. Firstly a full binary tree with a null root node and 16 levels of other nodes is established, as shown in Figure 6.6. A value of either one or zero is assigned to each node, where the corresponding cycle is selected for the current solution in root-leaf routes searching when this value is one, and not selected when the value is zero.

In the construction of the tree, let the cycle closer to the root have smaller length. Also, let each node be the right-child of its parent-node if it has a value of 1 , and be leftchild with value zero (it is equivalent to set the left-child has the value one and right-child has value zero). The cycle length is the number of edges contained in the cycle and the cost of a solution (a set of cycles) is the total length of all cycles in it, plus the cost of monitors. Since the algorithm is searching for the minimum total-length solution in the tree, and the cycle closer to the root has smaller length, it searches root-leaf routes using the right-first rule at each node. The right-first rule checks the cycle with smaller length before ones with larger lengths. Thus it tries to reach a solution with smaller total length fast. 

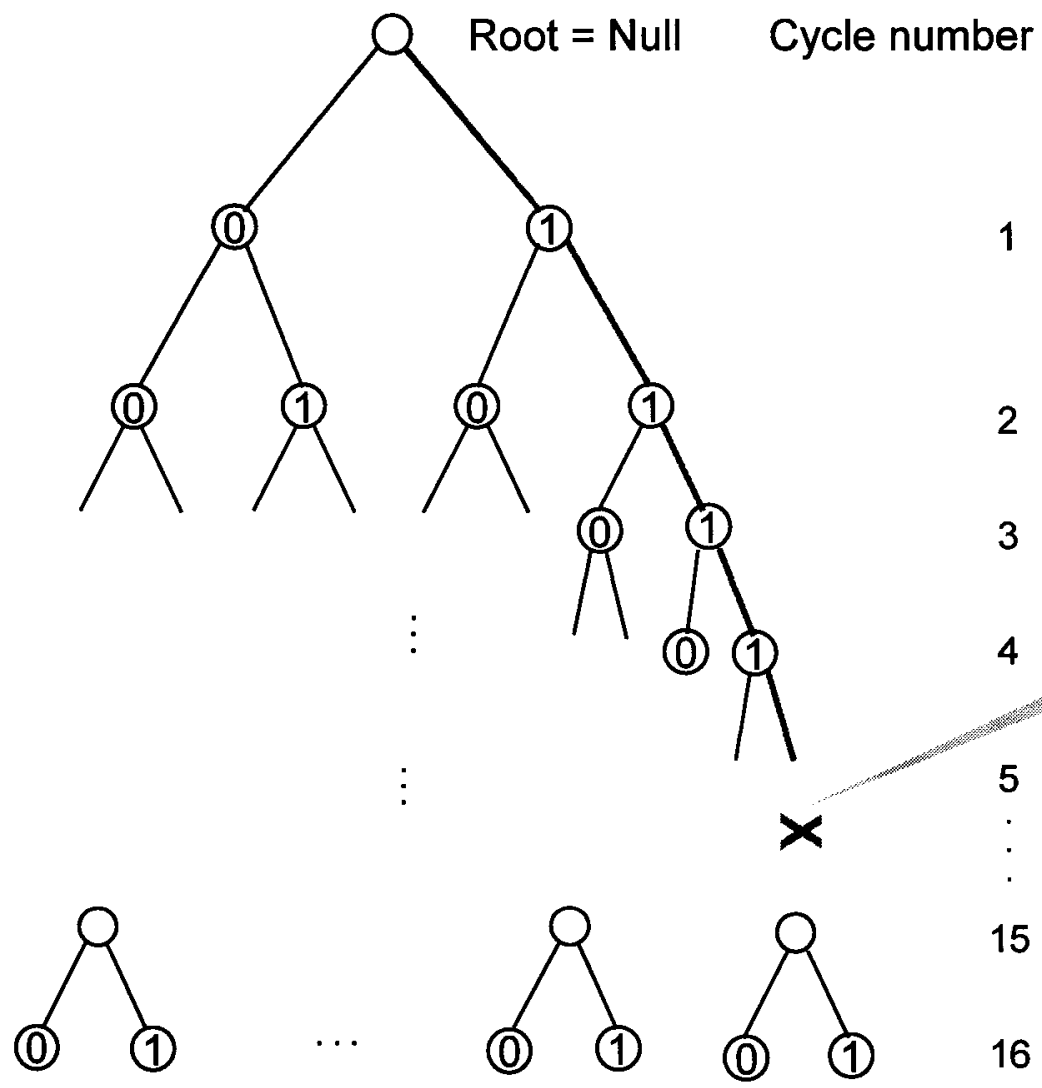

Figure 6.6 The full binary tree of B\&B for the network example in Figure 6.5

At the beginning of the algorithm, both the tentative and current solution are set to be null, their total-costs are set to be infinite. In the full binary tree (see Figure 6.6), the B\&B algorithm checks the link coverage and complete fault localization at each node (except for the root) in every root-leaf route. If the above constraints are not satisfied, the algorithm adds the right-child of the current node to the current solution. Otherwise, a feasible solution is achieved and its cost is compared with the tentative solution. The tentative solution will be updated by the current solution if the later one has smaller cost. For example, the B\&B algorithm checks the link coverage and complete fault localization along the root-leaf route shown in bond line. When it reaches the node corresponding to 
the fifth candidate cycle, a feasible solution is achieved. Since at this point the tentative solution is still null, so it is updated by this feasible solution with a cost of $3+3+3+3+3=15$. If the algorithm goes further to the level of leaves in the tree, even a feasible solution might be met, the cost of it will exceeds 15 and will be dropped. Therefore, the B\&B algorithm prunes the calculation for the whole sub-tree (both right and left sub-tree) of the current node (from the " $\mathrm{X}$ " sign in Figure 6.6), to accelerate the enumeration procedure of all root-leaf routes. The final solution is obtained by repeating the above procedure and is listed in Table 6.2.

Table 6.2 Final solution of the B\&B algorithm for the example in Figure 6.5

\begin{tabular}{lcccccccccc}
$m-$-cycle & $(1-2)$ & $(1-5)$ & $(1-6)$ & $(2-3)$ & $(2-6)$ & $(3-4)$ & $(3-6)$ & $(4-5)$ & $(4-6)$ & $(5-6)$ \\
$1-2-6-1$ & 1 & & 1 & & 1 & & & & & \\
$1-5-6-1$ & & 1 & 1 & & & & & & & 1 \\
$2-3-6-2$ & & & & 1 & 1 & & 1 & & & \\
$3-4-6-3$ & & & & & & 1 & 1 & & 1 & \\
$4-5-6-4$ & & & & & & & & 1 & 1 & 1 \\
\hline
\end{tabular}

\subsection{Performance Analysis}

To evaluate the performance of the vCCCP algorithm developed in Chapter 6.2, it is applied to four typical network examples listed in Chapter 3.3 (NSFNET, ARPA2, SmallNet, and Bellcore). The nodes of these topologies are re-labeled and shown in Figure 6.7. The vCCCP algorithm is developed for network topologies that satisfy the condition of complete fault localization.Some network examples satisfy such conditions, 
e.g., SmallNet, but some others not, e.g., NSFNET. Before the vCCCP algorithm is applied to those topologies not satisfying that condition, some transform are needed for them.

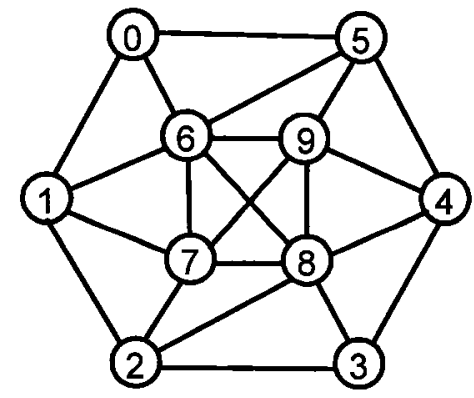

(a) SmallNet

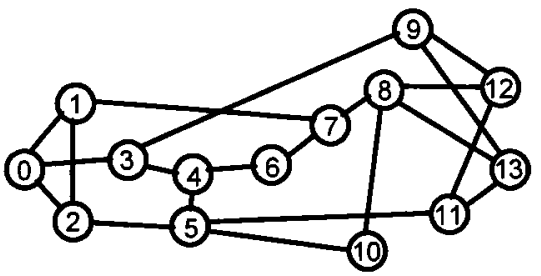

(c) NSFNET

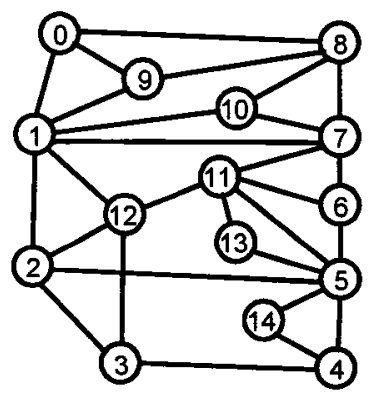

(b) Bellcore

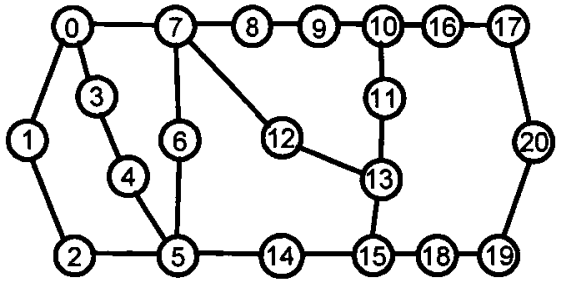

(d) APAR2

Figure 6.7 Network Examples for evaluating the B\&B vCCCP algorithm

Chapter 5 showed that the degree- 2 chain is the key to violate the conditions. Therefore, a straightforward transform is to shrink all degree- 2 chains in a topology into a single edge. For links within a degree- 2 chain, no matter how to choose and expand the $m$-cycle set, they are not separable (please see Chapter 5.1). Thus for $s$ edges within the same degree-2 chain, one monitor-per-edge policy can be applied for $s-1$ edges of them (in total $s-1$ extra monitors are needed), to achieve the complete fault localization.

Among the four network examples, the SmallNet satisfies the conditions and needs no transform. For the other three, removed degree- 2 chains and edges need extra 
monitors are shown in Figures $6.8-6.10$. Please note that in Figure 6.10 for the network ARPA2, after the first round of chain removing, some new chains occur. So it needs to repeat the removal operation until there is no degree- 2 chain in the topology. It is clear that for such a network with parse nodes, there are too many un-separable edges and thus the performance of $m$-cycle based FDL might not achieve significant savings comparing to the monitor-per-link method.
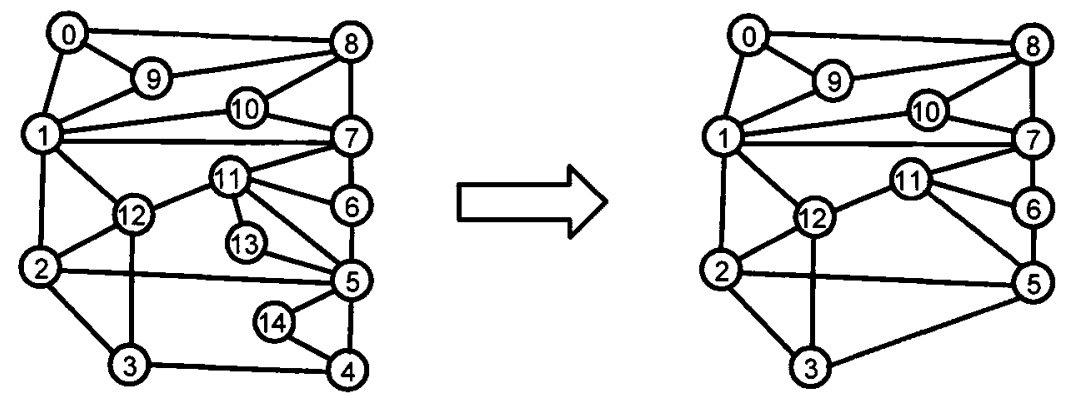

Removed edges: $3-4,4-5,4-14,5-14 \rightarrow 3-5 ; \quad 5-13,11-13,5-11 \rightarrow 5-11$

Figure 6.8 Remove degree-2 chains in Bellcore
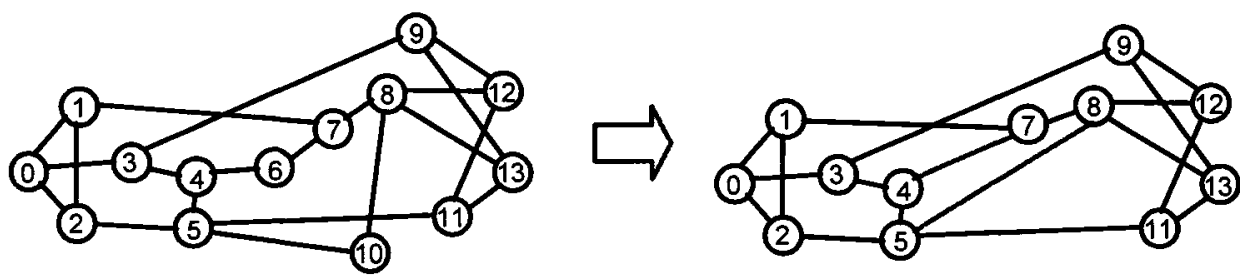

Removed edges: $5-10,10-8 \rightarrow 5-8 ; 4-6,6-7 \rightarrow 4-7$

Figure 6.9 Remove degree-2 chains in NSFNET 

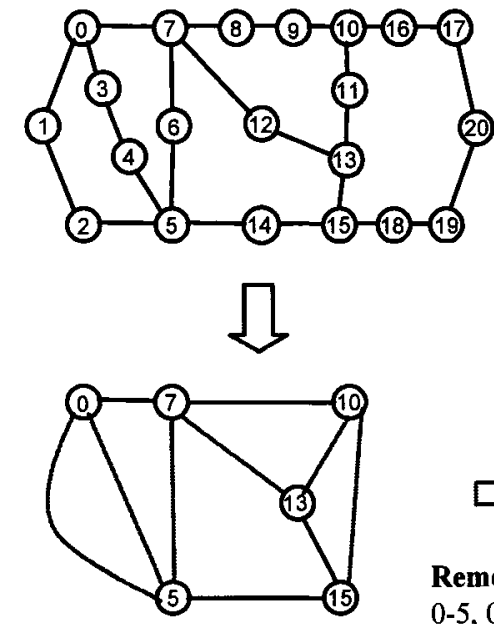

Removed edges (step 1):

$0-1,1-2,2-5 \rightarrow 0-5$

$0-3,3-4,4-5 \rightarrow 0-5$ (duplicated)

$5-6,6-7 \rightarrow 5-7$

$7-12,12-13 \rightarrow 7-13$

$5-14,14-15 \rightarrow 5-15$

$7-8,8-9,9-10 \rightarrow 7-10$

$10-11,11-13 \rightarrow 10-13$

$10-16,16-17,17-20,20-19,19-18,18-15 \rightarrow 10-15$
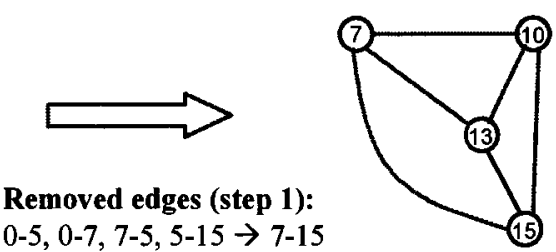

Figure 6.10 Remove degree- 2 chains in ARPA2

\subsubsection{Localization degree, wavelength overhead, and cost reduction}

Tables C. 3 - C.6 given in Appendix C list the final results (optimal solutions) for network examples without degree-2 chain obtained by vCCCP B\&B algorithm. Please note that there exist multiple solutions for a single vCCCP and the proposed B\&B algorithm can find all solutions with the minimum cost. Cycles are denoted as the sequence of node numbers within them. All node numbers in the cycles are the node labels in the corresponding graphs after removing degree- 2 chains.

To evaluate the algorithm performance, the above results are compared with the $m$ cycles produced by previous algorithms (HDFS, SPEM, and HST, see Chapter 4). Statistical data are summarized in Tables 6.3 and 6.4. In these tables, $L D$ is the localization degree, $M$ is the number of cycles in a solution, and $M^{\prime}$ is the extra number of monitors needed for achieving complete fault localization. The wavelength cost is the total number of wavelengths reserved for $m$-cycles, i.e., the number of total edges in all $m$-cycles in the solution. $A_{\max }$ and $A_{\text {avg }}$ is the maximum and average number of 
reserved wavelengths for monitoring in a single link, respectively. $\Lambda_{\text {avg }}$ measures the average overhead of wavelengths due to MFLA. $\Lambda_{\max }$ is important due to the fact that it gives a bench value, while the MFLA is not applicable if this value is close to or even larger than the number of total available wavelengths in the link.

Table 6.3 shows the raw output directly obtained from the $m$-cycle construction algorithms for comparing. Based on these $m$-cycles, the complete fault localization is not always achievable for the original topology (before remove chains). With some extra monitors added according to the rule described at the beginning of this sub-chapter, a fairly comparison is given in Table 6.4, where the complete fault localization is achieved in all cases.

The comparison shows that the B\&B algorithm produces smaller numbers of $m$ cycles, and less wavelength-cost than other algorithms in all examples, under the fairly comparison that achieves the complete fault localization. The main reason of such an improvement can be attributed to the fact that the two-phase vCCCP algorithm generates more comprehensive candidate cycles, and more importantly, the shortest cycles for all edge-node combinations are included in the candidate-cycle set for a given graph. Based on a systematic searching (B\&B), the feasible and optimal solutions based on the comprehensive candidate-cycle set are guaranteed, as discussed in Chapter 6.2.2. Further observation of these tables discloses that the more complex is the network example (with higher average node degree, refer to Chapter 4.5 ), the more savings can be achieved by $B \& B$ algorithm comparing to the others. Thus the $B \& B$ algorithm is suitable for complex networks, or simply say, scalable in this sense. 
Table 6.3 $M$-cycle construction comparison: raw results in network examples

\begin{tabular}{|c|c|c|c|c|c|c|}
\hline Network & Alg. & LD & $\mathbf{M}$ & Wavelength Cost & $\Lambda_{\text {avg }}$ & $A_{\max }$ \\
\hline \multirow{4}{*}{ SmallNet } & B\&B & 1.00 & 12 & 36 & 1.64 & 3 \\
\hline & HST & 1.00 & 13 & 43 & 1.95 & 6 \\
\hline & HDFS & 1.47 & 8 & 34 & 1.55 & 3 \\
\hline & SPEM & 3.67 & 4 & 26 & 1.18 & 2 \\
\hline \multirow{4}{*}{ Bellcore } & B\&B & 1.21 & 11 & 38 & 1.36 & 2 \\
\hline & HST & 1.08 & 14 & 55 & 1.96 & 8 \\
\hline & HDFS & 2.15 & 6 & 40 & 1.43 & 3 \\
\hline & SPEM & 3.67 & 5 & 32 & 1.14 & 2 \\
\hline \multirow{4}{*}{ NSFNET } & B\&B & 1.11 & 8 & 35 & 1.67 & 4 \\
\hline & HST & 1.11 & 8 & 40 & 1.90 & 5 \\
\hline & HDFS & 1.50 & 6 & 33 & 1.57 & 3 \\
\hline & SPEM & 3.00 & 4 & 26 & 1.24 & 2 \\
\hline \multirow{4}{*}{ ARPA2 } & B\&B & 2.17 & 3 & 27 & 1.64 & 2 \\
\hline & HST & 2.50 & 5 & 40 & 1.95 & 6 \\
\hline & HDFS & 3.13 & 4 & 34 & 1.55 & 3 \\
\hline & SPEM & 5.00 & 4 & 30 & 1.18 & 2 \\
\hline
\end{tabular}

Table 6.4 M-cycle construction comparison: results for achieving complete fault localization in network examples

\begin{tabular}{c|l|c|c|c|c|c|c}
\hline Network & Alg. & $\mathbf{M}$ & $\mathbf{M}^{\prime}$ & $\mathbf{M}+\mathbf{M}^{\prime}$ & Wavelength Cost & $A_{\text {avg }}$ & $A_{\max }$ \\
\hline \multirow{5}{*}{ SmallNet } & B\&B & 12 & $/$ & 12 & 36 & 1.64 & 3 \\
\cline { 2 - 9 } & HST & 13 & $/$ & 13 & 43 & 1.95 & 6 \\
\cline { 2 - 9 } & HDFS & 8 & 7 & 15 & 41 & 1.86 & 3 \\
\hline & SPEM & 4 & 16 & 20 & 42 & 1.91 & 2 \\
\hline \multirow{5}{*}{ Bellcore } & B\&B & 11 & 4 & 15 & 42 & 1.50 & 2 \\
\cline { 2 - 9 } & HST & 14 & 2 & 16 & 57 & 2.04 & 8 \\
\cline { 2 - 9 } & HDFS & 6 & 15 & 21 & 55 & 1.96 & 3 \\
\hline & SPEM & 5 & 21 & 26 & 53 & 1.89 & 2 \\
\hline \multirow{5}{*}{ NSFNET } & B\&B & 8 & 2 & 10 & 37 & 1.76 & 5 \\
\cline { 2 - 9 } & HST & 8 & 2 & 10 & 42 & 2.00 & 5 \\
\hline & HDFS & 6 & 7 & 13 & 40 & 1.90 & 3 \\
\hline & SPEM & 4 & 15 & 19 & 41 & 1.95 & 2 \\
\hline \multirow{5}{*}{ ARPA2 } & B\&B & 3 & 17 & 20 & 47 & 1.64 & 3 \\
\cline { 2 - 9 } & HST & 5 & 15 & 20 & 55 & 1.95 & 6 \\
\cline { 2 - 9 } & HDFS & 4 & 16 & 20 & 50 & 1.86 & 3 \\
\cline { 2 - 9 } & SPEM & 4 & 18 & 22 & 48 & 1.91 & 2 \\
\hline
\end{tabular}


With a closer observation at the detail of cost function check in the B\&B algorithm, readers could find that it is flexible to set the cost function, e.g., set different weight for edges in the network, to avoid some special network link with high management-expense, or put favorites on links with lower expenses.

\subsubsection{Impacts of $\alpha$ and $\beta$}

In the above simulation of $\mathrm{B} \& \mathrm{~B}$ algorithm, as defined in Chapter 6.1, parameter $\alpha$ is the cost coefficient of a monitor. $\beta$ actually represents for a set of cost coefficients of wavelengths, $\beta_{e}$, which is the cost of one wavelength within link $e$. For the simplicity of the simulation, the cost coefficients are set to be $\beta_{1}=\beta_{2}=\cdots=\beta_{L}=\beta$ and $\alpha=\beta=1$. Such setting is applicable for the case that all links in an AON have the same number of available wavelengths. If numbers of available wavelengths in network links are various, the parameter $\beta_{e}$ can be set to different values, which is proportional to the number of available wavelengths in link $e$, to incorporate the impacts of this factor. Actually, for the purposes used here, a single parameter which is the $\beta / \alpha$ relative value, or equivalently $\beta_{e} / \alpha$, can be defined to replace the parameter pair $\alpha$ and $\beta$. The only reason that two parameters are defined here is for the simplicity of the simulation programming.

For various values of parameters $\alpha$ and $\beta$, upon the 4 network examples from the real world, results of conducted simulations show that the B\&B algorithm obtains the same $m$-cycles with various $\alpha$ and $\beta$. Even with some extreme cases, e.g., $\alpha=1, \beta=0$ when consider only the cost for monitoring devices but without the consideration of 
wavelength, or $\alpha=0, \beta=1$ when consider only the wavelength cost but without the cost of monitors, the B\&B algorithm produce the same results upon the four typical network examples. It shows that, in the given examples, when the cost of wavelengths is optimized, the number of cycles is also minimized at the same time, or vice versa. Although some examples could be found, theoretically, that the wavelength cost is optimized but the cycle number is not, or vice versa, the simulation partially shows that in the real world, the impacts of $\alpha$ and $\beta$ are not very critical for the optimization results for the given network examples.

\subsubsection{Complexity analysis for the vCCCP solving algorithm}

As discussed for HDFS, SPEM and HST, the time complexities of the two-phase vCCCP algorithm can be estimated, although the MFDL is an off-line algorithm and the time complexity is not critical. For the two-phase vCCCP algorithm, the complexity of Dijkstra algorithm (shortest-path) is $D=O\left(V^{2}\right)$, so the complexity for the first phase (generates candidate cycles) is $O\left(L^{*}\left[2 *(V-2)^{*} D\right]\right) \sim O\left(L V^{3}\right)$. The B\&B algorithm does not lower the worst-case time complexity of the searching, which is exponential. According to the SPCC procedure, the candidate cycle number is less than $L(V-2)$. Therefore, the B\&B complexity for worst case is still exponential, $O\left(2^{M}\right)$ (while $\mathrm{M}$ is the number of $m$-cycles). But in practice the pruning significantly reduces the average running time up to $90 \%$ [134], as pointed out in Chapter 4.2. The comparison of complexity analysis for worst case is summarized in Table 6.5 . 
Table 6.5. Comparison of time complexity for four $\mathrm{m}$-cycle construction algorithms

\begin{tabular}{c|c|c|c|c}
\hline Algorithm & HDFS & SPEM & HST & vCCCP \\
\hline Complexity & $O\left(L V+L^{2}\right)$ & $O\left(V^{2}+L\right)$ & $O\left(V^{2}+V L+L^{2}\right)$ & $O\left(2^{M}\right)$ \\
\hline
\end{tabular}

\subsection{Empirical Study for vCCCP: Randomly Generated}

\section{Network Examples}

To make the performance evaluation more statistically meaningful, the two-phase vCCCP algorithm is applied to some randomly generated network examples. Such network examples are generated using BRITE, developed by a research group at department of Computer Science, Boston University, and with their kind courtesy [132].

The network examples randomly generated using BRITE are listed in Tables D.1, D.3, D.5 and D.7 in Appendix D, in the format of from- and to-node numbers. Please note in all examples degree- 2 chains have already been removed before applying the two-phase vCCCP algorithm and thus they all satisfy the complete fault localization condition proven in Chapter 5.1. $M$-cycles obtained from those random network examples using the two-phase vCCCP algorithm are listed in Tables D.2, D.4, D.6 and D.8 in Appendix D. Those results are summarized and the cost reduction is calculated in Table 6.6 for comparison. Again, the results show the significant savings of expensive optical monitors (equivalent to $m$-cycle numbers) comparing to monitor-per-link case, which is consistent with the performance analysis for the four examples from the real world. 
Table 6.6. Summary of MFLA results on randomly generated network examples

\begin{tabular}{c|c|c|c|c|c|c}
\hline $\begin{array}{c}\text { Network } \\
\text { Example }\end{array}$ & $\begin{array}{c}\text { Node } \\
\text { Number }\end{array}$ & $\begin{array}{c}\text { Link } \\
\text { Number }\end{array}$ & $\begin{array}{c}\text { Average } \\
\text { Node } \\
\text { Degree }\end{array}$ & $\begin{array}{c}m \text {-cycle } \\
\text { number in } \\
\text { solution }\end{array}$ & $\begin{array}{c}\text { Wavelength } \\
\text { number in } \\
\text { solution }\end{array}$ & $\begin{array}{c}\text { Cost } \\
\text { reduction }\end{array}$ \\
\hline 1 & 13 & 20 & 3.1 & 7 & 35 & $65.0 \%$ \\
\hline 2 & 18 & 37 & 4.1 & 25 & 93 & $32.4 \%$ \\
\hline 3 & 21 & 37 & 3.5 & 27 & 123 & $27.0 \%$ \\
\hline 4 & 30 & 61 & 4.1 & 46 & 205 & $25.0 \%$ \\
\hline
\end{tabular}

$-129-$ 


\section{Chapter 7}

\section{Implementation of MFDL}

$\mathrm{T}$

THE previous chapters of this thesis introduced a new $m$-cycle based FDL (MFDL) approach for mesh AONs. The performances are also evaluated through applying it to some typical network examples. This chapter discusses the application and implementation of such MFDL schemes.

The implementation of MFDL includes two major parts: alarm delivery from the monitoring device in an $m$-cycle to the network management station (NMS), and the faulty-source notification from the NMS to related network elements. The alarm delivery and faulty-source notification messages are transported in control channels within the control plane of AONs (usually in a TCP/IP network separated to the data plane, please refer to Chapter 2.1).

Generally, the MFDL can be implemented either in the centralized or the distributed fashion. In the distributed implementation, each network node is flexible to manage its own environments and has the knowledge of the service health of lightpaths gone through it. There is greater redundancy in distributed implementation and thus there is less reliance on a single-point centralized NMS, which could be interrupt and be unavailable upon certain network failures. However, alarms might be generated in multiple $m$-cycles for a single network fault and such alarms need various time latencies to flood to the 
whole network. It is more difficult to synchronize those alarms for fault localization because in the centralized implementation there exists at least a central clock in the system for the purpose of synchronization. The complexity of synchronization may consequently slow down the fault localization speed. Furthermore, the flooding of redundant alarms to the whole network for a single failure puts more traffic overhead to the limited bandwidth in the control plane.

On the other hand, the centralized fault localization methods are more efficient than their distributed counterparts and bring less traffic overhead. It provides a single view of the lightpath health for the whole AON through central consoles and reporting. The operations and management expenses are reduced through consolidation of management tools and human administrators in the network operations center (NOC). Additionally, NMS failure is rare under the current technologies and environments so that the singlepoint failure of the central server is not a problem. According to the report published by MicroSoft $^{\circledR}$ in [120], 30\% of the 500 largest Global 2000 enterprises use a centralized architecture for their network OAM\&P and $60 \%$ of the 500 largest enterprises will attain centralized management architecture by 2006 . Based on the above analyses, the discussion of MFDL implementation is focused on the centralized fashion in this thesis.

The simple network management protocol (SNMP), which was defined in 1988 and was approved by the Internet Activities Board as an Internet Standard in 1990, has received wide acceptance [121-122]. For example, SNMP v1 and v2 have been already implemented in one of the most popular personal computer operation systems, Microsoft Windows 2000. Therefore, SNMP plays a critical role in modern network management, especially for alarm monitoring. Consequently it is easy and cost-effective to deploy 
SNMP for alarm delivery in MFDL implementation.

\subsection{SNMP Introduction}

SNMP is an application-layer protocol for managing TCP/IP based networks, which runs over User Datagram Protocol (UDP) and in turn, over Internet Protocol (IP). The latest version of SNMP definition can be found in [122].

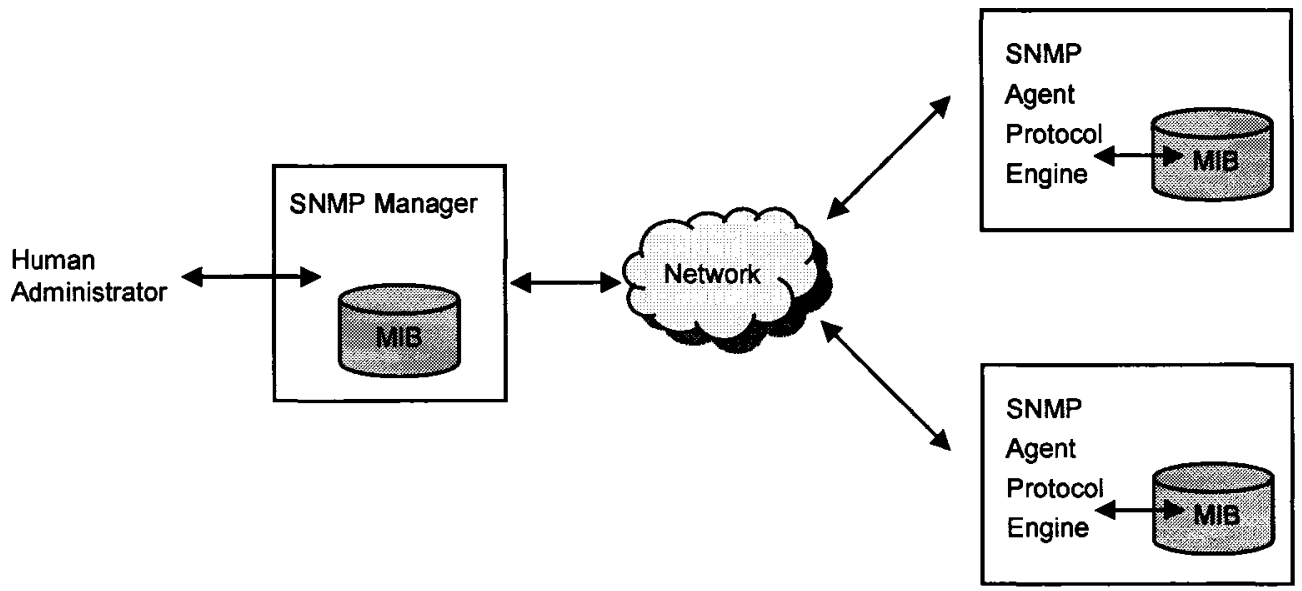

Figure 7.1 Architecture of the simple network management protocol (SNMP)

SNMP is based on two primary elements: a manager and agents. The manager is the interface between the human administrator and the network management system (NMS). Agents are the interface between the SNMP manager and the actual devices being managed. The entities can be manipulated by SNMP are defined as managed objects. Managed objects include hardware, configuration parameters, performance statistics, and 
so on, which are residing in the managed devices like bridges, hubs, routers, and network servers, etc. Managed objects are arranged in a virtual information database that is known as the management information base (MIB). The SNMP manager exchanges information with agents via MIBs using a small set of commands and responses. The architecture of the SNMP is shown in Figure 7.1.

\subsubsection{Structure of Management Information and MIB}

The SNMP manages specific objects and each object has specific characteristics. The structure of management information (SMI) is defined in separate Internet RFCs [123124] and it describes how management information is referenced and stored. The SMI states that every object or characteristic must have a name, the syntax, and an encoding. The name is a unique object identifier (OID), which uniquely identifies the object in the SNMP system. The syntax defines the data type of the variables for objects or characteristics. The Abstract Syntax Notation One (ASN.1) [125-128] is deployed for the SNMP syntax. The encoding gives how to serialize the information associated with objects for transmission.

Management information base (MIB) is a set of definitions for object characteristics within the managed devices. Each managed device keeps a database of values for each definition in the MIB. The MIB serves as a data dictionary or codebook for assembling and explaining SNMP messages. The latest Internet MIB (MIB-II) is given in the Request for Comments (RFC) 1213 of the Internet Engineering Task Force (IETF) [129].

The object names, universal unambiguous OIDs for arbitrary objects, can be achieved via a hierarchical tree shown in Figure 7.2. In the tree each child node is assigned with a 
non-negative integer value, for which is unique among all nodes that have the same parent node. Child nodes could have subordinates (further child sub-trees) where the numbering scheme is applied recursively. Then the OID (or name) of an object is the sequence of non-negative integers obtained in traversing the tree to the required node. For example, the Cisco router OID is 1.3.6.1.4.1.9.1.1.

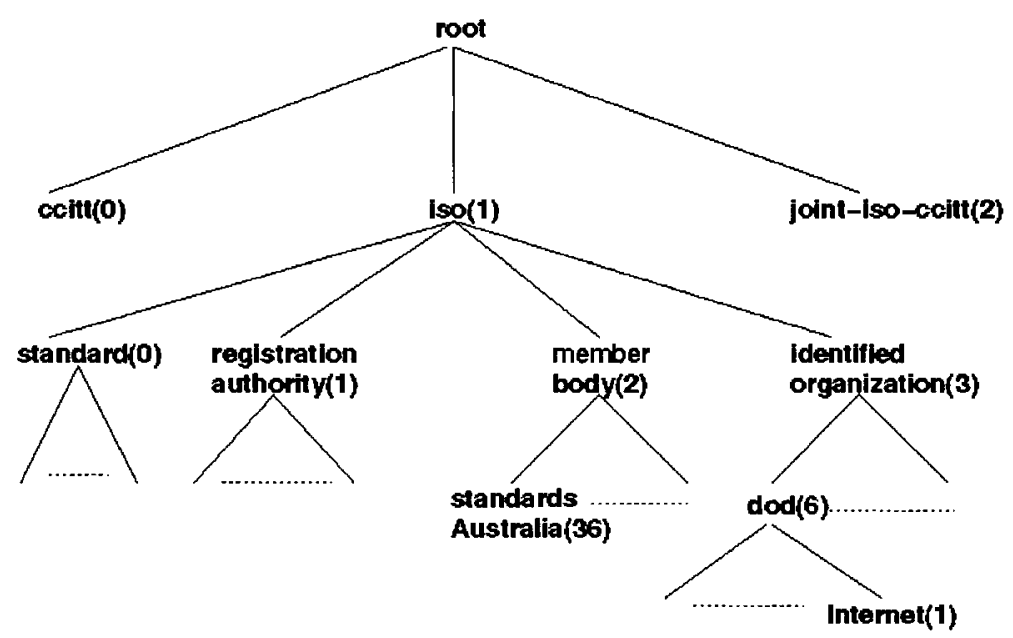

Figure 7.2 The hierarchical OID tree in the MIB

\subsubsection{SNMP commands}

The SNMP command set provides five primitive operations for communications between the manager and an agent:

- GetRequest: allows the manager to retrieve the information for a single or 
multiple object variables.

- GetNextRequest: is the same as the "Get" operation but it traverses down a table within a MIB. It is designed to retrieve the OID and value of the MIB instance that comes after the one asked for.

- GetResponse: the agent sends the "GetResponse" message to the manager upon receiving a "GetRequest" or "GetNextRequest" message. This message reports either the requested information to the manager, or an error indication for the reason that the request cannot be processed.

- SetRequest: allows the manager to establish or change the value of a single object variable.

- Trap: allows the agent to spontaneously notify the manager of an event, which is defined as the occurrence of conditions such as the threshold exceeds a predetermined value.

The SNMP manager issues most SNMP messages (Get, Get Next, and Set). The only message can be initiated by an agent is the Trap message. It allows an agent to notify the SNMP manager as soon as an alarm condition occurs, instead of waiting for the SNMP manager to poll.

SNMP assumes that communication paths for the transmission of commands and responses are connectionless. The primary protocols for SNMP implementations are UDP and IP in the real world, although SNMP is actually defined transport independently and can be implemented in other transports, e.g., ATM and X.25 protocols.

The simplicity of commands and responses, as well as the connectionless 
communications, produce a degree of robustness and have led to a widespread use.

\subsubsection{SNMP message types and formats}

Each SNMP message contains the version number, a community string, and one or more SNMP protocol data units (PDU). A community is defined by a community name, which is an OctetString with 0 to 255 octets in length. Each SNMP managed object belongs to one and only one community, while the NMS might belong to multiple communities. There are three kinds of community strings for SNMP messages: READONLY, READ-WRITE, and TRAP. For a GetRequest or GetNextRequest to the SNMP agent with READ-ONLY community string, if the agent is using the same read-only string it will process the request, otherwise it will deny the request. If a MIB object has an ACCESS value of read-write, then a Set PDU can change the value of that object with the correct read-write community string. A TRAP community string allows administrators to cluster network entities into communities.

SNMP defines two different PDU formats, which are shown in Figure 7.3. Each SNMP PDU except trap consists of:

- PDU type

- Request ID: request sequence number

- Error Status: zero for no error otherwise one of a small set

- Error Index: zero for no error otherwise the index of OID in the PDU that causes the error

- Variable Bindings: list of OIDs and their values. Null for GetRequest and GetNextRequest 


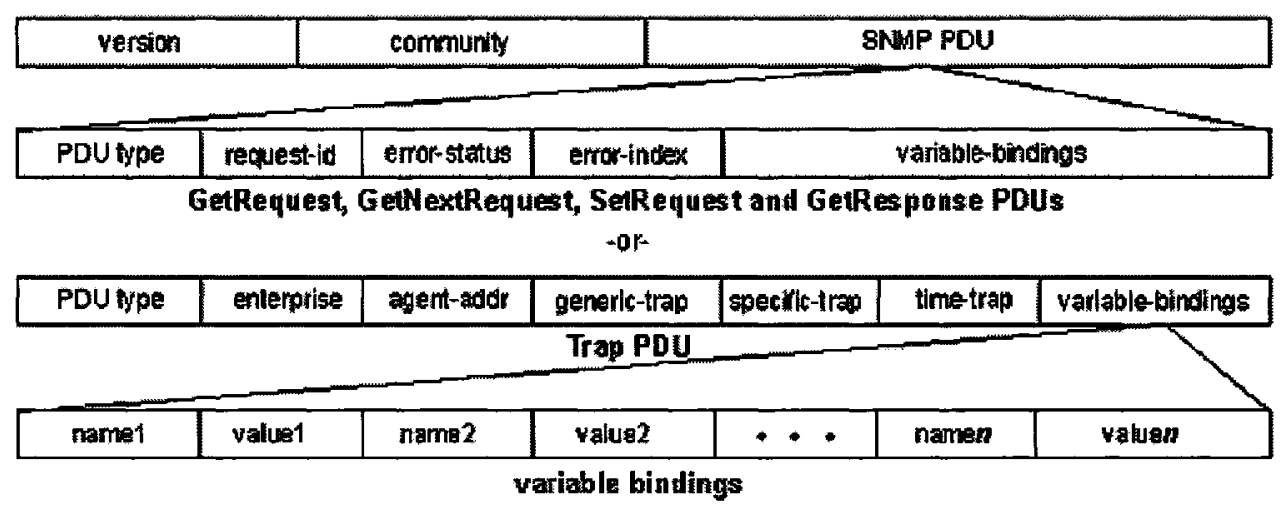

Figure 7.3 SNMP message formats

The trap PDU consists of:

- PDU type

- Enterprise: the type of the object causing the trap

- Agent Address: IP address of the agent that sent the trap

- Generic Trap ID: common standard traps

- Specific Trap ID: proprietary or enterprise trap

- Time stamp: when trap occurred in time ticks

- Variable Bindings: a list of OIDs and values

\subsection{Customization of SNMP for MFDL application}

As described in Chapter 7.1, the SNMP TRAP message can be immediately used for alarm delivery in MFDL. The alarm is transport from the monitoring device in an $m$ -

$-137-$ 
cycle to the NMS, through the control plane (see Chapter 2.1) of the given AON. The control plane is assumed to be reliable and connected via TCP/IP and an SNMP instance is running upon it.

\subsubsection{Register network alarms in the MIB tree}

Before applying the SNMP TRAP to alarm delivery for MFDL, an object characteristic, alarm indicator, should be defined and registered in the hierarchical MIB tree for each monitoring device. The alarm indicator has a binary value that is defined as below for each monitoring device in an $m$-cycle:

$$
\text { Alarm Indicator }= \begin{cases}1 & \text { An alarm is generated in the } m-\text { cycle } \\ 0 & \text { Otherwise }\end{cases}
$$

One more object characteristic, link status, is defined within each node in an AON for every link connected to this node. The link status gives the service health of the link and its value is binary as below:

$$
\text { Link Status }= \begin{cases}1 & \text { fault occurs along the link } \\ 0 & \text { Otherwise }\end{cases}
$$

In the hierarchical MIB tree, the enterprise sub-tree (private) consists of MIBs of proprietary objects (sub-tree registered with Internet Assigned Numbers Authority, as in Figure 7.4. The newly defined AlarmIndicator can be registered under the enterprise subtree for each kind of monitoring device, where the corresponding OIDs have the format of 1.3.6.1.4.1.x.y.

Once a network fault occurs, the agent residing in the corresponding monitoring device sets the AlarmIndicator and sends a TRAP message including the AlarmIndicator to the manager, to notify the manager the device's change in operational status. As 
indicated in Figure 7.3, A TRAP PDU contains a sequence of object name-value pairs. Names and values could be any parameters for fault detection in AONs described in Chapter 2.1, e.g., optical power, OSNR, BER, and so on, depending on the type of monitors assigned into $m$-cycles. Once the alarms are sent to the NMS, the fault localization algorithm generates a real-time alarm code and look up the code in a preestablished table to locate the faulty source (see Chapter 3.3 for the detailed procedure).

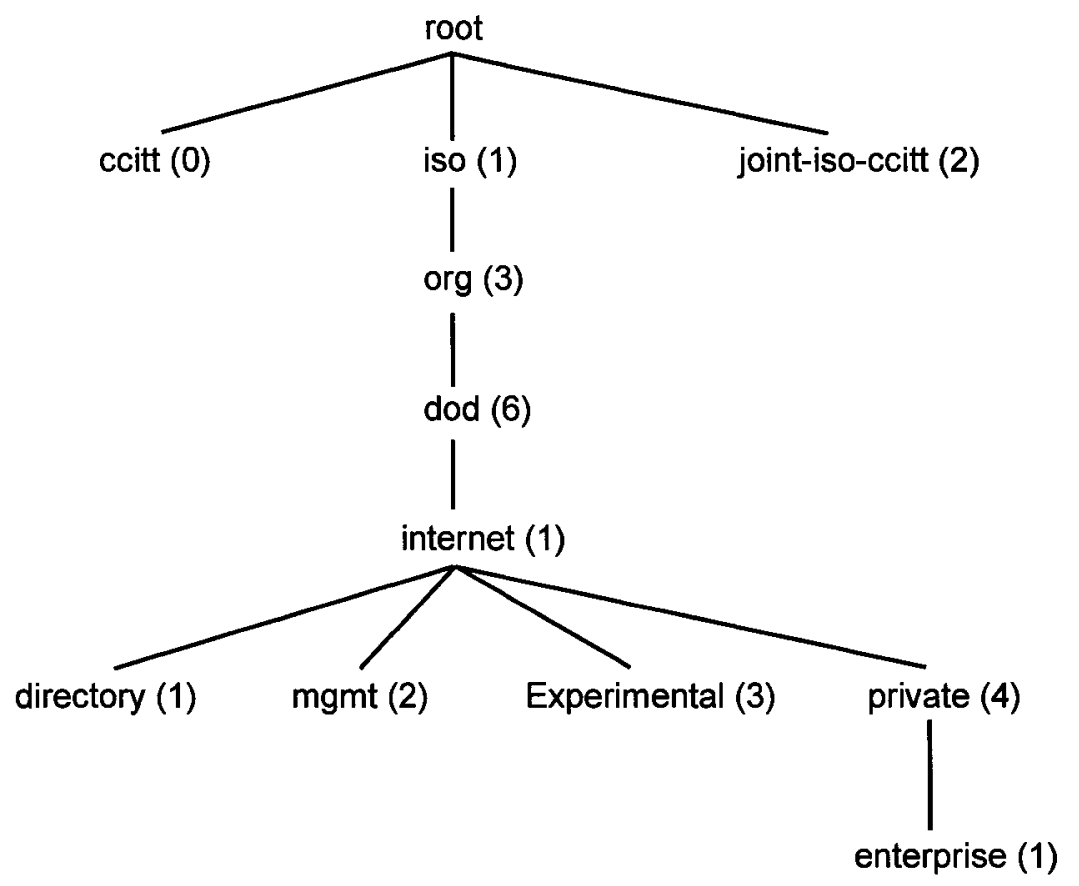

Figure 7.4 The enterprise sub-tree (private) in the MIB tree

\subsubsection{Alarm synchronization}

SNMP Trap command sends asynchronous notification, so alarms triggered by the same network fault but from different $m$-cycles may experience various transportation latency when they arrive in the NMS. So synchronization of alarms from different $m$ - 
cycles must be taken into consider. Since each TRAP message contains a time-stamp field, a short time period, called SuppressInterval, can be defined for alarm synchronization. If the time difference between the time-stamp in two TRAP-massages is smaller than the SuppressInterval, the two corresponding alarms are considered as being triggered by the same network faults. Otherwise, the NMS treats them as alarms for two different network faults. This is defined in accordance with the assumption of "singlefailure" scenario: in a certain short time period, only one network fault is possible to occur.

\subsubsection{Faulty-Source Notification in MFDL}

The faulty-source notification process is invoked once the faulty source is localized for a real-time alarm code. The notification message, the binary value of the link status (be one for a faulty link, see equation 7.2.2), is carried in the SNMP SET message and broadcast to all nodes in the given AON. The transmission of the notification is kept in the control plane. The notification recipients that are in charge of lightpaths containing the faulty source will invoke the residing protection/restoration schemes. For example, if the destination-initiating path restoration protocol [130] is assigned in the AON for dynamic fault restoration, and a network fault is localized to link $e$ (let the two endpoints of link $e$ are nodes $n_{1}$ and $n_{2}$ ), then the status values for link $e$ in $n_{1}$ and $n_{2}$ are updated to be one. Then $n_{1}$ and $n_{2}$ invoke the restoration protocol and shift the traffic to the backup lightpaths from the lightpaths that pass though link $e$. In the meanwhile, the information of this link is updated in all network nodes and could be used for any further lightpath provisioning (routing and wavelength assignment, RWA). 
Once the network fault is fixed by either human intervention or automatic procedure, the status of the faulty link is set to be zero again and the updated status value is sent to the NMS via SNMP TRAP message again. The NMS then broadcast the updated link status to the whole $\mathrm{AON}$ and complete the reversion process.

$-141-$ 


\section{Chapter 8}

\section{Conclusions and Future Work}

$\mathrm{I}$

$\mathrm{N}$ this thesis a novel fault detection and localization (FDL) approach was proposed

for mesh AONs. This approach decomposed an AON topology into cycles that form a cycle cover for the given network. Such cycles with assigned network monitors and supervisory channels within them were defined as monitoring-cycles ( $m$-cycles).

The work in this thesis showed the feasibility of $m$-cycle based FDL approaches in theory based on the well-known cycle double cover conjecture, followed by the proposal of an $m$-cycle based fault localization algorithm (MFLA). To evaluate the performances of the $m$-cycle based FDL approach, a group of evaluation metrics were introduced, including the fault localization degree, wavelength overhead, and cost reduction over the monitor-per-link method.

To find appropriate $m$-cycles in the topology of a given mesh AON, three $m$-cycle construction algorithms were proposed: the heuristic depth first searching (HDFS), the shortest-path Eulerian matching (SPEM), and the heuristic spanning-tree (HST) based algorithms. These $m$-cycle construction algorithms, together with the fault localization algorithm, were applied to four typical network examples: NSFNET, ARPA2, SmallNet, and Bellcore, to validate and verify the $m$-cycle based FDL approaches. The performances of those $m$-cycle construction algorithms were compared in terms of the 
newly defined metrics. The results obtained from the example networks showed that the HST algorithm has better performance in terms of localization degree than HDFS and SPEM algorithms, while introduces the largest number of monitors and reserved wavelength for $m$-cycles. The SPEM algorithm produces the minimum number of $m$ cycles and thus has the best cost reduction and least wavelength overhead among the three algorithms. However, the localization degrees based on $m$-cycles generated by SPEM algorithm are the worst among three given algorithms. Instead, the HDFS algorithm accomplishes a trade-off between the localization degree and the wavelength overheads and costs.

In general, all the three $m$-cycle construction algorithms have good cost reduction over either monitor-per-link or monitor-per-path case. Furthermore, in a fair comparison of achieving complete localization, the HST algorithm has better cost reduction than HDFS and SPEM algorithms. Additionally, the wavelength overheads due to $m$-cycles are small or negligible depending on network capacity. Nevertheless, the wavelength overhead is still an important evaluation metric since it is not negligible in some other FDL schemes, e.g. FSM based FDL. The small wavelength overhead is one of the advantages of $m$-cycle based FDL approaches.

By extending the concept of degree- 2 chain, the necessary and sufficient condition for the complete fault localization based on $m$-cycles was proven to be that the topology of the AON contains neither degree- 2 chain nor extended degree- 2 chain. An immediate corollary was derived that every node of an AON is necessarily of degree-3 or above for achieving complete fault localization. It was also proven that if the complete fault localization is achievable in an AON, then the HST $m$-cycle construction algorithm could 
produce an $m$-cycle set to implement the complete fault localization.

To minimize the wavelength overhead and localization degree, and to consider the limitation of lightpath length in real AONs, the m-cycle construction was fully formulated into a variant version of constrained cycle-cover problem (vCCCP). A twophase branch-and-bound (B\&B) based algorithm was developed for solving the vCCCP. The results obtained by applying the two-phase vCCCP algorithm to both typical network examples from the real world and randomly generated network examples showed that this algorithm achieved a better performance than the previous algorithms in terms of both wavelength overhead and monitor cost (cycle number). Additionally, the algorithm is flexible in setting cost functions and is suitable for complex networks. The payment for the gains in performance is that this algorithm can only be applied to AON topologies satisfying the conditions for complete fault localization, as well its high time-complexity. An AON topology not satisfying such conditions has to be transformed before applying the algorithm. To make the research more statistically meaningful, the MFDL approach with the two-phase vCCCP algorithm was also applied to four randomly generated network examples. Analysis results on randomly generated examples lead to conclusions consistent to that in the examples from the real world, with regarding to the validality of the MFDL approach with the two-phase vCCCP algorithm, the performance of the algorithm such as the localization degree, the wavelength overhead, as well the cost reduction.

Finally, an implementation of the $m$-cycle based FDL scheme was discussed based on SNMP MIBs. The alarm delivery and faulty-source notification are carried by the common SNMP TRAP messages and SET messages. 
The MFDL approach has been developed and analyzed in this thesis for single-fault scenarios in AONs. However, such approaches can also be extended to multiple-fault scenarios (as shown in Section 3.3).

In conclusions, the work in this thesis has shown that the novel $m$-cycle based FDL approaches are effective and cost-efficient for today's ascending mesh AONs from the performance viewpoint. Such approaches are mostly applicable to AON metro or campus networks, where the cost of wavelengths is usually relatively lower than the optical monitors (especially for some expensive monitors like BERT and optical channel monitor). More importantly, the wavelengths already exist and usually the wavelength utilization is low (e.g., $10 \%$ to no more than $50 \%$ ). This implies that spare wavelengths are usually already established and available but extra monitor devices bring not only new investment of the devices themselves, but also the not-neglectable management expanse for them. Additionally, the operation, administration, maintenance (OAM), and monitoring equipments is also much higher than the cost of one or two wavelengths in an optical link. The only limitation of MFDL is that it is not sensible for some faults only affecting a single optical channel. Some in-site integrated monitoring modules might be deployed to detect this kind of faults as supplement of MFDL approaches.

Some potential future work that would add more depth and strength to this research topic is listed as the following:

- An open issue to this research left to the future work is to test a hypothesis that explains why HST has better performance over HDFS and then over SPEM.

- A related research topic is to incorporate the MFDL scheme with existing 
protection/restoration mechanisms in AONs. For example, the concept of p-cycle protection/restoration is inheritant close to the concept of m-cycle. Potentially, the $\mathrm{m}$-cycle can be also used as the p-cycle if some special constraits in cycle construction, e.g., the constraint the all links can be protected by at least one cycle.

- The detailed implementation design, such as $\mathrm{C} / \mathrm{C}++$ coding, for MFDL approaches might also be the focus of the future work. 


\section{References}

[1] T. E. Stern and K. Bala, Multiwavelength Optical Networks: A Layered Approach, Don Mills, ON: Addison-Wesley, 1999

[2] S. V. Kartalopoulos, Introduction to DWDM technology: data in a rainbow, New York: IEEE Press, 2000

[3] W. D. Grover, Mesh-based survivable networks: Options and strategies for optical, MPLS, SONET and ATM networking, Upper Saddle River, NJ: Prentice Hall, 2004

[4] S. Ramamurthy, L. Sahasrabuddhe, and B. Mukherjee, Survivable WDM mesh networks, IEEE/OSA Journal of Lightwave Technology, Vol. 21, Issue 4, pages $870-883,2003$

[5] O. Gerstel, R. Ramaswami, Optical layer survivability: a services perspective, IEEE Communications Magazine, Vol. 38, Issue 3, pages 104 - 113, Mar. 2000

[6] O. Gerstel and R. Ramaswami, Optical layer survivability-an implementation perspective, IEEE Journal on Selected Areas in Communications, Vol. 18, Issue 10, pages $1885-1899$, Oct. 2000

[7] C. Huang, V. Sharma, K. Owens, and S. Makam, Building reliable MPLS networks using a path protection mechanism, IEEE Communications Magazine, pages 2 - 8, Mar. 2002

[8] R.J. Patton, P.M. Frank, and R. N. Clark, Issues of Fault Diagnosis for Dynamic Systems, Springer, 2000

[9] P.J. Smith, D.W. Faulkner, and G.R. Hill, Evolution scenarios for optical 
telecommunication networks using multiwavelength transmission, Proceedings of the IEEE, Vol. 81, Issue 11, pages 1580-1587, Nov. 1993

[10] M. El-Sayed and J. Jaffe, A view of telecommunications network evolution, IEEE Communications Magazine, Vol. 40, Issue 12, pages 74-81, Dec. 2002

[11] IEEE Computer Society, Token ring access method and physical layer specifications, ANSI/IEEE Standard 802.5-1989, IEEE, 1989

[12] R. Ballart and Y.-C. Ching, SONET: Now it's the standard optical network, IEEE Communications Magazine, Vol. 27, Issue 3, pages 8-15, Mar. 1989

[13] R. Ballart and Y.-C. Ching, SONET: now it's the standard optical network, IEEE Communications Magazine, Vol. 40, Issue 5, pages 84-92, May 2002

[14] G.R. Hill, P.J. Chidgey, F. Kaufhold, T. Lynch, O. Sahlen, M. Gustavsson, M. Janson, B. Lagerstrom, G. Grasso, F. Meli, S. Johansson, J. Ingers, L. Fernandez, S. Rotolo, A. Antonielli, S. Tebaldini, E. Vezzoni, R. Caddedu, N. Caponio, F. Testa, A. Scavennec, M.J. O'Mahony, J. Zhou, A. Yu, W. Sohler, U. Rust, H. Herrmann, A transport network layer based on optical network elements, IEEE/OSA Journal of Lightwave Technology, Vol. 11, Issue 5, pages 667-679, May-June 1993

[15] C.A. Brackett, A.S. Acampora, J. Sweitzer, G. Tangonan, M.T. Smith, W. Lennon, K.-C. Wang, R.H. Hobbs, A scalable multiwavelength multihop optical network: a proposal for research on all-optical networks, IEEE/OSA Journal of Lightwave Technology, Vol. 11, Issue 5, pages 736-753, May-June 1993

[16] C. Mas and P. Thiran, A review on fault location methods and their applications in optical networks, Optical Network Magazine, Vol. 2, No. 4, 2001 
[17] R.H. Deng, A.A. Lazar, and W. Wang, A probabilistic approach to fault diagnosis in linear lightwave networks, IEEE Journal on Selected Areas in Communications, Vol. 11, Issue 9, pages 1438-1448, Dec. 1993

[18] M. Steinder and A. S. Sethi, Probabilistic fault localization in communication systems using belief networks, IEEE/ACM Transactions on Networking, Vol. 12, No. 5, pages 809-822, Oct. 2004

[19] N.S.V. Rao, On parallel algorithms for single-fault diagnosis in fault propagation graph systems, IEEE Transactions on Parallel and Distributed Systems, Vol. 7, No. 12, pages 1217-1223, Dec. 1996

[20] I. Katzela, G. Ellinas, W.S. Yoon, and T. E. Stern, Fault diagnosis in optical networks, Journal of High Speed Networks, Vol. 10, Issue 4, pages 269-291, 2001

[21] C. Mas and P. Thiran, An efficient algorithm for locating soft and hard failures in WDM networks, IEEE Journal on Selected Areas in Communications, Vol. 18, Issue 10, pages 1900-1911, Oct. 2000

[22] A.T. Bouloutas, G.W. Hart, and M. Schwartz, Fault identification using a finite state machine model with unreliable partially observed data sequences, IEEE Transactions on Communications, Vol. 41, No. 7, pages 1074-1083, July 1993

[23] C.-S. Li and R. Ramaswami, Automatic fault detection, localization, and recovery in transparent all-optical networks, IEEE Journal of Lightwave Technology, Vol. 15, No. 10, pages 1784-1793, Oct. 1997

[24] C. Rodriguez, S. Rementeria, J.I. Martin, A. Lafuente, J. Muguerza and J. Perez, A modular neural network approach to fault diagnosis, IEEE Transactions on Neural Networks, Vol. 7, No. 2, pages 326-340, Mar. 1996

$-149-$ 
[25] R. Weihmayer, G. Jakobson and M. Weissman, A domain oriented expert system for telecommunication network alarm correlation, in Proceedings of the 2nd IEEE Network Management and Control Workshop, Taqtown, N.Y. Sept. 21-23, 1993

[26] O. Gerstel and H. Raza, On the synergy between electrical and photonic switching, IEEE Communications Magazine, Vol. 41, Issue 4, pages. 98-104, 2003

[27] P. Bonenfant and A. Rodriguez-Moral, Optical data networking, IEEE Communications Magazine, No. 3, pages 63-70, 2000

[28] Y. Kobayashi, Y. Tada, S. Matsuoka, N. Hirayama, and K. Hagimoto, Supervisory systems for all-optical network transmission systems, in Proceedings of IEEE Globecom'96, pages 933-937, London, UK, Nov. 1996

[29] M. Goyal, K. K. Ramakrishnan, and W.-C. Feng, Achieving faster failure detection in OSPF networks, in Proceedings of IEEE ICC'03, Anchorage, Alaska, USA, May 11-15, 2003

[30] K. Kompella and G. Swallow, Detecting MPLS Data Plane Failures, IETF Internet-Draft, draft-ietf-mpls-lsp-ping-13.pdf, Jan. 2006

[31] H. Zeng, C. Huang, A. Vukovic, and M. Savoie, Achieving fast fault detection and localization in all-optical networks, in Proceedings of IEEE OFC/NEFOEC'05, Anaheim, CA, USA, Mar. 6-11, 2005

[32] C. Assi, Y. Ye, A. Shami, S. Dixit, and M. Ali, A hybrid distributed faultmanagement protocol for combating single-fiber failures in mesh-based DWDM optical networks, in Proceedings of IEEE Globecom'02, Taipei, Taiwan, Nov. 1721,2002

[33] Y. Ye, S. Dixit, M. Ali, On joint protection/restoration in IP-centric DWDM based $-150-$ 
optical transport networks, IEEE Communications Magazine, Vol. 38, Issue 6, pages $174-183$, June 2000

[34] Y. Qin, L. Mason and K. Jia, Study on a joint multiple layer restoration scheme for IP over WDM networks, IEEE Network Magazine, Vol. 17, Issue 2, pages 43 - 48, Mar.-Apr. 2003

[35] Y. Hamazumi, M. Koga, K. Kawai, H. Ichino, K. Sato, Optical path fault management in layered networks, in Proceedings of IEEE Globecom'98, Vol. 4, pages 2309-2314, Sydney, Australia, Nov. 8-12, 1998

[36] C. Mas, I. Tomkos, and O. K. Tonguz, Failure location algorithm for transparent optical networks, IEEE Journal on Selected Areas in Communications, Vol. 23, No. 8, pages 1508-1519, Aug. 2005

[37] S. Stanic, S. Subramaniam, H. Choi, G. Sahin, and H.-A. Choi, On monitoring transparent optical networks, in Proceedings of International Conference on Parallel Processing Workshops, pages 217-223, Vancouver, BC, Canada, Aug. 1821,2002

[38] T. Wu and A. K. Somani, Necessary and sufficient condition for k crosstalk attacks localization in all-optical networks, in Proceedings of IEEE Globecom'03, San Francisco, CA, USA, Dec. 1-5, 2003

[39] T. Wu, A. K. Somani, Attack monitoring and localization in all-optical networks, in Proceedings of SPIE, Vol. 4874, p. 235-248, OptiComm 2002: Optical Networking and Communications, Boston, MA, USA, July 2002

[40] R. Rabbat, Optical network failure recovery requirements, IETF Internet-Draft, draft-rabbat-optical-recovery-reqs-00.txt, June 2003 
[41] P. Czezowski, T. Soumiya, Optical network failure recovery requirements, IETF Internet-Draft, draft-czezowski-optical-recovery-reqs-01.txt, Mar. 2003

[42] R. Rabbat, Fault notification protocol for GMPLS-based recovery, IETF InternetDraft, draft-rabbat-fault-notification-protocol-03.txt, June 2003

[43] A. A. M. Saleh and J. M. Simmons, Architectural principles of optical regional and metropolitan access networks, IEEE/OSA Journal of Lightwave Technology, Vol. 17, Issue 12, pages 2431-2448, Dec. 1999

[44] A. E. Willner, M. C. Cardakli, O. H. Adamczyk, Y.-W. Song, and D. Gurkan, Key building blocks for all-optical networks, IEICE Transactions on Communications, Vol. E83-B, No. 10, pages 2166-2177, Oct. 2000

[45] H. Zeng, A. Vukovic, J. M. Savoie, and C. Huang, Optimisation of all-optical network testbed regarding NRZ and RZ modulation, in Proceedings of the 17th IEEE Canadian Conference on Electrical and Computer Engineering 2004 (IEEE CCECE'04), Vol. 1, pages 428-432, Niagara Falls, ON, Canada, May 2-5, 2004

[46] A. Vukovic, H. Zeng, M. Savoie, and H. Hua, Modeling and Optimization of Physical Layer Parameters for Development of Research Metro Networks, Photons, Vol. 3, No. 1, pages 19-21, Spring 2005

[47] H. Zeng, A. Vukovic, H. Hua, J. M. Savoie, and C. Huang, Optimisation of alloptical network testbed, in Proceedings of the 3rd IASTED International Conference on Wireless and Optical Communications (WOC'03), pages 75-79, Banff, Alberta, Canada, July 14-16, 2003

[48] D. R. Kuhn, Sources of failure in the public switched telephone network, IEEE Computer, Vol. 30, Issue 4, pages 31-36, April 1997 
[49] J. Zhang and B. Mukherjee, A review of fault management in WDM mesh networks: basic concepts and research challenges, IEEE Network, Vol. 18, Issue 2, pages 41-48, March/April 2004

[50] M. Médard, D. Marquis, and S. R. Chinn, Attack detection methods for all-optical networks, in Proceedings of IEEE Network and Distributed System Security Symposium, San Diego, California, Mar. 1998

[51] K.-U. Chu, C.-H. Lee, and S.-Y. Shin, Scalable optical-path supervisory scheme using pilot tones and channel equalizers, Electronics Letters, Vol. 36, Issue 9, 27 pages $817-818$, April 2000

[52] H. Zeng, A. Vukovic, C. Huang, H. Hua, and M. Savoie, Wavelength-routing fault detection in an AON testbed utilizing concatenated pilot tones, in Proceedings of SPIE Photonics North, Proc. SPIE 5579, Ottawa, Canada, Sept. 2004

[53] K. J. Park, C. J. Youn, J. H. Lee, and Y. C. Chung, Performance comparisons of chromatic dispersion-monitoring techniques using pilot tones, IEEE Photonics Technology Letters, Vol. 15, No. 6, pages 873 - 875, June 2003

[54] A. Kloch, S.L. Danielsen, B. Mikkelsen, K.E. Stubkjaer, M. Schilling, K. Wunstel, and W. Idler, Pilot tones in networks with nonlinear elements, IEEE Photonics Technology Letters, Vol. 10, Issue 3, pages 448 - 450, March 1998

[55] C. P. Larsen, P. O. Andersson, Signal quality monitoring in optical networks, Optical Networks Magazine, Vol. 1, No. 4, pages 17-23, Oct. 2000

[56] ITU-T Study group 15, Signal quality monitoring in optical networks, Aug. 1998

[57] S. V. Kartalopoulos, Fault Detectability in DWDM - Toward Higher Signal Quality \& System Reliability, Piscataway: IEEE Press, 2001

$$
-153-
$$


[58] K.-U. Chu, C.-H. Lee, and S.-Y. Shin, Scalable optical-path supervisory scheme using pilot tones and channel equalisers, Electronics Letters, Vol. 36, No. 9, pages $817-818,27$ April 2000

[59] M. To and P. Neusy, Unavailability analysis of long-haul networks, IEEE Journal on Selected Areas in Communications, Vol. 12, Issue 1, pages 100-109, Jan. 1994

[60] ITU-T G.798, Characteristics of optical transport network hierarchy equipment functional blocks, Jan. 2002

[61] W. D. Wallis, A Beginner's guide to graph theory, Boston: Birkhaeuser, 2000

[62] F. Jarger, A survey of the cycle double cover conjecture, in: Cycles in Graphs, Edited by B. R. Alspach and C. D. Godsil, Annals of discrete mathematics 27, New York: Elsevier Science, 1985

[63] C.-Q. Zhang, Integer flows and cycle covers of graphs, New York: Marcel Dekker, 1997

[64] D. B. West, Introduction to graph theory, Upper Saddle River, NJ: Prentice Hall, 2001

[65] F. Jarger, and T. Awart, "Conjecture 1," in: Combinatorics 79, Edited by M. Deza and I. G. Rosenberg, Annals of discrete mathematics 9, New York: Elsevier Science, 1980

[66] I. M. Isaacs, Algebra: a graduate course, Pacific Grove, Calif.: Brooks/Cole Pub. Co., 1994

[67] R. Elliott, Dark fibre pricing analysis Europe 1998-2002, [Online document], Nov. 2002, available at http://www.band-x.com/information/Dark_Fibre_Reportprices_98-02webversion.pdf 
[68] A. K. Bjerring and B. St. Arnaud, Can Canada be a world leader in the next Internet Revolution? [Online document], March 2002, available at http://www.canarie.ca/advnet/fibre.html

[69] A. Itai and M. Rodeh, Covering a graph by circuits, Automata, languages and programming, Lecture Notes in Computer Science 62, pages 289-299, Berlin: Springer-Verlag, 1978

[70] G. Fan, Covering graphs by cycles, SIAM journal on Discrete Mathematics, Vol. 5, No. 4, pages 491-496, Nov. 1992

[71] L. M. Gardner, M. Heydari, J. Shah, I. H. Sudborough, I. G. Tollis and C. Xia, Techniques for finding ring covers in survivable networks, in Proceedings of IEEE Globecom'94, pages 1862-1866, San Francisco, USA, 28 Nov. - 2 Dec. 1994

[72] H. Zeng, C. Huang, and A. Vukovic, Monitoring cycles for fault detection in meshed all-optical networks, in Proceedings of 2004 International Conference on Parallel Processing Workshops (IEEE ICPP'04), Pages 434-439, Montreal, Quebec, Canada, Aug. 15-18, 2004

[73] R.J. Wilson and J.J. Watkins, Graphs: an introductory approach, New York: Wiley, 1990

[74] H. Zeng, C. Huang, A. Vukovic and M. Savoie, Fault detection and path performance monitoring in meshed all-optical networks, in Proceedings of IEEE Global Telecommunications Conference 2004 (IEEE GLOBECOM'04), Dallas, TX, USA, 29 Nov.-3 Dec. 2004

[75] H. Walther, Chapter 1: Flows and tensions on networks, Ten applications of graph theory, Boston: D. Reidel Pub. Co., 1984 
[76] H. Zeng, C. Huang, and A. Vukovic, Spanning-tree based monitoring-cycle construction for fault detection and localization in meshed all-optical networks, to appear in Proceedings of IEEE international Conference on Communications (IEEE ICC'05), Seoul, Korea, May 16-20, 2005

[77] H.A. Eiselt and C.-L. Sandblom, Integer programming and network models, Berlin, New York: Springer, 2000

[78] E. K.P. Chong and S. H. Zak, An introduction to optimization, New York: Wiley, 2001

[79] G. P. McCormick, Nonlinear programming: theory, algorithms, and applications, New York: Wiley, 1983

[80] I. Katzela and M. Schwartz, Schemes for fault identification in communication networks, IEEE/ACM Transactions on Networking, Vol. 3, No. 6, Dec. 1995

[81] C. Wang and M. Schwartz, Identification of faulty links in dynamic-routed networks, IEEE Journal on Selected Areas in Communications, Vol. 11, No. 9, Dec. 1993

[82] T. Wu and A. K. Somani, Cross-talk attack monitoring and localization in alloptical networks, IEEE/ACM Transactions on Networking, Vol. 13, No. 6, Dec. 2005

[83] C. S. Hood and C. Ji, Proactive network fault detection, IEEE Transactions on Reliability, Vol. 46, No. 3, Sept. 1997

[84] H. Cross, Analysis of flows in networks of conducts of conductors, Bulletin No. 285, University of Illinois Engineering Experimental Station, Urbana, Illinois, 1936 
[85] H. Zeng, C. Huang, and A. Vukovic, A Novel Fault Detection and Localization Scheme for Mesh All-Optical Networks Based on Monitoring-Cycles, Photonic Network Communications, Vol. 11, No. 3, May 2006

[86] C. Thomassen, On the complexity of finding a minimum cycle cover of a graph, SIAM Journal on Computing, Vol. 26, pages 675-677, 1997

[87] M. Guan, Graphic programming using odd and even points, Chinese Mathematics, Vol. 1, pages 273-277, 1962

[88] J. Edmonds and E. L. Johnson, Matching, Euler tours and Chinese postman problem, Mathematical Programming, Vol. 5, pages 88-124, 1973

[89] A. Itai, R. J. Lipton, C. H. Papadimitrious, and M. Rodeh, Covering graphs by simple circuits, SIAM Journal on Computing, Vol. 10, pages 746-750, 1981

[90] J. C. Bermond, B. Jackson, and F. Jaeger, Shortest covering of graphs with cycles, Journal of Combinatorial Theory B, Vol. 35, pages 297-308, 1983

[91] N. Alon and M. Tarsi, Covering multigraphs by simple circuits, SLAM Journal on Algebraic and Discrete Methods, Vol. 6, pages 345-350, 1985

[92] P. Fraisse, Cycle covering in bridgeless graphs, Journal of Combinatorial Theory $B$, Vol. 39, pages 146-152, 1985

[93] G. Fan, Extension of flow theorems, Journal of Combinatorial Theory B, Vol. 69, pages 110-114, 1997

[94] G. Fan, Minimum cycle covers of graphs, Journal of Graph Theory, Vol. 25, pages 229-242, 1997

[95] D. M. Chickering, D. Geiger, and D. Heckerman, On finding a cycle basis with a shortest maximal cycle, Information Processing Letters, Vol. 54, pages 55-58, 
1995

[96] M. Labbe, G. Laporte, and P. Soriano, Covering a graph with cycles, Computers and Operations Research, Vol. 25, pages 499-504, 1998

[97] N. Immorlica, M. Mahdian, and V. S. Mirrokni, Cycle cover with short cycles, 22nd Annual Symposium on Theoretical Aspects of Computer Science, Stuttgart, Germany, February 24-26, 2005, (STACS 2005), Lecture Notes in Computer Science, Vol. 3404, pp. 641-653, 2005

[98] D. S. Hochbaum and E. V. Olinick, The bounded cycle-cover problem, INFORMS Journal on Computing, Vol. 13, pages 104-119, 2001

[99] M. R. Garey and D. S. Johnson, Computers and Intractability: $A$ Guide to the Theory of NP-Completeness, San Francisco, CA: W. H. Freeman, 1979

[100] G. Pesant and P. Soriano, An optimal strategy for the constrained cycle cover problem, Annals of Mathematics and Artificial Intelligence, Vol. 34, pages 313325,2002

[101] W. A. Shay, Understanding data communications and networks, Belmont, CA: Brooks/Cole - Thomas Learning, 2004

[102] W. L. Winston, Introduction to mathematical programming applications and algorithms, Belmont, CA: Duxbury, 1995

[103] Y. Wen, V. W. S. Chan, and L. Zheng, Efficient fault-diagnosis algorithms for alloptical WDM networks with probabilistic link failures, IEEE/OSA Journal of Lightwave Technology, Vol. 23, No. 10, pp. 3358-3371, Oct. 2005

[104] J. Moy, OSPF Version 2, IETF Request for Comments 2328, April 1998

[105] D. Oran, "OSI IS-IS intra-domain routing protocol, IETF Request for Comments 
1142, Feb. 1990

[106] W. V. Wollman and Y. Barsoum, Overview of Open Shortest Path First, version 2 routing in the tactical environment, in Proceedings of IEEE Military Communications Conference (MILCOM' 95), pp. 925 - 930, San Diego, CA, USA, Nov. 1995

[107] Anonymous, Synchronous optical network (SONET), the International Engineering Consortium (IEC), http://www.iec.org/online/tutorials/sonet/index.html

[108] K. Kompella, P. Pan, D. Cooper, G. Swallow, S. Wadhwa, and R. Bonica, Detecting MPLS Data Plane Failures, IETF Internet-Draft, draft-ietf-mpls-lspping-00.pdf, Mar. 2002

[109] K. Kompella and G. Swallow, Detecting MPLS Data Plane Failures, IETF RFC 4379, Feb. 2006

[110] A. G. Hailemariam, G. Ellinas, and T. Stern, Localized failure restoration in mesh optical networks, in Proceedings of Optical Fiber Communication Conference, 2004 (OFC 2004), Vol. 1, Feb. 2004

[111] H. Zeng, A. Vukovic, C. Huang, H. Hua, and M. Savoie, A novel end-to-end fault detection and localization protocol for wavelength-routed WDM networks, in Proceedings of SPIE Photonics North 2005, Toronto, Canada, Sept. 2005

[112] A. V. Sichani and H. T. Mouftah, A novel broadcasting fault detection protocol in all-optical WDM networks, in Proceedings of Queens Biennial Symposium on Communications ( $Q B S C^{\prime} 04$ ), pages 222-224, May 2004

[113] A. V. Sichani and H. T. Mouftah, Rolling back signaling protocol - A novel fault localization WDM mesh networks, CIC China Communications Magazine, Vol. 1, 
No. 1, pages 101-105, Dec. 2004

[114] J. Y. Wei, Advances in the management and control of optical Internet, IEEE Journal on Selected Areas in Communications, Vol. 20, No. 4, pages 768-785, May 2002

[115] B. Rajagopalan, J. Luciani, and D. Awduche, IP over optical networks: A framework, IETF RFC 3717, Mar. 2004

[116] G. Li, J. Yates, D. Wang, and C. Kalmanek, Control plane design for reliable optical networks, IEEE Communications Magazine, Vol. 40, No.2, pages 90-96, Feb. 2002

[117] J. Wu, M. Savoie, and H. Mouftah, Recovery from control plane failures in the LDP signaling protocol, Optical Switching and Networking, Vol. 2, No. 3, pages 148-162, Nov., 2005

[118] J. Wu, J. M. Savoie, D. Y. Montuno, and H. T. Mouftah, Recovery from control plane failures in the CR-LDP signaling protocol, in Proceedings of IEEE International Conference on Communications, Vol. 2, pages 1309-1313, 2003

[119] J. Wu, D. Y. Montuno, H. T. Mouftah, G. Wang, and A. C. Dasylva, Recovery from control plane failures in GMPLS-controlled optical networks, International Journal of Communication Systems, Vol. 15, No. 7, pages 573-592, Sept., 2002

[120] Microsoft TechNet, Architecture blueprints: Management architecture blueprint, [Online document: accessed on Mar 21, 2006], Mar. 31, 2005, available at http://microsoft.com/technet/itsolutions/wssra/raguide/ArchitectureBluePrints

[121] J. Case, M. Fedor, M. Schoffstall, and J. Davin, A Simple Network Management Protocol, IETF RFC 1067, Aug. 1988 
[122] J. Case, M. Fedor, M. Schoffstall, and J. Davin, A Simple Network Management Protocol (SNMP), IETF RFC 1157, May 1990

[123] M. Rose and K. McCloghrie, Structure and Identification of Management Information for TCP/IP-based Internets, IETF RFC 1155, May 1990

[124] J. Case, K. McCloghrie, M. Rose, and S. Waldbusser, Structure of Management Information for Version 2 of the Simple Network Management Protocol (SNMPv2), IETF RFC 1902, Jan. 1996

[125] ITU-T, Abstract Syntax Notation One (ASN.1): Specification of Basic Notation, ITU-T Rec. X.680 (2002) | ISO/IEC 8824-1:2002

[126] ITU-T, Abstract Syntax Notation One (ASN.1): Information Object Specification, ITU-T Rec. X.681 (2002) | ISO/IEC 8824-2:2002

[127] ITU-T, Abstract Syntax Notation One (ASN.1): Constraint Specification, ITU-T Rec. $X .682$ (2002) | ISO/IEC 8824-3:2002

[128] ITU-T, Abstract Syntax Notation One (ASN.1): Parameterization of ASN.1 Specifications, ITU-T Rec. X.683 (2002) | ISO/IEC 8824-4:2002

[129] K. McCloghrie and M. Rose, Management Information Base for Network Management of TCP/IP-based internets: MIB-II, IETF RFC 1213, Mar. 1991

[130] J. Zheng and H. T. Mouftah, Destination-initiating path restoration protocol for wavelength-routed WDM networks, IEE Proceedings on Communications, Vol. 149, No. 1, pages 18-22, Feb. 2002

[131] T. H. Cormen, C. E. Leiserson, R. L. Rivest, and C. Stein, Introduction to algorithms, 2nd Edition, MIT Press and McGraw-Hill, 2001

[132] A. Medina, A. Lakhina, I. Matta, and J. Byers, BRITE [online resource]

$$
-161-
$$


http://www.cs.bu.edu/brite/

[133] S. G. Wilson, Digital Modulation and Coding, Englewood Cliffs, N.J.: PrenticeHall, 1996

[134] C. Guéret, N. Jussien, and C. Prins, Using intelligent backtracking to improve branch and bound methods: an application to Open-Shop problems, European Journal of Operational Research, Vol. 127, No. 2, pp. 344-354, 2000 


\section{Appendix A}

\section{Network examples and MFLA Results}

\section{with HDFS/SPEM Algorithms}

This appendix lists the figures of typical network examples in Chapter 4.4:
NSFNET, ARPA2, SmallNet, and Bellcore [3]. The $m$-cycles for these network examples obtained by two different $m$-cycle construction algorithms: HDFS and SPEM, are given in the corresponding figures. All nodes in each network example are numbered from zero and cycles, paths are represented by sequences of node numbers that are in them.

\section{A.1 $M$-cycles obtained by HDFS algorithm}




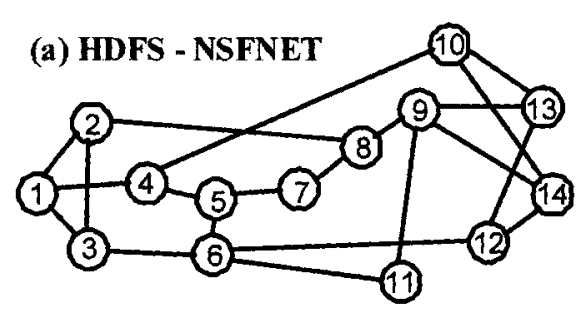

m-cycles obtained by HDFS:

cycle 1: $\quad 10-14-12-13-10$

cycle 2: 1-2-3-1

cycle 3: $\quad 5-7-8-9-11-6-5$

cycle 4: 4-1-2-8-7-5-4

cycle 5: $9-14-12-6-3-2-8-9$

cycle 6: $\quad 4-10-13-9-8-2-1-4$

(c) HDFS - SmallNet

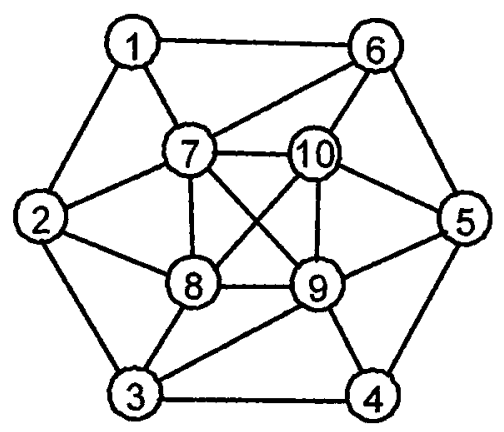

m-cycles obtained by HDFS:

cycle 1: $\quad 10-9-5-6-10$

cycle 2: $1-2-3-4-5-10-7-1$

cycle 3: $8-10-9-8$

cycle 4: $6-1-2-7-6$

cycle 5: $9-4-5-10-7-9$

cycle 6: $9-4-3-8-9$

cycle 7: $7-8-2-1-7$

cycle 8: $\quad 3-9-4-3$ (b) HDFS - ARPA2

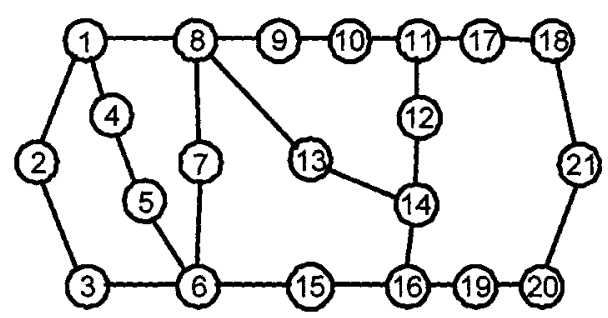

m-cycles obtained by HDFS:

cycle 1:16-19-20-21-18-17-11-12-14-

16

cycle $2:$ 2-1-4-5-6-3-2

cycle 3: 8-9-10-11-12-14-16-15-6-7-8

cycle 4: 1-8-13-14-16-15-6-3-2-1 (d) HDFS - Bellcore

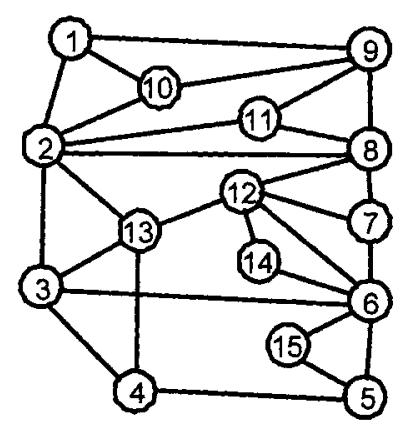

m-cycles obtained by HDFS:

Cycle 1: 12-14-6-15-5-4-13-12

Cycle 2: 2-3-4-5-6-7-8-2

Cycle 3: 11-8-12-7-6-3-13-2-11

Cycle 4: $1-2-10-9-1$

Cycle 5: 9-11-8-9

Cycle 6: 1-10-2-3-4-5-6-12-7-8-9-1

Figure A.1 $M$-cycles obtained by HDFS for network examples

(a) $m$-cycles obtained by HDFS in NSFNET; (b) $m$-cycles obtained by HDFS in ARPA2;

(c) $m$-cycles obtained by HDFS in SmallNet; (d) $m$-cycles obtained by HDFS in Bellcore. 


\section{A.2 $M$-cycles obtained by SPEM algorithm}

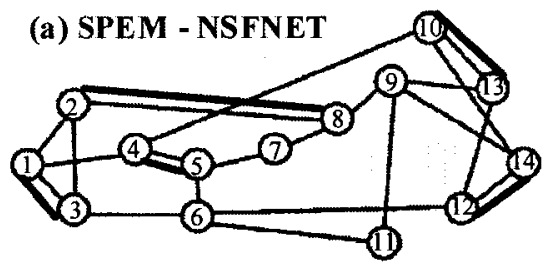

Eule ra in circ uit

1-4-5-4-10-14-12-13-10-13-9-14-12-6-

$11-9-8-7-5-6-3-2-8-2-1-3-1$

$\boldsymbol{m}$-cycle obtained by SPEM:

cycle 1: 1-4-5-4-10-13-9-8-2-1-3-1

cycle 2: $10-14-12-13-10$

cycle $3:$ 9-14-12-6-11-9

cycle 4: 8-7-5-6-3-2-8

(c) SPEM - S mallNet

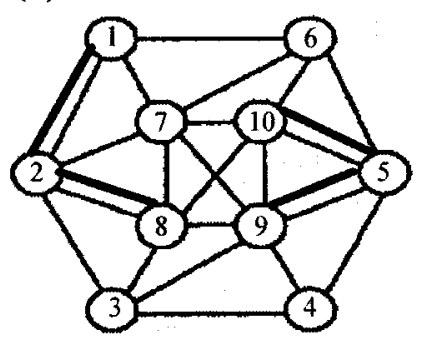

Eule ra in circuit

1-7-10-9-8-7-9-5-10-6-7-2-3-9-4$5-6-1-2-8-10-5-4-3-8-2-1$

$\boldsymbol{m}$-cycles obtained by SPEM:

cycle 1: 7-10-9-8-7

cycle $2: 9-5-10-6-7-2-3-9$

cycle 3: 5-6-1-2-8-10-5

cycle 4: 1-7-9-4-5-4-3-8-2-1 (b) SPEM - ARPA2

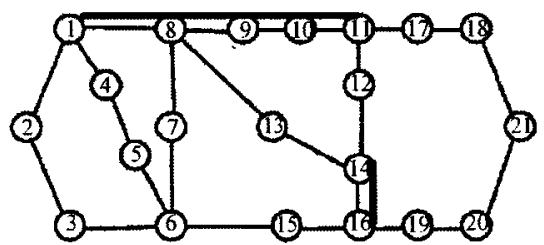

Eule ra in circuit

1-8-13-14-16-19-20-21-18-17-11-12-14-1615-6-7-8-9-10-11-10-9-8-1-4-5-6-3-2-1

$\boldsymbol{m}$-cycles obtained by SPEM:

cycle 1: 1-8-13-14-16-15-6-7-8-1

cycle 2: 14-16-19-20-21-18-17-11-12-14

cycle 3: 8-9-10-11-10-9-8

cycle 4: $1-4-5-6-3-2-1$

(d) SPEM - Bellco re

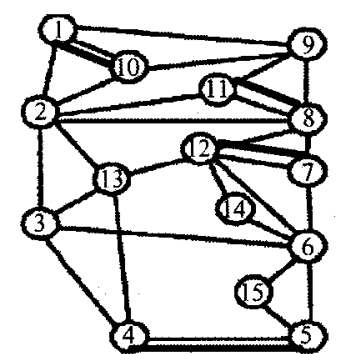

Eule ra in circuit

$1-10-9-11-8-12-14-6-15-5-6-12-13-4-5-4-$ 3-13-2-11-8-9-1-10-2-3-6-7-12-7-8-2-1

\section{m-cycles obtained by SPEM:}

cycle 1:1-10-9-11-8-2-1

cycle $2: 8-12-13-2-3-6-7-12-7-8$

cycle $3: 12-14-6-15-5-6-12$

cycle $4: 13-4-5-4-3-13$

cycle 5:2-11-8-9-1-10-2

Legend: Shortest-path Eulerian matching

Figure A.2 $M$-cycles obtained by SPEM for network examples

(a) $m$-cycles obtained by SPEM in NSFNET; (b) $m$-cycles obtained by SPEM in ARPA2;

(c) $m$-cycles obtained by SPEM in SmallNet; (d) $m$-cycles obtained by SPEM in Bellcore. 


\section{A.3 MFLA results with $M$-cycles obtained by HDFS}

\section{algorithm}

Table A.1 Fault localization results with $m$-cycles obtained by HDFS in NSFNET

\begin{tabular}{|c|c|c|c|c|c|c|}
\hline \multicolumn{6}{|c|}{ Alarm code } & \multirow{2}{*}{ Faulty link candidates } \\
\hline $\mathrm{cl}$ & $\mathrm{c} 2$ & c3 & $\mathrm{c4}$ & c5 & c6 & \\
\hline 0 & 0 & 0 & 0 & 0 & 0 & null \\
\hline 0 & 0 & 0 & 0 & 0 & 1 & $(4,10),(9,13)$ \\
\hline 0 & 0 & 0 & 0 & 1 & 0 & $(3,6),(6,12),(9,14)$ \\
\hline 0 & 0 & 0 & 1 & 0 & 0 & $(4,5)$ \\
\hline 0 & 0 & 0 & 1 & 0 & 1 & $(1,4)$ \\
\hline 0 & 0 & 0 & 1 & 1 & 1 & $(2,8)$ \\
\hline 0 & 0 & 1 & 0 & 0 & 0 & $(5,6),(6,11),(9,11)$ \\
\hline 0 & 0 & 1 & 0 & 1 & 1 & $(8,9)$ \\
\hline 0 & 0 & 1 & 1 & 0 & 0 & $(5,7),(7,8)$ \\
\hline 0 & 1 & 0 & 0 & 0 & 0 & $(1,3)$ \\
\hline 0 & 1 & 0 & 0 & 1 & 0 & $(2,3)$ \\
\hline 0 & 1 & 0 & 1 & 0 & 1 & $(1,2)$ \\
\hline 1 & 0 & 0 & 0 & 0 & 0 & $(10,13), \quad(10,14)$ \\
\hline 1 & 0 & 0 & 0 & 0 & 1 & $(12,13)$ \\
\hline 1 & 0 & 0 & 0 & 1 & 0 & $(12,14)$ \\
\hline & & & & & & N/A \\
\hline
\end{tabular}


Table A.2 Fault localization results with $m$-cycles obtained by HDFS in ARPA2

\begin{tabular}{cccr|l}
\hline \multicolumn{3}{c|}{ Alarm code } & \multicolumn{1}{|}{ Faulty link candidates } \\
\cline { 1 - 2 } $\mathrm{c4}$ & $\mathrm{c} 3$ & $\mathrm{c} 2$ & $\mathrm{c} 1$ & \multicolumn{1}{|c}{ null } \\
\hline 0 & 0 & 0 & 0 & \multicolumn{1}{|c}{} \\
0 & 0 & 0 & 1 & $(11,17),(16,19),(17,18),(18,21),(19,20),(20,21)$ \\
0 & 0 & 1 & 0 & $(1,4),(4,5),(5,6)$ \\
0 & 1 & 0 & 0 & $(6,7),(7,8),(8,9),(9,10),(10,11)$ \\
0 & 1 & 0 & 1 & $(11,12),(12,14)$ \\
1 & 0 & 0 & 0 & $(1,8),(8,13),(13,14)$ \\
1 & 0 & 1 & 0 & $(1,2),(2,3)) 3,6)$, \\
1 & 1 & 0 & 0 & $(6,15),(15,16)$ \\
1 & 1 & 0 & 1 & $(14,16)$ \\
& Others & & N/A \\
\hline
\end{tabular}

$-167-$ 
Table A.3 Fault localization results with $m$-cycles obtained by HDFS in SmallNet

\begin{tabular}{|c|c|c|c|c|c|c|c|c|}
\hline \multicolumn{8}{|c|}{ Alarm code } & \multirow{2}{*}{ Faulty link candidates } \\
\hline c8 & c7 & c6 & c5 & $\mathrm{c} 4$ & c3 & $\mathrm{c} 2$ & $\mathrm{cl}$ & \\
\hline 0 & 0 & 0 & 0 & 0 & 0 & 0 & 0 & null \\
\hline 0 & 0 & 0 & 0 & 0 & 0 & 0 & 1 & $(5,6),(5,9),(6,10)$ \\
\hline 0 & 0 & 0 & 0 & 0 & 0 & 1 & 0 & $(2,3)$ \\
\hline 0 & 0 & 0 & 0 & 0 & 1 & 0 & 0 & $(8,10)$ \\
\hline 0 & 0 & 0 & 0 & 0 & 1 & 0 & 1 & $(9,10)$ \\
\hline 0 & 0 & 0 & 0 & 1 & 0 & 0 & 0 & $(1,6),(2,7),(6,7)$ \\
\hline 0 & 0 & 0 & 1 & 0 & 0 & 0 & 0 & $(7,9)$ \\
\hline 0 & 0 & 0 & 1 & 0 & 0 & 1 & 0 & $(4,5),(5,10),(7,10)$ \\
\hline 0 & 0 & 1 & 0 & 0 & 0 & 0 & 0 & $(3,8)$ \\
\hline 0 & 0 & 1 & 0 & 0 & 1 & 0 & 0 & $(8,9)$ \\
\hline 0 & 1 & 0 & 0 & 0 & 0 & 0 & 0 & $(2,8),(7,8)$ \\
\hline 0 & 1 & 0 & 0 & 0 & 0 & 1 & 0 & $(1,7)$ \\
\hline 0 & 1 & 0 & 0 & 1 & 0 & 1 & 0 & $(1,2)$ \\
\hline 1 & 0 & 0 & 0 & 0 & 0 & 0 & 0 & $(3,9)$ \\
\hline 1 & 0 & 1 & 0 & 0 & 0 & 1 & 0 & $(3,4)$ \\
\hline 1 & 0 & 1 & 1 & 0 & 0 & 0 & 0 & $(4,9)$ \\
\hline \multicolumn{8}{|c|}{ Others } & N/A \\
\hline
\end{tabular}


Table A.4 Fault localization results with $m$-cycles obtained by HDFS in Bellcore

\begin{tabular}{|c|c|c|c|c|c|c|}
\hline \multicolumn{6}{|c|}{ Alarm code } & \multirow{2}{*}{ Faulty link candidates } \\
\hline c6 & c5 & $\mathrm{c4}$ & c3 & $\mathrm{c} 2$ & $\mathrm{cl}$ & \\
\hline 0 & 0 & 0 & 0 & 0 & 0 & null \\
\hline 0 & 0 & 0 & 0 & 0 & 1 & $(4,13),(5,15),(6,14),(6,15),(12,13),(12,14)$ \\
\hline 0 & 0 & 0 & 0 & 1 & 0 & $(2,8)$ \\
\hline 0 & 0 & 0 & 1 & 0 & 0 & $(2,11),(2,13),(3,6),(3,13),(8,12)$ \\
\hline 0 & 0 & 0 & 1 & 1 & 0 & $(6,7)$ \\
\hline 0 & 0 & 1 & 0 & 0 & 0 & $(1,2),(9,10)$ \\
\hline 0 & 1 & 0 & 0 & 0 & 0 & $(9,11)$ \\
\hline 0 & 1 & 0 & 1 & 0 & 0 & $(8,11)$ \\
\hline 1 & 0 & 0 & 0 & 0 & 0 & $(1,10),(6,12)$ \\
\hline 1 & 0 & 0 & 0 & 1 & 0 & $(2,3),(3,4),(5,6),(7,8)$ \\
\hline 1 & 0 & 0 & 0 & 1 & 1 & $(4,5)$ \\
\hline 1 & 0 & 0 & 1 & 0 & 0 & $(7,12)$ \\
\hline 1 & 0 & 1 & 0 & 0 & 0 & $(1,9),(2,10)$ \\
\hline 1 & 1 & 0 & 0 & 0 & 0 & $(8,9)$ \\
\hline \multicolumn{6}{|c|}{ Others } & N/A \\
\hline
\end{tabular}




\section{A.4 MFLA results with $M$-cycles obtained by SPEM algorithm}

Table A.5 Fault localization results with $m$-cycles obtained by SPEM in NSFNET

\begin{tabular}{|c|c|c|c|c|}
\hline \multicolumn{4}{|c|}{ Alarm code } & \multirow{2}{*}{ Faulty link candidates } \\
\hline $\mathrm{c4}$ & c3 & $\mathrm{c} 2$ & $\mathrm{cl}$ & \\
\hline 0 & 0 & 0 & 0 & Null \\
\hline 0 & 0 & 0 & 1 & $(1,2),(1,3),(1,4),(4,5),(4,10),(8,9),(9,13)$ \\
\hline 0 & 0 & 1 & 0 & $(10,14),(12,13)$ \\
\hline 0 & 0 & 1 & 1 & $(10,13)$ \\
\hline 0 & 1 & 0 & 0 & $(6,11),(6,12),(9,11),(9,14)$ \\
\hline 0 & 1 & 1 & 0 & $(12,14)$ \\
\hline 1 & 0 & 0 & 0 & $(2,3),(3,6),(5,6),(5,7),(7,8)$ \\
\hline 1 & 0 & 0 & 1 & $(2,8)$ \\
\hline \multicolumn{4}{|c|}{ Others } & N/A \\
\hline
\end{tabular}


Table A.6 Fault localization results with $m$-cycles obtained by SPEM in ARPA2

\begin{tabular}{|c|c|c|c|c|}
\hline \multicolumn{4}{|c|}{ Alarm codes } & \multirow{2}{*}{ Faulty link candidates } \\
\hline $\mathrm{c4}$ & c3 & $\mathrm{c} 2$ & c1 & \\
\hline 0 & 0 & 0 & 0 & Null \\
\hline 0 & 0 & 0 & 1 & $(1,8),(6,7),(6,15),(7,8),(8,13),(13,14),(15,16)$ \\
\hline 0 & 0 & 1 & 0 & $\begin{array}{l}(11,12),(11,17),(12,14),(16,19),(17,18),(18,21), \\
(19,20),(20,21)\end{array}$ \\
\hline 0 & 0 & 1 & 1 & $(14,16)$ \\
\hline 0 & 1 & 0 & 0 & $(8,9),(9,10),(10,11)$ \\
\hline 1 & 0 & 0 & 0 & $(1,2),(1,4),(2,3),(3,6),(4,5),(5,6)$ \\
\hline \multicolumn{4}{|c|}{ Others } & N/A \\
\hline
\end{tabular}

Table A.7 Fault localization results with $m$-cycles obtained by SPEM in SmallNet

\begin{tabular}{cccc|c}
\hline \multicolumn{3}{c|}{ Alarm code } & \multicolumn{1}{|c}{ Faulty link candidates } \\
\hline $\mathrm{c} 4$ & $\mathrm{c} 3$ & $\mathrm{c} 2$ & $\mathrm{c} 1$ & \multicolumn{1}{|c}{ Null } \\
\hline 0 & 0 & 0 & 0 & \multicolumn{1}{|c}{} \\
0 & 0 & 0 & 1 & $(7,8),(7,10),(8,9),(9,10)$ \\
0 & 0 & 1 & 0 & $(2,3),(2,7),(3,9),(5,9),(6,7),(6,10)$ \\
0 & 1 & 0 & 0 & $(1,6),(5,6),(8,10)$ \\
0 & 1 & 1 & 0 & $(5,10)$ \\
1 & 0 & 0 & 0 & $(1,7),(3,4),(3,8),(4,5),(4,9),(7,9)$ \\
1 & 1 & 0 & 0 & $(1,2),(2,8)$ \\
& Others & & N/A \\
\hline
\end{tabular}


Table A.8 Fault localization results with $m$-cycles obtained by SPEM in Bellcore

\begin{tabular}{|c|c|c|c|c|c|}
\hline \multicolumn{5}{|c|}{ Alarm code } & \multirow{2}{*}{ Faulty link candidates } \\
\hline c5 & $\mathrm{c} 4$ & c3 & c2 & $\mathrm{cl}$ & \\
\hline 0 & 0 & 0 & 0 & 0 & nu11 \\
\hline 0 & 0 & 0 & 0 & 1 & $(1,2),(2,8),(9,10),(9,11)$ \\
\hline 0 & 0 & 0 & 1 & 0 & $\begin{array}{l}(2,3),(2,13),(3,6),(6,7),(7,8),(7,12),(8,12), \\
(12,13)\end{array}$ \\
\hline 0 & 0 & 1 & 0 & 0 & $(5,6),(5,15),(6,12),(6,14),(6,15),(12,14)$ \\
\hline 0 & 1 & 0 & 0 & 0 & $(3,4),(3,13),(4,5),(4,13)$ \\
\hline 1 & 0 & 0 & 0 & 0 & $(1,9),(2,10),(2,11),(8,9)$ \\
\hline 1 & 0 & 0 & 0 & 1 & $(1,10),(8,11)$ \\
\hline \multicolumn{5}{|c|}{ Others } & N/A \\
\hline
\end{tabular}




\section{Appendix B}

\section{Network examples and MFLA Results}

\section{with HST Algorithms}

This appendix lists the fault localization results obtained by the MFLA upon
network examples in Chapter 4 (NSFNET, ARPA2, SmallNet, and Bellcore), together with $m$-cycles built by HST algorithms.

\section{B.1 $M$-cycles obtained by HST algorithm}




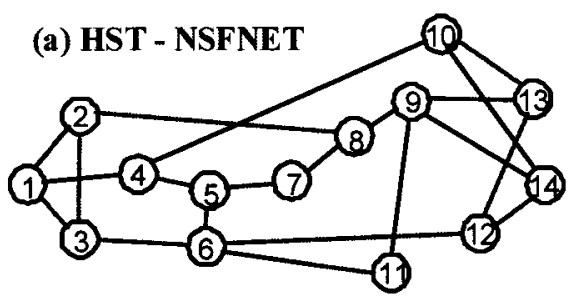

m-cycles chord

cycle 1: $\quad$ 1-2-3-1

cycle 2: 1-4-5-6-3-1

cycle 3: 4-10-13-12-6-5-4

cycle 4: $\quad$ 7-8-2-3-6-5-7

cycle 5: $\quad$ 8-9-13-12-6-3-2-8

cycle 6: 9-11-6-12-13-9

cycle 7: $\quad$ 9-14-12-13-9

cycle 8: $\quad \mathbf{1 0 - 1 4 - 1 2 - 1 3 - 1 0}$

(c) HST - SmallNet

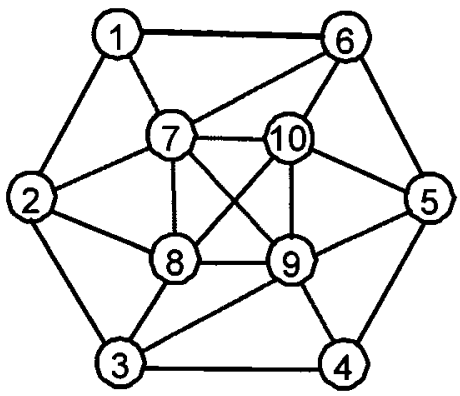

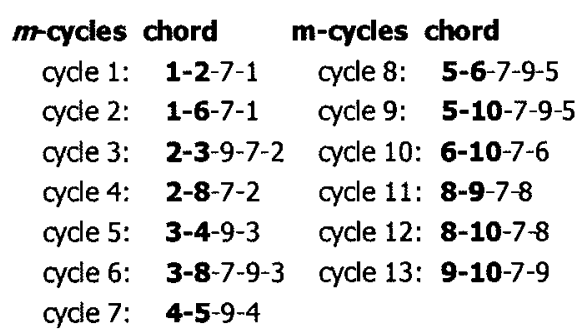

(b) HST - ARPA2

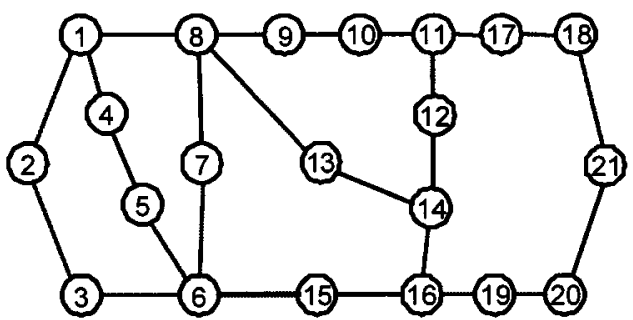

m-cycles chord

cycle 1: 4-5-6-3-2-1-4

cycle 2: $\quad$ 7-8-1-2-3-6-7

cycle 3: 13-14-12-11-10-9-8-13

cycle 4: 15-16-14-12-11-10-9-8-1-

2-3-6-15

cycle 5: $\quad 20-21-18-17-11-12-14-16-$

19-20

(d) HST - Bellcore

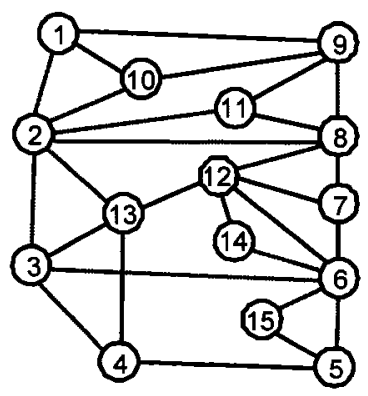

m-cycles chord m-cycles chord

cycle 1: 1-9-8-2-1 cycle 8: 5-15-6-5

cycle 2: 1-10-2-1 cycle 9: 7-12-8-7

cycle 3: 3-13-2-3 cycle 10: 8-11-2-8

cycle 4: 4-5-6-3-4 cycle 11: 9-10-2-8-9

cycle 5: 4-13-2-3-4 cycle 12: 9-11-2-8-9

cycle 6: $6-7-8-2-3-6$ cycle $13: 12-13-2-8-12$

cycle 7: $6-12-8-2-3-6$ cycle $14: 12-14-6-3-2-8-12$

\section{Legend: - Spanning-tree}

Figure B.1 $M$-cycles obtained by HST for network examples

(a) $m$-cycles obtained by HST in NSFNET; (b) $m$-cycles obtained by HST in ARPA2;

(c) $m$-cycles obtained by HST in SmallNet; (d) $m$-cycles obtained by HST in Bellcore. 


\section{B.2 M-cycles obtained by HST algorithm}

Table B.1 Fault localization results with $m$-cycles obtained by HST in NSFNET

\begin{tabular}{|c|c|c|c|c|c|c|c|c|}
\hline \multicolumn{8}{|c|}{ Alarm codes } & \multirow{2}{*}{$\begin{array}{l}\text { Faulty link } \\
\text { candidates }\end{array}$} \\
\hline $\mathrm{cl}$ & c2 & c3 & $\mathrm{c} 4$ & c5 & c6 & c7 & $\mathrm{c8}$ & \\
\hline 0 & 0 & 0 & 0 & 0 & 0 & 0 & 0 & Null \\
\hline 0 & 0 & 0 & 0 & 0 & 0 & 0 & 1 & $(10,14)$ \\
\hline 0 & 0 & 0 & 0 & 0 & 0 & 1 & 0 & $(9,14)$ \\
\hline 0 & 0 & 0 & 0 & 0 & 0 & 1 & 1 & $(12,14)$ \\
\hline 0 & 0 & 0 & 0 & 0 & 1 & 0 & 0 & $(6,11),(9,11)$ \\
\hline 0 & 0 & 0 & 0 & 1 & 0 & 0 & 0 & $(8,9)$ \\
\hline 0 & 0 & 0 & 0 & 1 & 1 & 1 & 0 & $(9,13)$ \\
\hline 0 & 0 & 0 & 1 & 0 & 0 & 0 & 0 & $(5,7),(7,8)$ \\
\hline 0 & 0 & 0 & 1 & 1 & 0 & 0 & 0 & $(2,8)$ \\
\hline 0 & 0 & 1 & 0 & 0 & 0 & 0 & 0 & $(4,10)$ \\
\hline 0 & 0 & 1 & 0 & 0 & 0 & 0 & 1 & $(10,13)$ \\
\hline 0 & 0 & 1 & 0 & 1 & 1 & 0 & 0 & $(6,12)$ \\
\hline 0 & 0 & 1 & 0 & 1 & 1 & 1 & 1 & $(12,13)$ \\
\hline 0 & 1 & 0 & 0 & 0 & 0 & 0 & 0 & $(1,4)$ \\
\hline 0 & 1 & 0 & 1 & 1 & 0 & 0 & 0 & $(3,6)$ \\
\hline 0 & 1 & 1 & 0 & 0 & 0 & 0 & 0 & $(4,5)$ \\
\hline 0 & 1 & 1 & 1 & 0 & 0 & 0 & 0 & $(5,6)$ \\
\hline 1 & 0 & 0 & 0 & 0 & 0 & 0 & 0 & $(1,2)$ \\
\hline 1 & 0 & 0 & 1 & 1 & 0 & 0 & 0 & $(2,3)$ \\
\hline 1 & 1 & 0 & 0 & 0 & 0 & 0 & 0 & $(1,3)$ \\
\hline \multicolumn{8}{|c|}{ Others } & N/A \\
\hline
\end{tabular}


Table B.2 Fault localization results with $m$-cycles obtained by HST in ARPA2

\begin{tabular}{|c|c|c|c|c|c|}
\hline \multicolumn{5}{|c|}{ Alarm codes } & \multirow{2}{*}{ Faulty link candidates } \\
\hline $\mathrm{cl}$ & $\mathrm{c} 2$ & c3 & $\mathrm{c4}$ & c5 & \\
\hline 0 & 0 & 0 & 0 & 0 & Null \\
\hline 0 & 0 & 0 & 0 & 1 & $(11,17),(16,19),(17,18),(18,21),(19,20),(20,21)$ \\
\hline 0 & 0 & 0 & 1 & 0 & $(6,15),(15,16)$ \\
\hline 0 & 0 & 0 & 1 & 1 & $(14,16)$ \\
\hline 0 & 0 & 1 & 0 & 0 & $(8,13),(13,14)$ \\
\hline 0 & 0 & 1 & $\mathbf{1}$ & 0 & $(8,9),(9,10),(10,11)$ \\
\hline 0 & 0 & 1 & 1 & 1 & $(11,12),(12,14)$ \\
\hline 0 & 1 & 0 & 0 & 0 & $(6,7),(7,8)$ \\
\hline 0 & $\mathbf{1}$ & 0 & 1 & 0 & $(1,8)$ \\
\hline 1 & 0 & 0 & 0 & 0 & $(1,4),(4,5),(5,6)$ \\
\hline 1 & $\mathbf{1}$ & 0 & 1 & 0 & $(1,2),(2,3),(3,6)$ \\
\hline \multicolumn{5}{|c|}{ Others } & N/A \\
\hline
\end{tabular}


Table B.3 Fault localization results with $m$-cycles obtained by HST in SmallNet

\begin{tabular}{|c|c|c|c|c|c|c|c|c|c|c|c|c|c|}
\hline \multicolumn{13}{|c|}{ Alarm codes } & \multirow{2}{*}{$\begin{array}{l}\text { Faulty link } \\
\text { candidates }\end{array}$} \\
\hline $\mathrm{cl}$ & $\mathrm{c} 2$ & c3 & $\mathrm{c4}$ & $\mathrm{c5}$ & c6 & c7 & c8 & c9 & $\mathrm{c} 10$ & $\mathrm{cll}$ & $\mathrm{cl} 2$ & $\mathrm{c} 13$ & \\
\hline 0 & 0 & 0 & 0 & 0 & 0 & 0 & 0 & 0 & 0 & 0 & 0 & 0 & Null \\
\hline 0 & 0 & 0 & 0 & 0 & 0 & 0 & 0 & 0 & 0 & 0 & 0 & 1 & $9-10$ \\
\hline 0 & 0 & 0 & 0 & 0 & 0 & 0 & 0 & 0 & 0 & 0 & $\mathbf{1}$ & 0 & $8-10$ \\
\hline 0 & 0 & 0 & 0 & 0 & 0 & 0 & 0 & 0 & 0 & 1 & 0 & 0 & $8-9$ \\
\hline 0 & 0 & 0 & 0 & 0 & 0 & 0 & 0 & 0 & 1 & 0 & 0 & 0 & $6-10$ \\
\hline 0 & 0 & 0 & 0 & 0 & 0 & 0 & 0 & 1 & 0 & 0 & 0 & 0 & $5-10$ \\
\hline 0 & 0 & 0 & 0 & 0 & 0 & 0 & 0 & 1 & 1 & 0 & $\mathbf{1}$ & 1 & $7-10$ \\
\hline 0 & 0 & 0 & 0 & 0 & 0 & 0 & 1 & 0 & 0 & 0 & 0 & 0 & $5-6$ \\
\hline 0 & 0 & 0 & 0 & 0 & 0 & 1 & 0 & 0 & 0 & 0 & 0 & 0 & $4-5$ \\
\hline 0 & 0 & 0 & 0 & 0 & 0 & 1 & 1 & 1 & 0 & 0 & 0 & 0 & $5-9$ \\
\hline 0 & 0 & 0 & 0 & 0 & 1 & 0 & 0 & 0 & 0 & 0 & 0 & 0 & $3-8$ \\
\hline 0 & 0 & 0 & 0 & 1 & 0 & 0 & 0 & 0 & 0 & 0 & 0 & 0 & $3-4$ \\
\hline 0 & 0 & 0 & 0 & 1 & 0 & 1 & 0 & 0 & 0 & 0 & 0 & 0 & $4-9$ \\
\hline 0 & 0 & 0 & 1 & 0 & 0 & 0 & 0 & 0 & 0 & 0 & 0 & 0 & $2-8$ \\
\hline 0 & 0 & 0 & 1 & 0 & 1 & 0 & 0 & 0 & 0 & 1 & 1 & 0 & $7-8$ \\
\hline 0 & 0 & 1 & 0 & 0 & 0 & 0 & 0 & 0 & 0 & 0 & 0 & 0 & $2-3$ \\
\hline 0 & 0 & 1 & 0 & 0 & 1 & 0 & 1 & 1 & 0 & 1 & 0 & 1 & $7-9$ \\
\hline 0 & 0 & 1 & 0 & 1 & 1 & 0 & 0 & 0 & 0 & 0 & 0 & 0 & $3-9$ \\
\hline 0 & 1 & 0 & 0 & 0 & 0 & 0 & 0 & 0 & 0 & 0 & 0 & 0 & $1-6$ \\
\hline 0 & 1 & 0 & 0 & 0 & 0 & 0 & 1 & 0 & 1 & 0 & 0 & 0 & $6-7$ \\
\hline 1 & 0 & 0 & 0 & 0 & 0 & 0 & 0 & 0 & 0 & 0 & 0 & 0 & $1-2$ \\
\hline 1 & 0 & 1 & 1 & 0 & 0 & 0 & 0 & 0 & 0 & 0 & 0 & 0 & $2-7$ \\
\hline 1 & 1 & 0 & 0 & 0 & 0 & 0 & 0 & 0 & 0 & 0 & 0 & 0 & $1-7$ \\
\hline & & & & & & & & & & & & & N/A \\
\hline
\end{tabular}


Table B.4 Fault localization results with $m$-cycles obtained by HST in Bellcore

\begin{tabular}{|c|c|c|c|c|c|c|c|c|c|c|c|c|c|c|}
\hline \multicolumn{14}{|c|}{ Alarm codes } & \multirow{2}{*}{$\begin{array}{c}\text { Faulty link } \\
\text { candidtes }\end{array}$} \\
\hline $\mathrm{cl}$ & $\mathrm{c} 2$ & c3 & $\mathrm{c} 4$ & c5 & c6 & c7 & $\mathrm{c} 8$ & c9 & c10 & c11 & $\mathrm{c} 12$ & $\mathrm{c} 13$ & $\mathrm{c} 14$ & \\
\hline 0 & 0 & 0 & 0 & 0 & 0 & 0 & 0 & 0 & 0 & 0 & 0 & 0 & 0 & Null \\
\hline 0 & 0 & 0 & 0 & 0 & 0 & 0 & 0 & 0 & 0 & 0 & 0 & 0 & 1 & $6-14,12-14$ \\
\hline 0 & 0 & 0 & 0 & 0 & 0 & 0 & 0 & 0 & 0 & 0 & 0 & 1 & 0 & $12-13$ \\
\hline 0 & 0 & 0 & 0 & 0 & 0 & 0 & 0 & 0 & 0 & 0 & 1 & 0 & 0 & $9-11$ \\
\hline 0 & 0 & 0 & 0 & 0 & 0 & 0 & 0 & 0 & 0 & 1 & 0 & 0 & 0 & $9-10$ \\
\hline 0 & 0 & 0 & 0 & 0 & 0 & 0 & 0 & 0 & 1 & 0 & 0 & 0 & 0 & $8-11$ \\
\hline 0 & 0 & 0 & 0 & 0 & 0 & 0 & 0 & 0 & 1 & 0 & 1 & 0 & 0 & $2-11$ \\
\hline 0 & 0 & 0 & 0 & 0 & 0 & 0 & 0 & 1 & 0 & 0 & 0 & 0 & 0 & $7-12$ \\
\hline 0 & 0 & 0 & 0 & 0 & 0 & 0 & 1 & 0 & 0 & 0 & 0 & 0 & 0 & $5-15,6-15$ \\
\hline 0 & 0 & 0 & 0 & 0 & 0 & 1 & 0 & 0 & 0 & 0 & 0 & 0 & 0 & $6-12$ \\
\hline 0 & 0 & 0 & 0 & 0 & 0 & 1 & 0 & 1 & 0 & 0 & 0 & 1 & 1 & $8-12$ \\
\hline 0 & 0 & 0 & 0 & 0 & 1 & 0 & 0 & 0 & 0 & 0 & 0 & 0 & 0 & $6-7$ \\
\hline 0 & 0 & 0 & 0 & 0 & 1 & 0 & 0 & 1 & 0 & 0 & 0 & 0 & 0 & $7-8$ \\
\hline 0 & 0 & 0 & 0 & 1 & 0 & 0 & 0 & 0 & 0 & 0 & 0 & 0 & 0 & $4-13$ \\
\hline 0 & 0 & 0 & 1 & 0 & 0 & 0 & 0 & 0 & 0 & 0 & 0 & 0 & 0 & $4-5$ \\
\hline 0 & 0 & 0 & 1 & 0 & 0 & 0 & 1 & 0 & 0 & 0 & 0 & 0 & 0 & $5-6$ \\
\hline 0 & 0 & 0 & 1 & 0 & 1 & 1 & 0 & 0 & 0 & 0 & 0 & 0 & 1 & $3-6$ \\
\hline 0 & 0 & 0 & 1 & 1 & 0 & 0 & 0 & 0 & 0 & 0 & 0 & 0 & 0 & $3-4$ \\
\hline 0 & 0 & 1 & 0 & 0 & 0 & 0 & 0 & 0 & 0 & 0 & 0 & 0 & 0 & $3-13$ \\
\hline 0 & 0 & 1 & 0 & 1 & 0 & 0 & 0 & 0 & 0 & 0 & 0 & 1 & 0 & $2-13$ \\
\hline 0 & 0 & 1 & 0 & 1 & 1 & 1 & 0 & 0 & 0 & 0 & 0 & 0 & 1 & $2-3$ \\
\hline 0 & 1 & 0 & 0 & 0 & 0 & 0 & 0 & 0 & 0 & 0 & 0 & 0 & 0 & $1-10$ \\
\hline 0 & 1 & 0 & 0 & 0 & 0 & 0 & 0 & 0 & 0 & 1 & 0 & 0 & 0 & $2-10$ \\
\hline 1 & 0 & 0 & 0 & 0 & 0 & 0 & 0 & 0 & 0 & 0 & 0 & 0 & 0 & $1-9$ \\
\hline 1 & 0 & 0 & 0 & 0 & 0 & 0 & 0 & 0 & 0 & 1 & 1 & 0 & 0 & $8-9$ \\
\hline 1 & 0 & 0 & 0 & 0 & 1 & 1 & 0 & 0 & 1 & 1 & 1 & 1 & 1 & $2-8$ \\
\hline 1 & 1 & 0 & 0 & 0 & 0 & 0 & 0 & 0 & 0 & 0 & 0 & 0 & 0 & $1-2$ \\
\hline & & & & & & & & & & & & & & N/A \\
\hline
\end{tabular}




\section{Appendix C}

\section{vCCCP Algorithm Explanation}

This appendix lists the tables of candidate cycles produced by SPCC, as well as the
checking-matrix for verifying the existence of feasible solutions of the vCCCP problem in the network example given in Figure 6.5. Table C.3 - C.6 list optimal solutions obtained by the two-phase vCCCP algorithm for network examples shown in Figure 6.7: SmallNet, Bellcore, NSFNET, and ARPA2.

C.1 SPCC candidate cycles and checking-matrix for the example in Figure 6.5 
Table C. 1 Candidate cycles obtained by SPCC for the network example in Figure 6.5

\begin{tabular}{ccc}
$m$-cycle \# & $m$-cycle & $m$-cycle length (Weight) \\
1 & $1-2-6-1$ & 3 \\
2 & $1-5-6-1$ & 3 \\
3 & $2-3-6-2$ & 3 \\
4 & $3-4-6-3$ & 3 \\
5 & $4-5-6-4$ & 4 \\
6 & $1-2-3-6-1$ & 4 \\
7 & $1-2-6-5-1$ & 4 \\
8 & $1-5-4-6-1$ & 4 \\
9 & $2-3-4-6-2$ & 4 \\
10 & $3-4-5-6-3$ & 5 \\
11 & $1-2-3-4-5-1$ & 5 \\
12 & $1-2-3-6-5-1$ & 5 \\
13 & $1-2-3-4-6-1$ & 5 \\
14 & $1-5-4-3-6-1$ & 5 \\
15 & $2-3-4-5-6-2$ & 5 \\
16 & $1-2-6-4-5-1$ & \\
\hline
\end{tabular}

$-180-$ 
Table C.2 The checking-matrix for verifying the existence of feasible solutions of vCCCP problem in the example network in Figure 6.5

\begin{tabular}{ccccccccccc}
$m$-cycle & $(1-2)$ & $(1-5)$ & $(1-6)$ & $(2-3)$ & $(2-6)$ & $(3-4)$ & $(3-6)$ & $(4-5)$ & $(4-6)$ & $(5-6)$ \\
1 & 1 & & 1 & & 1 & & & & & \\
2 & & 1 & 1 & & & & & & & 1 \\
3 & & & & 1 & 1 & & 1 & & & \\
4 & & & & & & 1 & 1 & & 1 & \\
5 & & & & & & & & 1 & 1 & 1 \\
6 & 1 & & 1 & 1 & & & 1 & & & \\
7 & 1 & 1 & & & 1 & & & & & 1 \\
8 & & 1 & 1 & & & & & 1 & 1 & \\
9 & & & & 1 & 1 & 1 & & & 1 & \\
10 & & & & & & 1 & 1 & 1 & & 1 \\
11 & 1 & 1 & & 1 & & 1 & & 1 & & \\
12 & 1 & 1 & & 1 & & & 1 & & & 1 \\
13 & 1 & & 1 & 1 & & 1 & & & 1 & \\
14 & & 1 & 1 & & & 1 & 1 & 1 & & \\
15 & & & & 1 & 1 & 1 & & 1 & & 1 \\
16 & 1 & 1 & & & 1 & & & 1 & 1 \\
\hline
\end{tabular}

- $181-$ 


\section{C.2 Re-number nodes in typical network examples with chain removal}

Nodes in network examples Bellcore, NSFNET, and ARPA2 are re-numbered and shown in Figures C.1 - C.3, for the convenience of vCCCP agorithm implementation (in MicroSoft ${ }^{\circledR}$ Visual $\mathrm{C}++6.0$ ). All results of the vCCCP algorithm for these examples listed in Tables C.3 - C.6 are based on such node numbers.
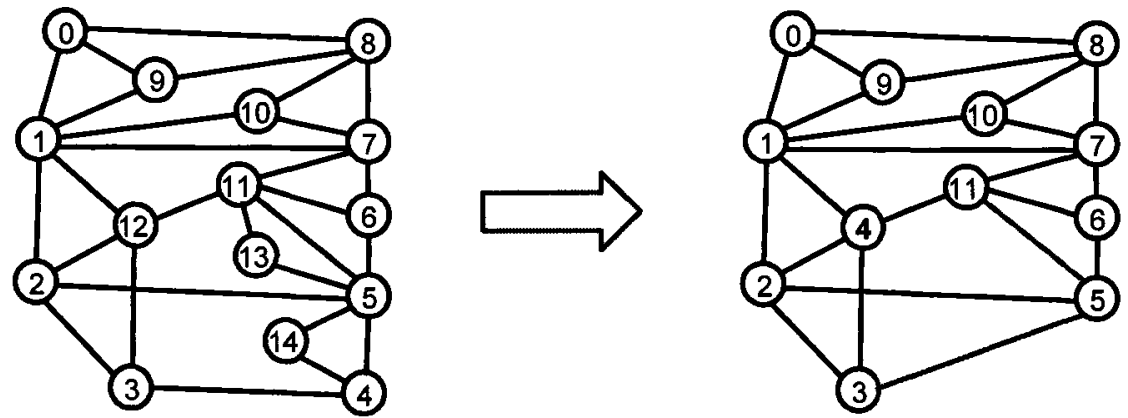

Removed edges: $3-4,4-5,4-14,5-14 \rightarrow 3-5 ; \quad 5-13,11-13,5-11 \rightarrow 5-11$

Figure C.1 Re-numbered nodes in Bellcore after move degree- 2 chains
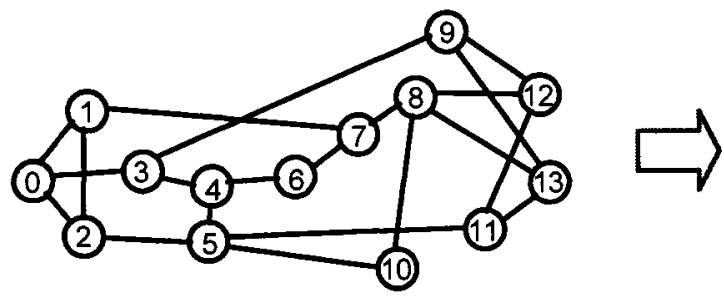

Removed edges: $5-10,10-8 \rightarrow 5-8 ; \quad 4-6,6-7 \rightarrow 4-7$

Figure C.2 Re-numbered nodes in NSFNET after move degree-2 chains 


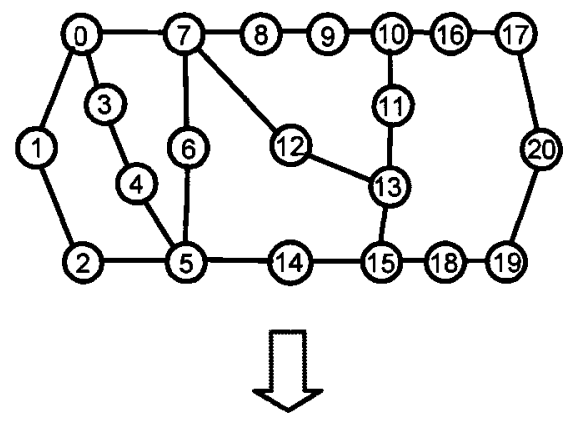

Removed edges (step 1):

$0-1,1-2,2-5 \rightarrow 0-5$

$0-3,3-4,4-5 \rightarrow 0-5$ (duplicated)

5-6, 6-7 $\rightarrow 5-7$

$7-12,12-13 \rightarrow 7-13$

$5-14,14-15 \rightarrow 5-15$

$7-8,8-9,9-10 \rightarrow 7-10$

$10-11,11-13 \rightarrow 10-13$

$10-16,16-17,17-20,20-19,19-18,18-15 \rightarrow 10-15$
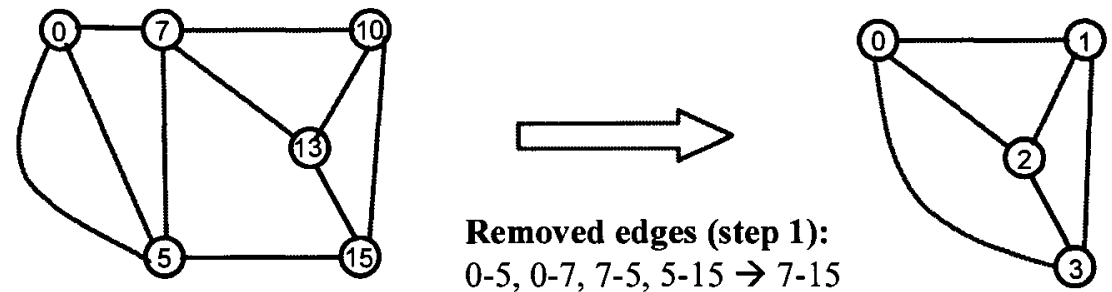

Figure C.3 Re-numbered nodes in ARPA2 after move degree-2 chains 


\section{C.3 Results of vCCCP algorithm for typical network}

\section{examples}

Table C.3 Optimal solutions for SmallNet obtained by the two-phase vCCCP algorithm

\begin{tabular}{|c|c|c|c|}
\hline$m$-cycle & Solution \#1 & Solution \#2 & Solution \#3 \\
\hline 1 & $\begin{array}{llll}0 & 6 & 1 & 0\end{array}$ & $\begin{array}{llll}0 & 6 & 1 & 0\end{array}$ & $\begin{array}{llll}0 & 6 & 1 & 0\end{array}$ \\
\hline 2 & 0650 & 0650 & 0650 \\
\hline 3 & 1721 & 1721 & 1721 \\
\hline 4 & 1761 & 1761 & 1761 \\
\hline 5 & 2832 & 2832 & 2832 \\
\hline 6 & 2872 & 2872 & 2872 \\
\hline 7 & 3843 & 3843 & 3843 \\
\hline 8 & 4954 & 4954 & 4954 \\
\hline 9 & 4984 & 4984 & 4984 \\
\hline 10 & 5965 & 5965 & 5965 \\
\hline 11 & 6876 & 6976 & 6986 \\
\hline 12 & 6976 & 6986 & 7987 \\
\hline
\end{tabular}

Table C.4 Optimal solutions for Bellcore obtained by the two-phase vCCCP algorithm

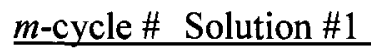
Solution \#2

$\begin{array}{lllllllll}1 & 0 & 9 & 1 & 0 & 0 \\ 2 & 0 & 9 & 8 & 0 & 0 & 9 & 1 & 0 \\ 3 & 1 & 4 & 2 & 1 & 0 & 9 & 8 & 0 \\ 4 & 1 & 10 & 7 & 1 & 1 & 4 & 2 & 1 \\ 5 & 2 & 4 & 3 & 2 & 1 & 10 & 7 & 1 \\ 6 & 2 & 5 & 3 & 2 & 2 & 4 & 3 & 2 \\ 7 & 5 & 11 & 6 & 5 & 2 & 5 & 3 & 2 \\ & & 5 & 11 & 6 & 5\end{array}$

$-184-$ 


\begin{tabular}{|c|c|c|}
\hline 8 & 61176 & 61176 \\
\hline 9 & $7 \quad 1087$ & $\begin{array}{llll}7 & 10 & 8 & 7\end{array}$ \\
\hline 10 & 171141 & 125671 \\
\hline 11 & $\begin{array}{lllllll}87 & 6 & 5 & 2 & 1 & 9 & 8\end{array}$ & $\begin{array}{lllllll}0 & 8 & 7 & 11 & 4 & 1 & 0 \\
\end{array}$ \\
\hline
\end{tabular}

Table C.5 Optimal solutions for NSFNET obtained by the two-phase vCCCP algorithm

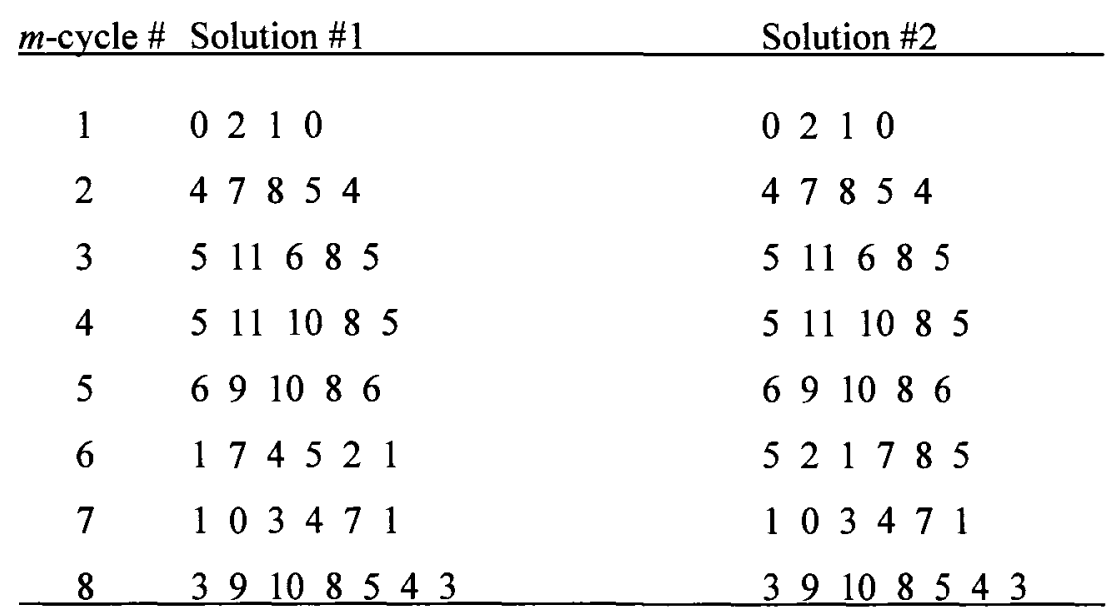

Table C.6 Optimal solutions for ARPA2 obtained by the two-phase vCCCP algorithm

\begin{tabular}{|c|c|c|}
\hline$m$-cycle \# & Solution \#1 & Solution \#2 \\
\hline 1 & 0210 & $\begin{array}{llll}0 & 2 & 1 & 0\end{array}$ \\
\hline 2 & $\begin{array}{llll}0 & 3 & 1 & 0\end{array}$ & $\begin{array}{llll}0 & 3 & 2 & 0\end{array}$ \\
\hline 3 & $\begin{array}{l}0320 \\
\end{array}$ & 1321 \\
\hline
\end{tabular}

$-185-$ 


\section{Appendix D}

\section{Empirical Study of vCCCP Algorithm}

This appendix lists the tables of randomly generated network examples in the
format of from- and to-nodes. Optimal solutions obtained by the B\&B vCCCP algorithm are in tables following each network example.

Table D.1 Randomly generated network example (1) with 13 nodes and 20 links

\begin{tabular}{cc} 
From-node & To-node \\
\hline 0 & 7 \\
0 & 5 \\
0 & 2 \\
1 & 9 \\
1 & 8 \\
1 & 10 \\
2 & 12 \\
2 & 3 \\
3 & 9 \\
3 & 6 \\
4 & 10 \\
4 & 7 \\
4 & 6 \\
5 & 12 \\
5 & 8 \\
6 & 11 \\
7 & 9 \\
8 & 11 \\
10 & 12 \\
10 & 11 \\
\hline
\end{tabular}

- $186-$ 
Table D.2 Optimal solutions of -cycles for randomly generated network example (1)

\begin{tabular}{|c|c|c|}
\hline$m$-cycle \# & Solution \#1 & Solution \#2 \\
\hline 1 & $\begin{array}{lllll}0 & 5 & 12 & 2 & 0\end{array}$ & $\begin{array}{lllll}0 & 5 & 12 & 2 & 0\end{array}$ \\
\hline 2 & $\begin{array}{lllll}1 & 10 & 11 & 8 & 1\end{array}$ & $\begin{array}{lllll}1 & 10 & 11 & 8 & 1\end{array}$ \\
\hline 3 & $\begin{array}{lllll}4 & 10 & 11 & 6 & 4\end{array}$ & 4101164 \\
\hline 4 & 397463 & 397463 \\
\hline 5 & 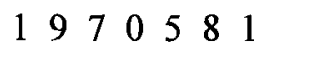 & $\begin{array}{lllllll}3 & 2 & 12 & 10 & 4 & 6 & 3\end{array}$ \\
\hline 6 & $\begin{array}{lllllll}3 & 2 & 12 & 10 & 1 & 9 & 3\end{array}$ & $\begin{array}{llllllll}0 & 5 & 8 & 1 & 9 & 3 & 2 & 0\end{array}$ \\
\hline 7 & $\begin{array}{lllllll}4 & 10 & 12 & 5 & 0 & 7 & 4 \\
\end{array}$ & $\begin{array}{llllllll}4 & 7 & 0 & 5 & 8 & 11 & 6 & 4\end{array}$ \\
\hline
\end{tabular}

- 187 - 
Table D.3 Randomly generated network example (2) with 18 nodes and 37 links

\begin{tabular}{|c|c|}
\hline From-node & To-node \\
\hline 0 & 14 \\
\hline 0 & 12 \\
\hline 0 & 8 \\
\hline 1 & 11 \\
\hline 1 & 9 \\
\hline 1 & 7 \\
\hline 1 & 2 \\
\hline 2 & 14 \\
\hline 2 & 7 \\
\hline 3 & 14 \\
\hline 3 & 8 \\
\hline 3 & 5 \\
\hline 3 & 4 \\
\hline 4 & 15 \\
\hline 4 & 12 \\
\hline 4 & 10 \\
\hline 5 & 13 \\
\hline 5 & 8 \\
\hline 6 & 13 \\
\hline 6 & 10 \\
\hline 6 & 9 \\
\hline 7 & 17 \\
\hline 7 & 14 \\
\hline 7 & 11 \\
\hline 8 & 10 \\
\hline 9 & 17 \\
\hline 9 & 15 \\
\hline 9 & 10 \\
\hline 10 & 16 \\
\hline 11 & 17 \\
\hline 12 & 16 \\
\hline 12 & 14 \\
\hline 13 & 15 \\
\hline 9 & 16 \\
\hline
\end{tabular}

- 188 - 
Table D.4 Optimal solutions of -cycles for randomly generated network example (2)

\begin{tabular}{|c|c|c|c|}
\hline$m$-cycle & Solution \#1 & Solution \#2 & Solution \#3 \\
\hline 1 & $\begin{array}{llll}0 & 14 & 12 & 0\end{array}$ & $\begin{array}{llll}0 & 14 & 12 & 0\end{array}$ & $\begin{array}{llll}0 & 14 & 12 & 0\end{array}$ \\
\hline 2 & 1721 & 1721 & 1721 \\
\hline 3 & 11171 & 11171 & 11171 \\
\hline 4 & 21472 & 21472 & 21472 \\
\hline 5 & 3853 & 3853 & 3853 \\
\hline 6 & 61096 & 61096 & 61096 \\
\hline 7 & $\begin{array}{lllll}7 & 17 & 11 & 7\end{array}$ & $\begin{array}{llll}7 & 17 & 11 & 7\end{array}$ & $\begin{array}{lllll}7 & 17 & 11 & 7\end{array}$ \\
\hline 8 & 916109 & $9 \quad 16109$ & 916109 \\
\hline 9 & 087740 & 087740 & $\begin{array}{lllll}0 & 8 & 7 & 14 & 0\end{array}$ \\
\hline 10 & 211172 & 211172 & 211172 \\
\hline 11 & $\begin{array}{lllll}2 & 1 & 7 & 14 & 2\end{array}$ & $\begin{array}{lllll}2 & 1 & 7 & 14 & 2\end{array}$ & $\begin{array}{lllll}2 & 1 & 7 & 14 & 2\end{array}$ \\
\hline 12 & $\begin{array}{lllll}3 & 5 & 12 & 4 & 3\end{array}$ & $\begin{array}{lllll}3 & 5 & 12 & 4 & 3\end{array}$ & $\begin{array}{lllll}3 & 5 & 12 & 4 & 3\end{array}$ \\
\hline 13 & $\begin{array}{lllll}3 & 8 & 10 & 4 & 3\end{array}$ & 381043 & $\begin{array}{lllll}3 & 8 & 10 & 4 & 3\end{array}$ \\
\hline 14 & $\begin{array}{lllll}3 & 14 & 12 & 4 & 3\end{array}$ & $\begin{array}{lllll}3 & 14 & 12 & 4 & 3\end{array}$ & $\begin{array}{lllll}3 & 14 & 12 & 4 & 3\end{array}$ \\
\hline 15 & $\begin{array}{lllll}3 & 14 & 12 & 5 & 3\end{array}$ & $\begin{array}{lllll}3 & 14 & 12 & 5 & 3\end{array}$ & $\begin{array}{lllll}3 & 14 & 12 & 5 & 3\end{array}$ \\
\hline 16 & $\begin{array}{lllll}3 & 14 & 0 & 8 & 3\end{array}$ & $\begin{array}{lllll}3 & 14 & 0 & 8 & 3\end{array}$ & $\begin{array}{lllll}3 & 14 & 0 & 8 & 3\end{array}$ \\
\hline 17 & $\begin{array}{lllll}3 & 14 & 7 & 8 & 3\end{array}$ & 314783 & $\begin{array}{lllll}3 & 14 & 7 & 8 & 3\end{array}$ \\
\hline 18 & $4 \quad 159104$ & $4 \quad 159104$ & $\begin{array}{lllll}4 & 15 & 9 & 10 & 4\end{array}$ \\
\hline 19 & $\begin{array}{lllll}4 & 12 & 16 & 10 & 4\end{array}$ & $\begin{array}{lllll}4 & 12 & 16 & 10 & 4\end{array}$ & $\begin{array}{lllll}4 & 12 & 16 & 10 & 4\end{array}$ \\
\hline 20 & $\begin{array}{lllll}0 & 12 & 5 & 8 & 0\end{array}$ & $\begin{array}{lllll}0 & 12 & 5 & 8 & 0\end{array}$ & $\begin{array}{lllll}0 & 12 & 5 & 8 & 0\end{array}$ \\
\hline 21 & $\begin{array}{lllll}6 & 13 & 15 & 9 & 6\end{array}$ & $\begin{array}{lllll}6 & 13 & 159\end{array}$ & $\begin{array}{lllll}6 & 13 & 15 & 9 & 6\end{array}$ \\
\hline 22 & 6101696 & 6101696 & $\begin{array}{lll}6 & 10 & 169\end{array}$ \\
\hline 23 & 191671 & 191671 & 191671 \\
\hline 24 & 191771 & $\begin{array}{lllll}7 & 179 & 16 & 7\end{array}$ & $111 \quad 1791$ \\
\hline 25 & $\begin{array}{llllll}3 & 4 & 15 & 13 & 5 & 3\end{array}$ & $\begin{array}{llllll}3 & 4 & 15 & 13 & 5 & 3\end{array}$ & $\begin{array}{llllll}3 & 4 & 15 & 13 & 5 & 3 \\
\end{array}$ \\
\hline
\end{tabular}

$-189-$ 


\begin{tabular}{|c|c|c|}
\hline 1 & $\begin{array}{lllll}0 & 14 & 12 & 0\end{array}$ & $\begin{array}{llll}0 & 14 & 12 & 0\end{array}$ \\
\hline 2 & 1721 & 1721 \\
\hline 3 & $\begin{array}{llll}1 & 11 & 7 & 1\end{array}$ & $\begin{array}{llll}1 & 11 & 7 & 1\end{array}$ \\
\hline 4 & 21472 & 21472 \\
\hline 5 & 3853 & 3853 \\
\hline 6 & 61096 & 61096 \\
\hline 7 & $\begin{array}{llll}7 & 17 & 11 & 7\end{array}$ & $\begin{array}{llll}7 & 17 & 11 & 7\end{array}$ \\
\hline 8 & $9 \quad 16 \quad 109$ & $9 \quad 16 \quad 109$ \\
\hline 9 & $\begin{array}{llllll}0 & 8 & 7 & 14 & 0\end{array}$ & $\begin{array}{lllll}0 & 8 & 7 & 14 & 0\end{array}$ \\
\hline 10 & $\begin{array}{lllll}2 & 1 & 11 & 7 & 2\end{array}$ & 2111172 \\
\hline 11 & $\begin{array}{lllll}2 & 1 & 7 & 14 & 2\end{array}$ & $\begin{array}{lllll}2 & 1 & 7 & 14 & 2\end{array}$ \\
\hline 12 & $\begin{array}{lllll}3 & 5 & 12 & 4 & 3\end{array}$ & $\begin{array}{lllll}3 & 5 & 12 & 4 & 3\end{array}$ \\
\hline 13 & $\begin{array}{lllll}3 & 8 & 10 & 4 & 3\end{array}$ & $\begin{array}{lllll}3 & 8 & 10 & 4 & 3\end{array}$ \\
\hline 14 & $\begin{array}{lllll}3 & 14 & 12 & 4 & 3\end{array}$ & $\begin{array}{lllll}3 & 14 & 12 & 4 & 3\end{array}$ \\
\hline 15 & $\begin{array}{lllll}3 & 14 & 12 & 5 & 3\end{array}$ & $\begin{array}{lllll}3 & 14 & 12 & 5 & 3\end{array}$ \\
\hline 16 & $\begin{array}{lllll}3 & 14 & 0 & 8 & 3\end{array}$ & 314083 \\
\hline 17 & $\begin{array}{lllll}3 & 14 & 7 & 8 & 3\end{array}$ & 314783 \\
\hline 18 & $\begin{array}{lllll}4 & 15 & 9 & 10 & 4\end{array}$ & $\begin{array}{lllll}4 & 15 & 9 & 10 & 4\end{array}$ \\
\hline 19 & $\begin{array}{lllll}4 & 12 & 16 & 10 & 4\end{array}$ & $\begin{array}{lllll}4 & 12 & 16 & 10 & 4\end{array}$ \\
\hline 20 & $\begin{array}{lllll}0 & 12 & 5 & 8 & 0\end{array}$ & $\begin{array}{lllll}0 & 12 & 5 & 8 & 0\end{array}$ \\
\hline 21 & $\begin{array}{lllll}6 & 13 & 15 & 9 & 6\end{array}$ & $\begin{array}{lllll}6 & 13 & 15 & 9 & 6\end{array}$ \\
\hline 22 & $\begin{array}{lllll}6 & 10 & 16 & 9 & 6\end{array}$ & $\begin{array}{lllll}6 & 10 & 16 & 9 & 6\end{array}$ \\
\hline 23 & 191771 & 7179167 \\
\hline 24 & $7 \quad 179167$ & $\begin{array}{lllll}1 & 11 & 17 & 9 & 1\end{array}$ \\
\hline 25 & $\begin{array}{llllll}3 & 4 & 15 & 13 & 5 & 3\end{array}$ & $\begin{array}{llllll}3 & 4 & 15 & 13 & 5 & 3\end{array}$ \\
\hline
\end{tabular}

$-190-$ 
Table D.5 Randomly generated network example (3) with 21 nodes and 37 links

\begin{tabular}{|c|c|}
\hline From-node & To-node \\
\hline 0 & 18 \\
\hline 0 & 17 \\
\hline 0 & 12 \\
\hline 0 & 5 \\
\hline 1 & 20 \\
\hline 1 & 14 \\
\hline 2 & 19 \\
\hline 2 & 14 \\
\hline 2 & 8 \\
\hline 3 & 8 \\
\hline 3 & 7 \\
\hline 4 & 16 \\
\hline 4 & 13 \\
\hline 5 & 16 \\
\hline 6 & 20 \\
\hline 6 & 15 \\
\hline 7 & 11 \\
\hline 8 & 18 \\
\hline 9 & 17 \\
\hline 9 & 15 \\
\hline 9 & 13 \\
\hline 10 & 18 \\
\hline 11 & 20 \\
\hline 12 & 15 \\
\hline 13 & 19 \\
\hline 16 & 17 \\
\hline 17 & 19 \\
\hline 15 & 16 \\
\hline 7 & 10 \\
\hline 7 & 12 \\
\hline 6 & 12 \\
\hline 4 & 5 \\
\hline 1 & 13 \\
\hline 5 & 7 \\
\hline 3 & 10 \\
\hline 14 & 18 \\
\hline 0 & 11 \\
\hline
\end{tabular}

- 191 - 
Table D.6 Optimal solutions of -cycles for randomly generated network example (3) $m$-cycle \# Solution \#1 Solution \#2

\begin{tabular}{|c|c|c|}
\hline 1 & 31073 & $\begin{array}{llll}3 & 10 & 7 & 3\end{array}$ \\
\hline 2 & 41654 & 41654 \\
\hline 3 & $6 \quad 15126$ & $\begin{array}{llll}6 & 15 & 12 & 6\end{array}$ \\
\hline 4 & $\begin{array}{lllll}2 & 8 & 18 & 14 & 2\end{array}$ & $\begin{array}{lllll}2 & 8 & 18 & 14 & 2\end{array}$ \\
\hline 5 & $\begin{array}{lllll}0 & 11 & 7 & 5 & 0\end{array}$ & $\begin{array}{lllll}0 & 11 & 7 & 5 & 0\end{array}$ \\
\hline 6 & $\begin{array}{lllll}3 & 10 & 18 & 8 & 3\end{array}$ & $\begin{array}{lllll}3 & 10 & 18 & 8 & 3\end{array}$ \\
\hline 7 & $\begin{array}{lllll}0 & 12 & 7 & 5 & 0\end{array}$ & $\begin{array}{lllll}0 & 12 & 7 & 5 & 0\end{array}$ \\
\hline 8 & $\begin{array}{lllll}0 & 17 & 16 & 5 & 0\end{array}$ & $\begin{array}{lllll}0 & 17 & 16 & 5 & 0\end{array}$ \\
\hline 9 & $\begin{array}{lllll}0 & 12 & 7 & 11 & 0\end{array}$ & $\begin{array}{lllll}0 & 12 & 7 & 11 & 0\end{array}$ \\
\hline 10 & $\begin{array}{lllll}9 & 17 & 19 & 13 & 9\end{array}$ & $\begin{array}{lllll}9 & 17 & 19 & 13 & 9\end{array}$ \\
\hline 11 & $\begin{array}{lllll}9 & 17 & 16 & 15 & 9\end{array}$ & $\begin{array}{lllll}9 & 17 & 16 & 15 & 9\end{array}$ \\
\hline 12 & $\begin{array}{llllll}4 & 5 & 0 & 17 & 16 & 4\end{array}$ & $\begin{array}{llllll}4 & 5 & 0 & 17 & 16 & 4\end{array}$ \\
\hline 13 & $\begin{array}{llllll}4 & 13 & 19 & 17 & 16 & 4\end{array}$ & $\begin{array}{llllll}4 & 13 & 19 & 17 & 16 & 4\end{array}$ \\
\hline 14 & $\begin{array}{llllll}0 & 12 & 15 & 9 & 17 & 0\end{array}$ & $\begin{array}{llllll}0 & 12 & 15 & 9 & 17 & 0\end{array}$ \\
\hline 15 & $\begin{array}{llllll}5 & 0 & 18 & 10 & 7 & 5\end{array}$ & $\begin{array}{llllll}5 & 0 & 18 & 10 & 7 & 5\end{array}$ \\
\hline 16 & $\begin{array}{llllll}5 & 16 & 15 & 12 & 7 & 5\end{array}$ & $\begin{array}{llllll}5 & 16 & 15 & 12 & 7 & 5\end{array}$ \\
\hline 17 & $\begin{array}{llllll}5 & 0 & 12 & 15 & 16 & 5\end{array}$ & $\begin{array}{llllll}5 & 0 & 12 & 15 & 16 & 5\end{array}$ \\
\hline 18 & $\begin{array}{llllll}0 & 11 & 7 & 10 & 18 & 0\end{array}$ & $\begin{array}{llllll}0 & 11 & 7 & 10 & 18 & 0\end{array}$ \\
\hline 19 & $\begin{array}{llllll}6 & 20 & 11 & 0 & 12 & 6\end{array}$ & $\begin{array}{llllll}6 & 20 & 11 & 0 & 12 & 6\end{array}$ \\
\hline 20 & $\begin{array}{llllll}6 & 20 & 11 & 7 & 12 & 6\end{array}$ & $\begin{array}{llllll}6 & 20 & 11 & 7 & 12 & 6\end{array}$ \\
\hline 21 & $\begin{array}{llllll}0 & 12 & 7 & 10 & 18 & 0\end{array}$ & $\begin{array}{llllll}0 & 12 & 7 & 10 & 18 & 0\end{array}$ \\
\hline 22 & $\begin{array}{llllll}1 & 14 & 2 & 19 & 13 & 1\end{array}$ & $\begin{array}{llllll}1 & 14 & 2 & 19 & 13 & 1\end{array}$ \\
\hline 23 & $\begin{array}{llllll}8 & 3 & 7 & 10 & 18 & 8\end{array}$ & $\begin{array}{llllll}8 & 3 & 7 & 10 & 18 & 8\end{array}$ \\
\hline 24 & $\begin{array}{llllll}9 & 15 & 16 & 4 & 13 & 9\end{array}$ & $\begin{array}{llllll}9 & 15 & 16 & 4 & 13 & 9\end{array}$ \\
\hline 25 & $913 \quad 4 \quad 16179$ & $\begin{array}{llllll}9 & 13 & 4 & 16 & 17 & 9\end{array}$ \\
\hline 26 & $\begin{array}{llllll}16 & 15 & 12 & 0 & 17 & 16\end{array}$ & $\begin{array}{llllll}16 & 15 & 12 & 0 & 17 & 16\end{array}$ \\
\hline 27 & $\begin{array}{lllllll}3 & 7 & 5 & 0 & 18 & 10 & 3\end{array}$ & $\begin{array}{lllllll}3 & 7 & 5 & 0 & 18 & 10 & 3\end{array}$ \\
\hline 28 & $\begin{array}{lllllll}3 & 8 & 2 & 14 & 18 & 10 & 3\end{array}$ & $\begin{array}{lllllll}3 & 8 & 2 & 14 & 18 & 10 & 3\end{array}$ \\
\hline 29 & $\begin{array}{llllllll}7 & 3 & 8 & 18 & 0 & 11 & 7\end{array}$ & $\begin{array}{llllllll}7 & 3 & 8 & 18 & 0 & 11 & 7\end{array}$ \\
\hline 30 & $\begin{array}{lllllll}0 & 11 & 20 & 1 & 14 & 18 & 0\end{array}$ & $\begin{array}{lllllll}0 & 17 & 19 & 2 & 8 & 18 & 0\end{array}$ \\
\hline 31 & $017 \quad 1928818 \quad 0$ & $\begin{array}{lllllll}12 & 20 & 6 & 15 & 9 & 13 & 1\end{array}$ \\
\hline
\end{tabular}

$-192-$ 
Table D.7 Randomly generated network example (4) with 30 nodes and 61 links

\begin{tabular}{|c|c|c|c|}
\hline From-node & To-node & From-node & To-node \\
\hline 0 & 16 & 15 & 23 \\
\hline 0 & 14 & 15 & 20 \\
\hline 0 & 8 & 17 & 26 \\
\hline 1 & 27 & 17 & 19 \\
\hline 1 & 17 & 17 & 18 \\
\hline 1 & 6 & 18 & 28 \\
\hline 2 & 29 & 18 & 20 \\
\hline 2 & 20 & 19 & 26 \\
\hline 3 & 24 & 19 & 24 \\
\hline 3 & 9 & 21 & 29 \\
\hline 3 & 6 & 21 & 26 \\
\hline 4 & 25 & 23 & 24 \\
\hline 4 & 18 & 24 & 29 \\
\hline 4 & 10 & 25 & 28 \\
\hline 5 & 29 & 26 & 27 \\
\hline 5 & 25 & 4 & 7 \\
\hline 5 & 22 & 1 & 10 \\
\hline 5 & 13 & 19 & 21 \\
\hline 5 & 6 & 3 & 16 \\
\hline 6 & 19 & 7 & 24 \\
\hline 7 & 11 & 2 & 19 \\
\hline 8 & 23 & & \\
\hline 8 & 17 & & \\
\hline 8 & 9 & & \\
\hline 9 & 11 & & \\
\hline 9 & 10 & & \\
\hline 10 & 27 & & \\
\hline 10 & 22 & & \\
\hline 10 & 21 & & \\
\hline 11 & 29 & & \\
\hline 11 & 26 & & \\
\hline 11 & 14 & & \\
\hline 11 & 12 & & \\
\hline 12 & 27 & & \\
\hline 12 & 25 & & \\
\hline 13 & 28 & & \\
\hline 13 & 22 & & \\
\hline 13 & 20 & & \\
\hline 13 & 15 & & \\
\hline 14 & 16 & & \\
\hline
\end{tabular}

- 193 - 
Table D.8 Optimal solutions of -cycles for randomly generated network example (4)

m-cycle \# Solution \#1

\begin{tabular}{|c|c|}
\hline 1 & $\begin{array}{lllll}0 & 16 & 14 & 0\end{array}$ \\
\hline 2 & $\begin{array}{llll}1 & 27 & 10 & 1\end{array}$ \\
\hline 3 & $\begin{array}{llll}5 & 22 & 13 & 5\end{array}$ \\
\hline 4 & $\begin{array}{llll}13 & 20 & 15 & 13\end{array}$ \\
\hline 5 & $\begin{array}{llll}17 & 26 & 19 & 17\end{array}$ \\
\hline 6 & $192621 \quad 19$ \\
\hline 7 & $\begin{array}{lllll}1 & 27 & 26 & 17 & 1\end{array}$ \\
\hline 8 & $\begin{array}{lllll}5 & 13 & 28 & 25 & 5\end{array}$ \\
\hline 9 & $\begin{array}{lllll}6 & 1 & 17 & 19 & 6\end{array}$ \\
\hline 10 & $\begin{array}{lllll}6 & 3 & 24 & 19 & 6\end{array}$ \\
\hline 11 & 72429117 \\
\hline 12 & $\begin{array}{lllll}10 & 272621 & 10\end{array}$ \\
\hline 13 & $\begin{array}{lllll}11 & 26 & 27 & 12 & 11\end{array}$ \\
\hline 14 & $\begin{array}{lllll}11 & 29 & 21 & 26 & 11\end{array}$ \\
\hline 15 & 22921192 \\
\hline 16 & $\begin{array}{lllll}13 & 28 & 18 & 20 & 13\end{array}$ \\
\hline 17 & $\begin{array}{lllll}2 & 29 & 24 & 19 & 2\end{array}$ \\
\hline 18 & $\begin{array}{lllll}17 & 26 & 21 & 19 & 17\end{array}$ \\
\hline 19 & $\begin{array}{lllll}18 & 4 & 25 & 28 & 18\end{array}$ \\
\hline 20 & $\begin{array}{lllll}19 & 24 & 29 & 21 & 19\end{array}$ \\
\hline 21 & $\begin{array}{llllll}5 & 6 & 1 & 10 & 22 & 5\end{array}$ \\
\hline 22 & $\begin{array}{llllll}5 & 25 & 4 & 10 & 22 & 5\end{array}$ \\
\hline 23 & $\begin{array}{llllll}5 & 29 & 21 & 10 & 22 & 5\end{array}$ \\
\hline 24 & $\begin{array}{llllll}0 & 16 & 3 & 9 & 8 & 0\end{array}$ \\
\hline 25 & $\begin{array}{llllll}5 & 29 & 11 & 12 & 25 & 5\end{array}$ \\
\hline 26 & 56192295 \\
\hline 27 & $\begin{array}{llllll}5 & 6 & 3 & 24 & 29 & 5\end{array}$ \\
\hline 28 & $\begin{array}{llllll}5 & 13 & 20 & 2 & 29 & 5\end{array}$ \\
\hline 29 & $\begin{array}{llllll}1 & 10 & 4 & 18 & 17 & 1\end{array}$ \\
\hline 30 & $\begin{array}{llllll}6 & 1 & 10 & 21 & 19 & 6\end{array}$ \\
\hline 31 & $\begin{array}{llllll}6 & 1 & 17 & 26 & 19 & 6\end{array}$ \\
\hline 32 & $\begin{array}{llllll}6 & 1 & 27 & 26 & 19 & 6\end{array}$ \\
\hline 33 & $\begin{array}{llllll}1 & 10 & 9 & 8 & 17 & 1\end{array}$ \\
\hline 34 & $\begin{array}{llllll}7 & 4 & 10 & 9 & 11 & 7\end{array}$ \\
\hline 35 & $\begin{array}{llllll}7 & 4 & 25 & 12 & 11 & 7\end{array}$ \\
\hline 36 & $\begin{array}{llllll}7 & 24 & 19 & 26 & 11 & 7\end{array}$ \\
\hline 37 & 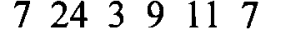 \\
\hline 38 & $\begin{array}{llllll}1 & 10 & 21 & 19 & 17 & 1\end{array}$ \\
\hline 39 & $\begin{array}{llllll}8 & 0 & 14 & 11 & 9 & 8\end{array}$ \\
\hline 40 & $\begin{array}{llllll}8 & 23 & 24 & 3 & 9 & 8\end{array}$ \\
\hline 41 & $\begin{array}{llllll}8 & 17 & 26 & 11 & 9 & 8\end{array}$ \\
\hline 42 & $\begin{array}{llllll}8 & 23 & 24 & 19 & 17 & 8\end{array}$ \\
\hline
\end{tabular}

Solution \#2

$\begin{array}{llll}0 & 1614 & 0\end{array}$

$\begin{array}{llll}127 & 10 & 1\end{array}$

$\begin{array}{llll}5 & 22 & 13 & 5\end{array}$

$\begin{array}{llll}13 & 20 & 15 & 13\end{array}$

$\begin{array}{llll}17 & 26 & 19 & 17\end{array}$

$\begin{array}{llll}19 & 26 \quad 21 & 19\end{array}$

$\begin{array}{lllll}1 & 27 & 26 & 17 & 1\end{array}$

$\begin{array}{lllll}5 & 13 & 28 & 25 & 5\end{array}$

$\begin{array}{lllll}6 & 1 & 17 & 19 & 6\end{array}$

$\begin{array}{lllll}6 & 324196\end{array}$

$\begin{array}{lllll}7 & 24 & 29 & 11 & 7\end{array}$

$\begin{array}{lllll}10 & 27 & 26 & 21 & 10\end{array}$

$\begin{array}{lllll}11 & 26 & 27 & 12 & 11\end{array}$

$\begin{array}{lllll}11 & 29 & 21 & 26 & 11\end{array}$

22921192

$\begin{array}{lllll}13 & 28 & 18 & 20 & 13\end{array}$

$\begin{array}{lllll}2 & 29 & 24 & 19 & 2\end{array}$

$\begin{array}{lllll}17 & 26 & 21 & 19 & 17\end{array}$

$\begin{array}{lllll}18 & 4 & 25 & 28 & 18\end{array}$

$\begin{array}{lllll}19 & 24 & 29 & 21 & 19\end{array}$

$\begin{array}{llllll}5 & 6 & 1 & 10 & 22 & 5\end{array}$

$\begin{array}{llllll}5 & 25 & 4 & 10 & 22 & 5\end{array}$

$\begin{array}{llllll}5 & 29 & 21 & 10 & 22 & 5\end{array}$

$\begin{array}{llllll}0 & 16 & 3 & 9 & 8 & 0\end{array}$

$\begin{array}{llllll}5 & 29 & 11 & 12 & 25 & 5\end{array}$

$\begin{array}{llllll}5 & 6 & 19 & 2 & 29 & 5\end{array}$

$\begin{array}{llllll}5 & 6 & 3 & 24 & 29 & 5\end{array}$

$\begin{array}{llllll}5 & 13 & 20 & 2 & 29 & 5\end{array}$

$\begin{array}{llllll}1 & 10 & 4 & 18 & 17 & 1\end{array}$

$\begin{array}{llllll}6 & 1 & 10 & 21 & 19 & 6\end{array}$

$\begin{array}{llllll}6 & 1 & 17 & 26 & 19 & 6\end{array}$

$\begin{array}{llllll}6 & 1 & 27 & 26 & 19 & 6\end{array}$

$\begin{array}{llllll}1 & 10 & 9 & 8 & 17 & 1\end{array}$

$\begin{array}{llllll}7 & 4 & 10 & 9 & 11 & 7\end{array}$

$\begin{array}{lllllll}7 & 4 & 25 & 12 & 11 & 7\end{array}$

$\begin{array}{llllll}7 & 24 & 19 & 26 & 11 & 7\end{array}$

$\begin{array}{llllll}7 & 24 & 3 & 9 & 11 & 7\end{array}$

$\begin{array}{llllll}1 & 10 & 21 & 19 & 17 & 1\end{array}$

$\begin{array}{llllll}8 & 0 & 14 & 11 & 9 & 8\end{array}$

$\begin{array}{llllll}8 & 23 & 24 & 3 & 9 & 8\end{array}$

$\begin{array}{lllllll}8 & 17 & 26 & 11 & 9 & 8\end{array}$

$\begin{array}{llllll}8 & 23 & 24 & 19 & 17 & 8\end{array}$

- 194 - 
$43 \quad 9361109$

$\begin{array}{lllllll}44 & 9 & 11 & 12 & 27 & 10 & 9\end{array}$

$\begin{array}{lllllll}45 & 9 & 11 & 26 & 21 & 10 & 9\end{array}$

$\begin{array}{llllllll}46 & 8 & 23 & 15 & 20 & 18 & 17 & 8\end{array}$
93611109

$\begin{array}{llllll}9 & 11 & 12 & 27 & 10 & 9\end{array}$

$\begin{array}{llllll}9 & 11 & 29 & 21 & 10 & 9\end{array}$

$\begin{array}{lllllll}8 & 23 & 15 & 20 & 18 & 17 & 8\end{array}$

\begin{tabular}{|c|c|}
\hline 1 & $\begin{array}{lllll}0 & 16 & 14 & 0\end{array}$ \\
\hline 2 & $\begin{array}{llll}1 & 27 & 10 & 1\end{array}$ \\
\hline 3 & 522135 \\
\hline 4 & $\begin{array}{llll}13 & 20 & 15 & 13\end{array}$ \\
\hline 5 & $\begin{array}{llll}17 & 26 & 19 & 17\end{array}$ \\
\hline 6 & $192621 \quad 19$ \\
\hline 7 & $\begin{array}{lllll}1 & 27 & 26 & 17 & 1\end{array}$ \\
\hline 8 & $\begin{array}{lllll}5 & 13 & 28 & 25 & 5\end{array}$ \\
\hline 9 & $\begin{array}{lllll}6 & 1 & 17 & 19 & 6\end{array}$ \\
\hline 10 & 6324196 \\
\hline 11 & $\begin{array}{lllll}724 & 29 & 11 & 7\end{array}$ \\
\hline 12 & $\begin{array}{lllll}10 & 27 & 26 & 21 & 10\end{array}$ \\
\hline 13 & $\begin{array}{lllll}11 & 26 & 27 & 12 & 11\end{array}$ \\
\hline 14 & $\begin{array}{lllll}1129 & 21 & 26 & 11\end{array}$ \\
\hline 15 & $22921 \quad 192$ \\
\hline 16 & $\begin{array}{lllll}13 & 28 & 18 & 20 & 13\end{array}$ \\
\hline 17 & $22924 \quad 192$ \\
\hline 18 & $\begin{array}{lllll}17 & 26 & 21 & 19 & 17\end{array}$ \\
\hline 19 & $\begin{array}{lllll}18 & 4 & 25 & 28 & 18\end{array}$ \\
\hline 20 & $\begin{array}{llll}19 & 2429 & 21 & 19\end{array}$ \\
\hline 21 & $\begin{array}{llllll}5 & 6 & 1 & 10 & 22 & 5\end{array}$ \\
\hline 22 & $\begin{array}{llllll}5 & 25 & 4 & 10 & 22 & 5\end{array}$ \\
\hline 23 & $\begin{array}{llllll}5 & 29 & 21 & 10 & 22 & 5\end{array}$ \\
\hline 24 & 0163980 \\
\hline 25 & $\begin{array}{llllll}5 & 29 & 11 & 12 & 25 & 5\end{array}$ \\
\hline 26 & 56192295 \\
\hline 27 & 56324295 \\
\hline 28 & $\begin{array}{llllll}5 & 13 & 20 & 2 & 29 & 5\end{array}$ \\
\hline 29 & $\begin{array}{llllll}1 & 10 & 4 & 18 & 17 & 1\end{array}$ \\
\hline 30 & $\begin{array}{llllll}6 & 1 & 10 & 21 & 19 & 6\end{array}$ \\
\hline 31 & $\begin{array}{llllll}6 & 1 & 17 & 26 & 19 & 6\end{array}$ \\
\hline 32 & $\begin{array}{llllll}6 & 1 & 27 & 26 & 19 & 6\end{array}$ \\
\hline 33 & $\begin{array}{llllll}1 & 10 & 9 & 8 & 17 & 1\end{array}$ \\
\hline 34 & $\begin{array}{llllll}7 & 4 & 10 & 9 & 11 & 7\end{array}$ \\
\hline 35 & $\begin{array}{llllll}7 & 4 & 25 & 12 & 11 & 7\end{array}$ \\
\hline 36 & $\begin{array}{llllll}7 & 24 & 19 & 26 & 11 & 7\end{array}$ \\
\hline 37 & 724399117 \\
\hline
\end{tabular}

$\begin{array}{llll}0 & 16 & 14 & 0\end{array}$

$\begin{array}{llll}1 & 27 & 10 & 1\end{array}$

$\begin{array}{llll}5 & 22 & 13 & 5\end{array}$

$\begin{array}{llll}13 & 20 & 15 & 13\end{array}$

$\begin{array}{llll}17 & 26 & 19 & 17\end{array}$

$\begin{array}{llll}19 & 26 & 21 & 19\end{array}$

$\begin{array}{lllll}1 & 27 & 26 & 17 & 1\end{array}$

$\begin{array}{lllll}5 & 13 & 28 & 25 & 5\end{array}$

$\begin{array}{lllll}6 & 1 & 17 & 19 & 6\end{array}$

$\begin{array}{lllll}6 & 324196\end{array}$

$\begin{array}{lllll}7 & 24 & 29 & 11 & 7\end{array}$

$\begin{array}{lllll}10 & 27 & 26 & 21 & 10\end{array}$

$\begin{array}{lllll}11 & 26 & 27 & 12 & 11\end{array}$

$\begin{array}{lllll}11 & 29 & 21 & 26 & 11\end{array}$

22921192

$\begin{array}{lllll}13 & 28 & 18 & 20 & 13\end{array}$

$\begin{array}{lllll}2 & 29 & 24 & 19 & 2\end{array}$

$\begin{array}{lllll}17 & 26 & 21 & 19 & 17\end{array}$

$\begin{array}{lllll}18 & 4 & 25 & 28 & 18\end{array}$

$\begin{array}{lllll}19 & 24 & 29 & 21 & 19\end{array}$

$\begin{array}{llllll}5 & 6 & 1 & 10 & 22 & 5\end{array}$

$\begin{array}{llllll}5 & 25 & 4 & 10 & 22 & 5\end{array}$

$\begin{array}{llllll}5 & 29 & 21 & 10 & 22 & 5\end{array}$

$\begin{array}{llllll}0 & 16 & 3 & 9 & 8 & 0\end{array}$

$\begin{array}{llllll}5 & 29 & 11 & 12 & 25 & 5\end{array}$

$\begin{array}{llllll}5 & 6 & 19 & 2 & 29 & 5\end{array}$

$\begin{array}{llllll}5 & 6 & 3 & 24 & 29 & 5\end{array}$

$\begin{array}{llllll}5 & 13 & 20 & 2 & 29 & 5\end{array}$

$\begin{array}{llllll}1 & 10 & 4 & 18 & 17 & 1\end{array}$

$\begin{array}{llllll}6 & 1 & 10 & 21 & 19 & 6\end{array}$

$\begin{array}{llllll}6 & 1 & 17 & 26 & 19 & 6\end{array}$

$\begin{array}{llllll}6 & 1 & 27 & 26 & 19 & 6\end{array}$

$\begin{array}{llllll}1 & 10 & 9 & 8 & 17 & 1\end{array}$

$\begin{array}{llllll}7 & 4 & 10 & 9 & 11 & 7\end{array}$

$\begin{array}{llllll}7 & 4 & 25 & 12 & 11 & 7\end{array}$

$\begin{array}{llllll}7 & 24 & 19 & 26 & 11 & 7\end{array}$

$\begin{array}{llllll}7 & 24 & 3 & 9 & 11 & 7\end{array}$ 
$\begin{array}{llllllll}38 & 1 & 10 & 21 & 19 & 17 & 1\end{array}$

$\begin{array}{lllllll}39 & 8 & 0 & 14 & 11 & 9 & 8\end{array}$

$\begin{array}{lllllll}40 & 8 & 23 & 24 & 3 & 9 & 8\end{array}$

$\begin{array}{lllllll}41 & 8 & 17 & 26 & 11 & 9 & 8\end{array}$

$\begin{array}{llllllll}42 & 8 & 23 & 24 & 19 & 17 & 8\end{array}$

$\begin{array}{llllllll}43 & 9 & 3 & 6 & 1 & 10 & 9\end{array}$

$\begin{array}{lllllll}44 & 9 & 11 & 12 & 27 & 10 & 9\end{array}$

$\begin{array}{lllllll}45 & 9 & 3 & 16 & 14 & 11 & 9\end{array}$

$\begin{array}{llllllll}46 & 8 & 23 & 15 & 20 & 18 & 17 & 8\end{array}$ $\begin{array}{llllll}1 & 10 & 21 & 19 & 17 & 1\end{array}$

$\begin{array}{lllllll}8 & 0 & 14 & 11 & 9 & 8\end{array}$

$\begin{array}{llllll}8 & 23 & 24 & 3 & 9 & 8\end{array}$

$\begin{array}{llllll}8 & 17 & 26 & 11 & 9 & 8\end{array}$

$\begin{array}{llllll}8 & 23 & 24 & 19 & 17 & 8\end{array}$

$\begin{array}{llllll}9 & 3 & 6 & 1 & 10 & 9\end{array}$

$\begin{array}{lllllll}9 & 11 & 12 & 27 & 10 & 9\end{array}$

$\begin{array}{llllll}10 & 21 & 19 & 26 & 27 & 10\end{array}$

$\begin{array}{lllllll}8 & 23 & 15 & 20 & 18 & 17 & 8\end{array}$

- 196 - 